\title{
DEPTH SENSITIVE VISION-BASED HUMAN-COMPUTER \\ INTERACTION USING NATURAL ARM/FINGER \\ GESTURES: AN EMPIRICAL INVESTIGATION
}

By

Farzin Farhadi-Niaki, B.Sc.

A thesis submitted to the Faculty of Graduate and Post Doctoral Affairs in partial fulfillment of the requirements for the degree of

Master of Applied Science

in

Electrical and Computer Engineering

Carleton University

Ottawa, Ontario

(C) 2011

Farzin Farhadi-Niaki 
Library and Archives

Canada

Published Heritage

Branch

395 Wellington Street

Ottawa ON K1A ON4

Canada
Bibliothèque et

Archives Canada

Direction du

Patrimoine de l'édition

395 , rue Wellington

Ottawa ON K1A ON4

Canada
Your file Votre référence

ISBN: 978-0-494-87814-9

Our file Notre référence

ISBN: 978-0-494-87814-9

\section{NOTICE:}

The author has granted a nonexclusive license allowing Library and Archives Canada to reproduce, publish, archive, preserve, conserve, communicate to the public by telecommunication or on the Internet, loan, distrbute and sell theses worldwide, for commercial or noncommercial purposes, in microform, paper, electronic and/or any other formats.

The author retains copyright ownership and moral rights in this thesis. Neither the thesis nor substantial extracts from it may be printed or otherwise reproduced without the author's permission.
AVIS:

L'auteur a accordé une licence non exclusive permettant à la Bibliothèque et Archives Canada de reproduire, publier, archiver, sauvegarder, conserver, transmettre au public par télécommunication ou par l'Internet, prêter, distribuer et vendre des thèses partout dans le monde, à des fins commerciales ou autres, sur support microforme, papier, électronique et/ou autres formats.

L'auteur conserve la propriété du droit d'auteur et des droits moraux qui protege cette thèse. $\mathrm{Ni}$ la thèse ni des extraits substantiels de celle-ci ne doivent être imprimés ou autrement reproduits sans son autorisation.
In compliance with the Canadian Privacy Act some supporting forms may have been removed from this thesis.

While these forms may be included in the document page count, their removal does not represent any loss of content from the thesis.
Conformément à la loi canadienne sur la protection de la vie privée, quelques formulaires secondaires ont été enlevés de cette thèse.

Bien que ces formulaires aient inclus dans la pagination, il n'y aura aucun contenu manquant. 
The undersigned hereby recommend to the Faculty of Graduate and Post Doctoral Affairs acceptance of the thesis

\section{DEPTH SENSITIVE VISION-BASED HUMAN-COMPUTER INTERACTION \\ USING NATURAL ARM/FINGER GESTURES: AN EMPIRICAL INVESTIGATION}

submitted by

\section{Farzin Farhadi-Niaki}

in partial fulfillment of the requirements for the degree of Master of Applied Science in Electrical and Computer Engineering

Chair, Howard Schwartz, Department of Systems and Computer Engineering

Thesis Supervisor, Ali Arya

Ottawa-Carleton Institute of Electrical and Computer Engineering (OCIECE)

Faculty of Engineering and Design

Department of Systems and Computer Engineering

Carleton University

December 2011 


\begin{abstract}
We present a novel user interface based on arm/hand gestures for interactive applications with small or large-scale displays. We have shown that combining mouse with arm and finger gestures provides more interesting and immersive ways to perform typical desktop operations using 3D data of the scenes.

We have developed a vision-based HCI prototype to be employed as the basis for our comprehensive usability study on the use of arm/hand gestures for interaction with computers. Using the Kinect depth camera and OpenNI we secured our system with high stability and efficiency by decreasing the ambient disturbing factors such as noise or light condition dependency. In our prototype, we designed a capable algorithm using NITE and OpenCV to recognize arm and finger gestures. Finally, through a comprehensive user experiment we compared our natural gestures (finger and arm) to each other and also to the conventional input devices (mouse/keyboard), for simple and complicated tasks, and in two different situations (small and big screen displays) for precision, efficiency, easeof-use, fun-to-use, fatigue, naturalness, and overall satisfaction to verify the following hypothesis: on a WIMP user interface, the gesture-based input is superior to mouse/keyboard when using big screen; and the finger-based gesture input is superior to arm-based in the long term of use. Our empirical investigation also proves that gestures are more natural and pleasant to be used than mouse/keyboard. However, arm gestures cause more fatigue than mouse. This drawback is diminished when using finger gestures for input.
\end{abstract}




\section{Acknowledgments}

First and foremost I would like to offer my sincerest gratitude to my supervisor Dr. Ali Arya for his vast knowledge and experience that contributed a lot to my academic growth, and his great attributes of an inspirational teacher, researcher, and human being.

Next, I would like to acknowledge my good friends and colleagues Reza GhassemAghaei and S. Ali Etemad for their great contributions in the usability part of this research, and also Colin Killby for his aid in designing our user interface.

Last but not least, my special thanks go to my dear parents and brothers who granted me their unlimited love, faith, and support without which I would not be where I am today.

Finally I dedicate this thesis to the dearest and the most valuable and significant person in my life, my son Arad, for his patience, encouragements and brilliant ideas that supported me during my Master's program. 


\section{Table of Contents}

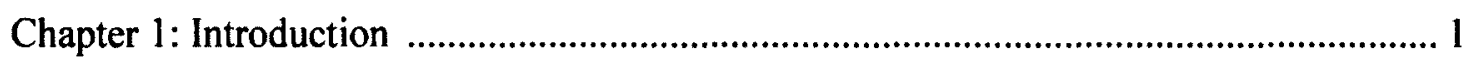

1.1. Human-Computer Interaction .............................................................................. 1

1.1.1. Gesture Recognition ................................................................................. 2

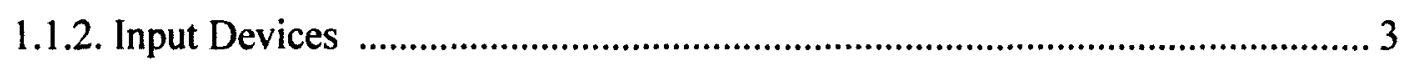

1.1.2.1. Microsoft Kinect Depth Camera .............................................................. 4

1.1.3. Natural Interaction, Graphics and Vision API's .............................................. 6

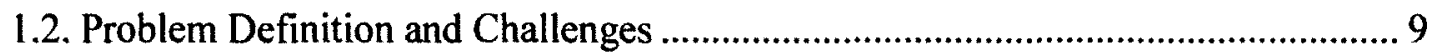

1.3. Research Objectives and Methodology ……...................................................... 10

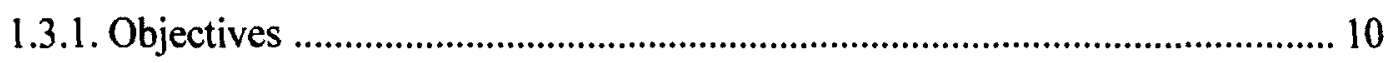

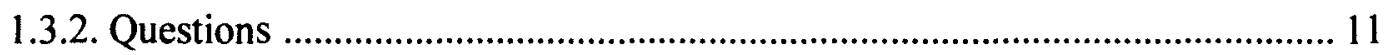

1.3.2.1. Hypothesis Discussion ....................................................................... 12

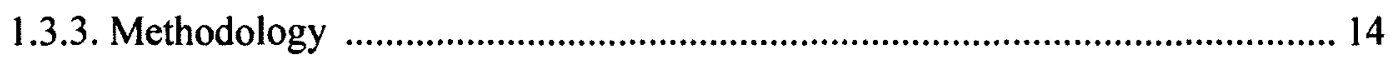

1.3.3.1. User Interface Design ............................................................................. 14

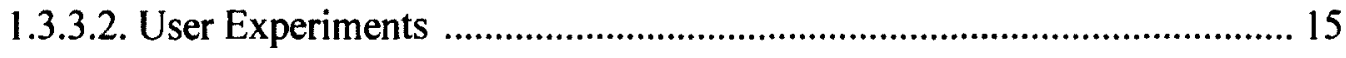

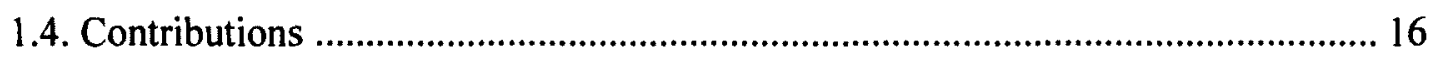

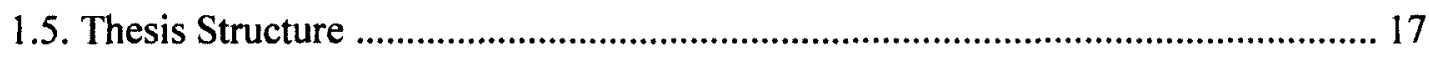

Chapter 2: Related Work ....................................................................................... 19

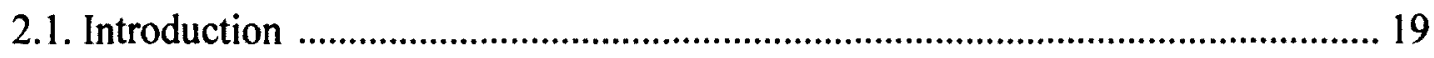

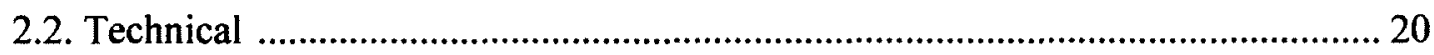

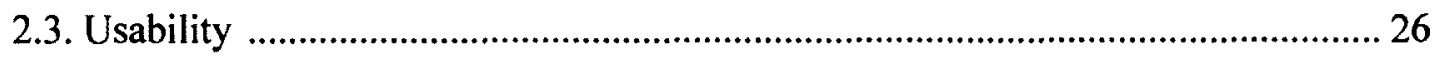

Chapter 3: Gesture Recognition ................................................................................ 30 


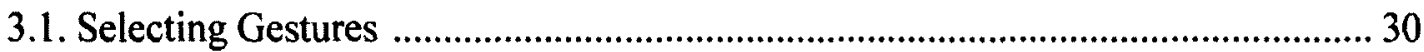

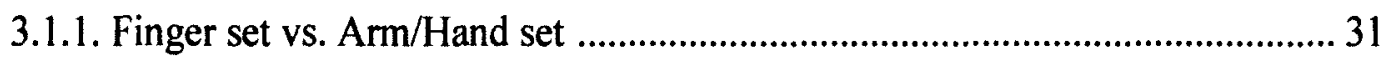

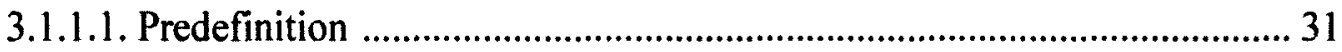

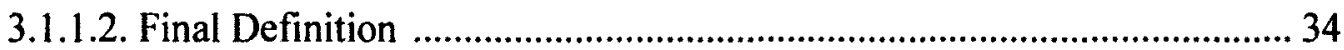

3.2. Fingertips Detection ...................................................................................... 35

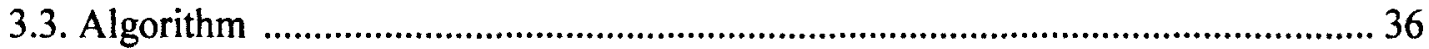

Chapter 4: UI and Experiment Design ........................................................................ 41

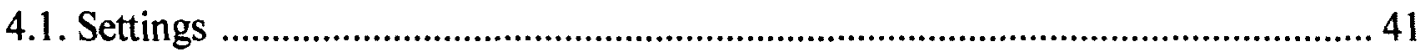

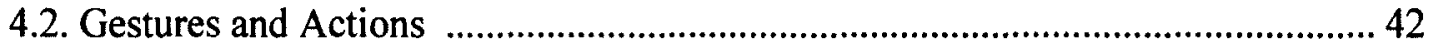

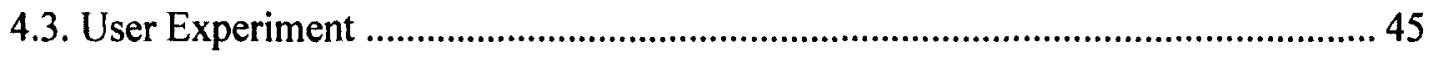

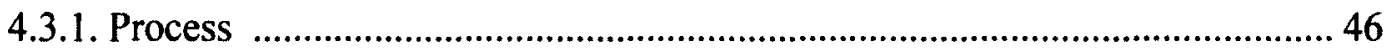

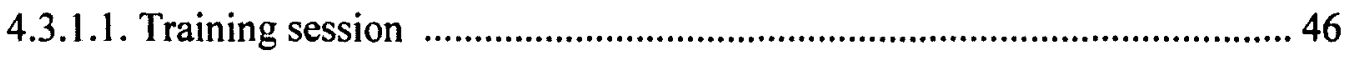

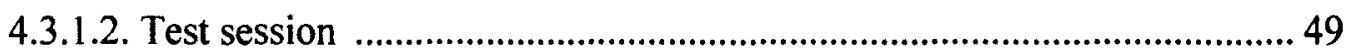

4.3.2. Questionnaire and Observation ................................................................... 54

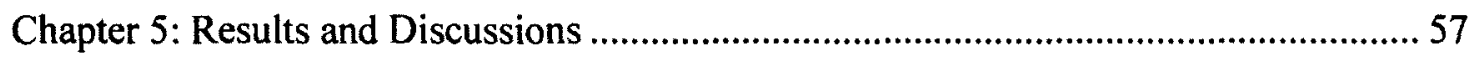

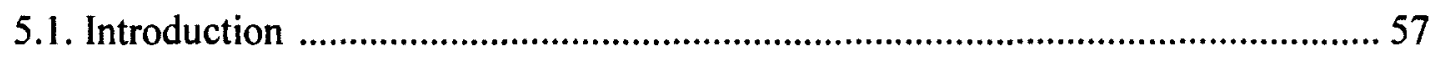

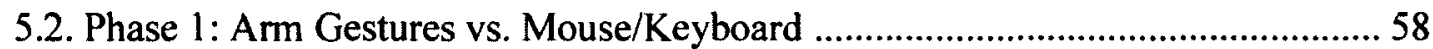

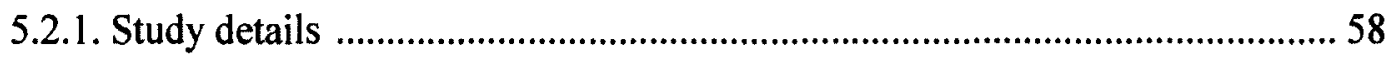

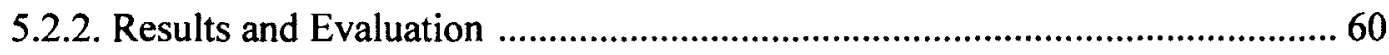

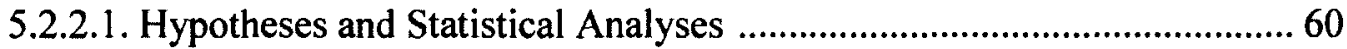

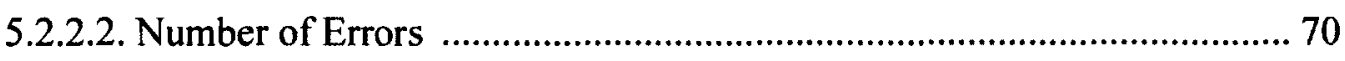

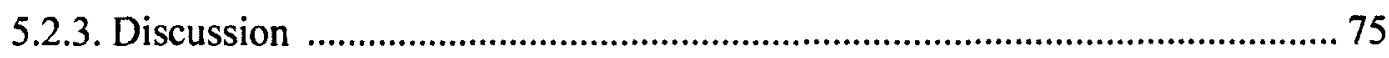

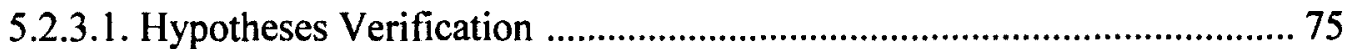




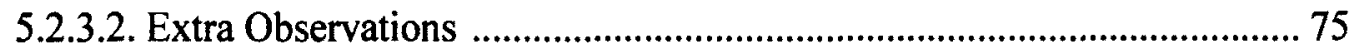

5.3. Phase 2: Finger Gestures vs. Arm Gestures ...................................................... 79

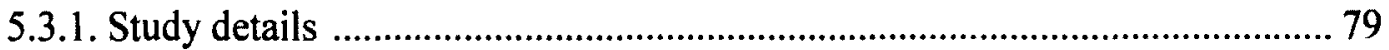

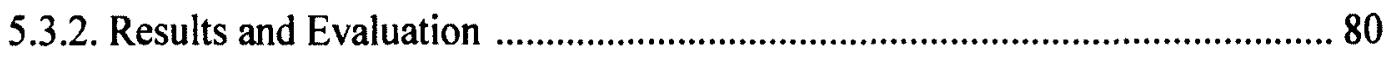

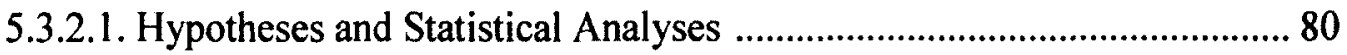

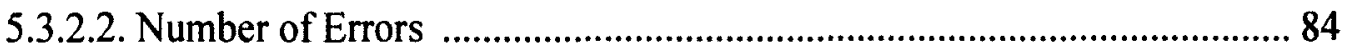

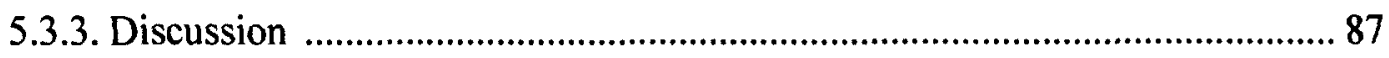

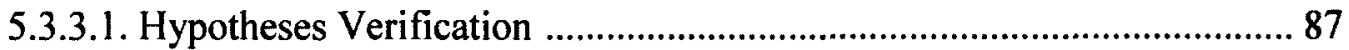

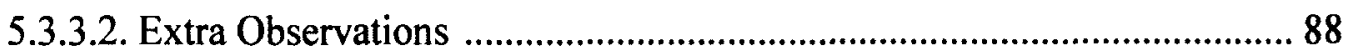

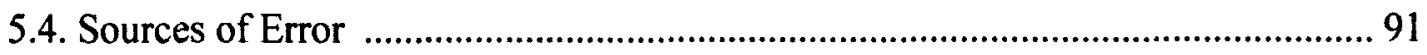

5.5. Users' Comments Summary ............................................................................. 91

Chapter 6: Conclusion ............................................................................................. 93

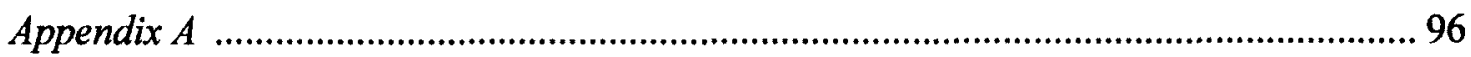

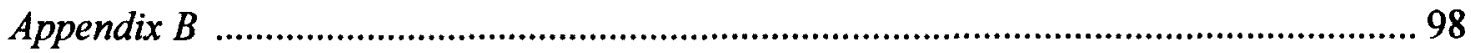

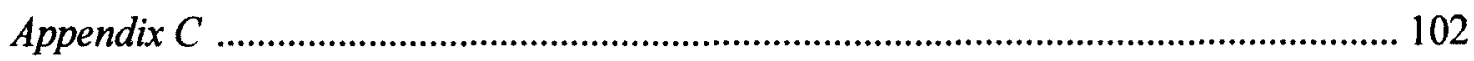

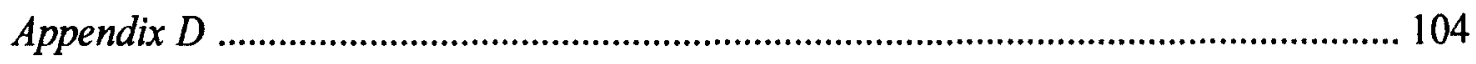

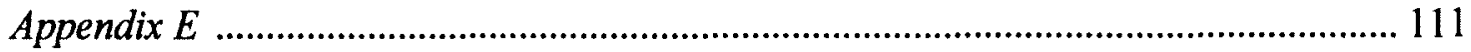

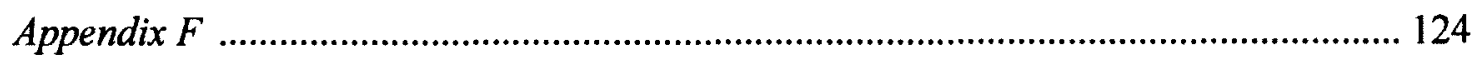

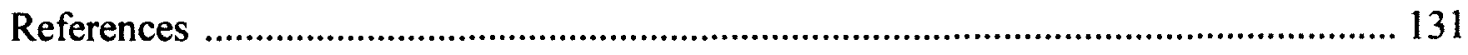




\section{List of Figures}

Figure 1.1. Kinect camera _....................................................................................................... 5

Figure 1.2. OpenNI: Abstract layer view [57] ...................................................................... 7

Figure 1.3. NITE Block Diagram [58] .......................................................................................... 8

Figure 1.4. WIMP user interface design ........................................................................................ 15

Figure 2.1. Three common phases employed by gesture recognition systems [36]......... 20

Figure 2.2. ArSLAT System Architecture [54] .................................................................... 22

Figure 3.1. Fingertips detection algorithm in OpenCV [61] .............................................. 35

Figure 3.2. Finger/Arm detection steps ...............................................................................3 37

Figure 3.3. Finger detection steps .................................................................................................. 38

Figure 3.4. The algorithm controlling UI using arm gestures recognition (similar to finger gestures with replacing Push and Circle to Tap and Pinch) ....................................... 39

Figure 3.5. NITE algorithm to detect arm gestures: (a) session state automation, (b) compound control [62] .................................................................................................................. 40

Figure 4.1. Finger tapping gesture ........................................................................................... 43

Figure 4.2. Finger pinching gesture ..................................................................................... 44

Figure 4.3. Training session ................................................................................................................. 49

Figure 4.4. UI: start session ................................................................................................ 50

Figure 4.5. UI: Pic.jpg is open ................................................................................................... 51

Figure 4.6. UI: Documents is open ........................................................................................ 52

Figure 4.7. UI: Pic2.jpg is open ................................................................................. 53

Figure 4.8. UI: Computer is open ......................................................................................... 53 
Figure 4.9. UI: Computer icon moves ................................................................................ 54

Figure 5.1. Interface …........................................................................................................... 59

Figure 5.2. A participant is interacting with the big screen using arm gesture ................. 59

Figure 5.3. A participant is interacting with the desktop using arm gesture ..................... 60

Figure 5.4. Temporal MAX/MIN/MEAN/ST DEV facts ...................................................... 61

Figure 5.5. Mean and SD of fatigue comparing 1- mouse/keyboard and 2- arm gesture using a) desktop for simple task, b) big-screen for simple task, c) desktop for complex task, and d) big-screen for complex task. The dots on the boxplots represent the outliers

Figure 5.6. Mean and SD of naturalness comparing 1- mouse/keyboard and 2- arm gesture using a) desktop for simple task, b) big-screen for simple task, c) desktop for complex task, and d) big-screen for complex task. The dots on the boxplots represent the outliers................................................................................................................................................ 68

Figure 5.7. Satisfaction comparison ................................................................................... 76

Figure 5.8. Best/Worst satisfactions .......................................................................................... 77

Figure 5.9. Four primitive tasks .............................................................................................. 78

Figure 5.10. Interface ……...................................................................................................................... 80

Figure 5.11. Temporal MAX/MIN/MEAN/ST DEV facts ...................................................... 81

Figure 5.12. Satisfaction comparison ………………................................................................ 88

Figure 5.13. Best/Worst satisfactions ........................................................................................... 89

Figure 5.14. Four primitive tasks ................................................................................................... 89

Figure 5.15. Gestures errors in simple task ............................................................................ 90

Figure 5.16. Gestures errors in complex task ............................................................................ 90 
Figure E.1. Perspective projection ......................................................................................... 112

Figure E.2. Image and scene planes ................................................................................................. 112

Figure E.3. Relationships between camera and global reference frames ......................... 116

Figure E.4. A trajectory is a virtual or mathematical encoding of a series of positions and orientations that an object visits over the time 120

Figure E.5. Active triangulation ............................................................................................. 122 


\section{List of Tables}

Table 1.1. A comparison between Microsoft and OpenNI SDKs [64] .................................. 8

Table 1.2. Mouse/keyboard vs. arm gesture .......................................................................... 12

Table 1.3. Arm gesture vs. finger gesture ................................................................................ 13

Table 3.1.a) Initial design for finger set vs. arm/hand set ...................................................... 32

Table 3.1.b) Initial design for finger set vs. arm/hand set (continued) ............................. 32

Table 3.2. Final design option for finger set vs. arm/hand set ........................................... 34

Table 4.1. (a) Arm and (b) Finger gestures' definitions, mouse analogies, and actions .42

Table 4.2. List of variables in our user experiment ................................................................ 45

Table 4.3. Evaluation criteria and their replying contexts (questions and measurements)

Table 4.4. Task table for Complex/Finger/Big-screen .......................................................... 55

Table 4.5. Questions for four primitive tasks, here for arm and finger (the same for mouse and arm) ............................................................................................................................... 55

Table 4.6. Observation for Complex/Arm/Desktop ............................................................... 56

Table 5.1. Task duration ................................................................................................................ 61

Table 5.2. Fatigue for simple task using desktop and results of t-test. ................................ 64

Table 5.3. Fatigue for simple task using big-screen and results of t-test ............................ 64

Table 5.4. Fatigue for complex task using desktop and results of t-test ........................... 64

Table 5.5. Fatigue for complex task using big-screen and results of t-test ........................ 64

Table 5.6. Naturalness for simple task using desktop and results of t-test ........................ 67

Table 5.7. Naturalness for simple task using big-screen and results of $t$-test .................... 67 
Table 5.8. Naturalness for complex task using desktop and results of t-test

Table 5.9. Naturalness for complex task using big-screen and results of t-test ...............67

Table 5.10. Observation for simple task using mouse on desktop .................................... 71

Table 5.11. Observation for simple task using mouse on big-screen ............................. 71

Table 5.12. Observation for simple task using gesture on desktop .................................. 72

Table 5.13. Observation for simple task using gesture on big-screen ............................... 72

Table 5.14. Observation for complex task using mouse on desktop .............................. 73

Table 5.15. Observation for complex task using mouse on big-screen ............................ 73

Table 5.16. Observation for complex task using gesture on desktop ............................. 74

Table 5.17. Observation for complex task using gesture on big-screen ............................ 74

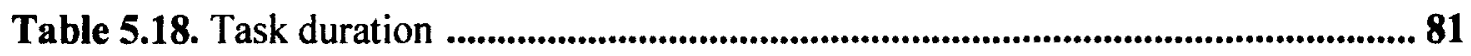

Table 5.19. Observation for simple task using finger on big-screen ................................ 85

Table 5.20. Observation for simple task using arm on big-screen .................................. 85

Table 5.21. Observation for complex task using finger on big-screen ................................86

Table 5.22. Observation for complex task using arm on big-screen .................................8 86

Table C.1. Table of results $\left({ }^{*} \mathrm{~T}=\right.$ Temporal resolution, ${ }^{* *} \mathrm{~S}=$ Spatial resolution)........ 102

Table C.2. Table of results $\left({ }^{*} \mathrm{~T}=\right.$ Temporal resolution, ${ }^{* *} \mathrm{~S}=$ Spatial resolution)........ 103

Table F.1. Questions for simple/mouse/desktop ................................................................ 124

Table F.2. Questions for simple/mouse/big-screen ................................................ 124

Table F.3. Questions for simple/gesture/desktop .................................................... 125

Table F.4. Questions for simple/gesture/big-screen ...................................................... 125

Table F.5. Questions for complex/mouse/desktop ................................................... 126

Table F.6. Questions for complex/mouse/big-screen .................................................. 126 
Table F.7. Questions for complex/gesture/desktop ................................................ 127

Table F.8. Questions for complex/gesture/big-screen ............................................... 127

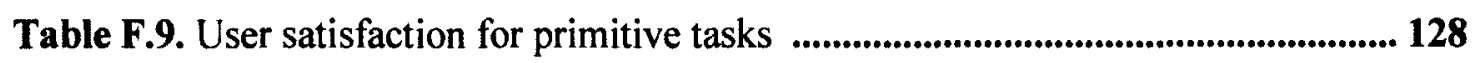

Table F.10. Questions for simple/finger/big-screen ..................................................... 128

Table F.11. Questions for simple/arm/big-screen ................................................ 129

Table F.12. Questions for complex/finger/big-screen .................................................... 129

Table F.13. Questions for complex/arm/big-screen ............................................ 130

Table F.14. User satisfaction for primitive tasks ........................................................ 130 


\section{Chapter 1: Introduction}

\subsection{Human-Computer Interaction}

Human-Computer Interaction (HCI) studies, plans, and designs the interaction between human and computing devices. $\mathrm{HCI}$ essentially aims to improve this interaction by making computers more practical and responsive to the user's requests, and in the long run its goal is to propose systems that minimize the difficulty between the human's cognitive model and the computer's ability to understand and respond properly. The relevant techniques on the machine side are implemented in operating systems, multimedia frameworks, development tools, and programming languages. The relevant topics on the human side, however include design disciplines, communication theory, social science, cognitive psychology, linguistics, and human factors e.g. user satisfaction [55]. 
Professional designers/researchers in $\mathrm{HCI}$ are generally concerned with the realistic application to real-world problems, and are involved in developing novel design methodologies, testing with innovative hardware devices, prototyping latest software systems, investigating new patterns for communication, and developing models and theories of interaction. New interaction technologies are among the most active areas of research in this regard.

\subsubsection{Gesture Recognition}

Lately the research in $\mathrm{HCI}$ is showing a significant focus on creating interfaces that are more user-friendly, by applying natural communication and human skills in the user interface design. The new wave of input systems in video game consoles (such as Nintendo Wii, Xbox Kinect, and PlayStation Move) are examples of the trend toward a more "natural" interfaces, where computers adapt to human behavior rather than the other way around. Ubiquitous Computing (also called Ambient or Pervasive Computing) is the extension of such trend where computing devices are integrated into "everyday" objects. Input/output techniques, interaction styles, and evaluation methods are mainly the challenging fields of research in gestural applications improvement [30].

Gesture recognition is an integral part of natural user interfaces used in order to interpret human gestures through mathematical algorithms. These gestures can be performed by different body parts (face or arm/hand in particular) to express human's emotion/posture, or interpret a sign language $[1,6]$. Machines can naturally interact and understand human body language/behaviors using gesture recognition and without the need to use mechanical devices like mouse and keyboard. For example if the user can control the 
screen pointer by pointing a finger, it could potentially make the conventional input devices such as mice, keyboards and even touch-screens redundant [7-12]. Computer vision and image processing techniques play the active roles in gesture recognition [2-5]. The scope of gesture recognition adoption includes, but is not limited to, the following: Immersive game technology: Providing immersive and interactive controls in game design [80].

Control through facial gestures: Controlling an application using facial gestures, and particularly gaze tracking for people with physical disabilities [8-12].

Virtual controllers: Offering a useful time saving controlling system e.g. in a television set or a car device [13].

Affective computing: Identifying emotional expression in a computer system [8-12].

Remote control: Remotely controlling various devices through a system [14-16].

\subsubsection{Input Devices}

A gesture recognition application should depend on its related input devices. With these devices the system can track the user's movements and eventually perform an action by recognizing the gestures. Employing a proper input device and environment in such a system demands suitable hardware and a proper Application Programming Interface (API) to provide software facilities.

Single regular camera is a conventional vision based input device to capture the image where is not necessarily as effective as depth-aware/stereo cameras while still is more demanding through simple applications [21]. 
Stereo cameras are a combination of two cameras represents a 3D data of the scene. Using a positioning reference such as infrared emitters provides the camera's relations [18].

Gesture-based controllers capture the motion of body parts in the area of interest, to be recognized and perform a linked task, e.g. the Wii Remote [19] [20].

While traditional computer vision has been mostly dependent on standard cameras, using depth-aware cameras, as a new generation of inexpensive 3D cameras, one can generate the scene's depth map to produce a 3D view for further processes, e.g. detection of hand gestures [17].

\subsubsection{Microsoft Kinect Depth Camera}

Microsoft Kinect (originally known as Project Natal) is a motion sensing input device for Xbox 360 video game console. It enables users to interact naturally through gestures and spoken commands with their games without a need of handheld controllers. Selling over 8 million units just in the first two months after its release in November 2010, caused a new record of "fastest selling consumer electronics device" in the Guinness World Record. Microsoft released its non-commercial SDK for Windows in June 16, 2011 which enables the developers to write their programs with $\mathrm{C}++, \mathrm{CH}$, and Visual Basic .NET. 


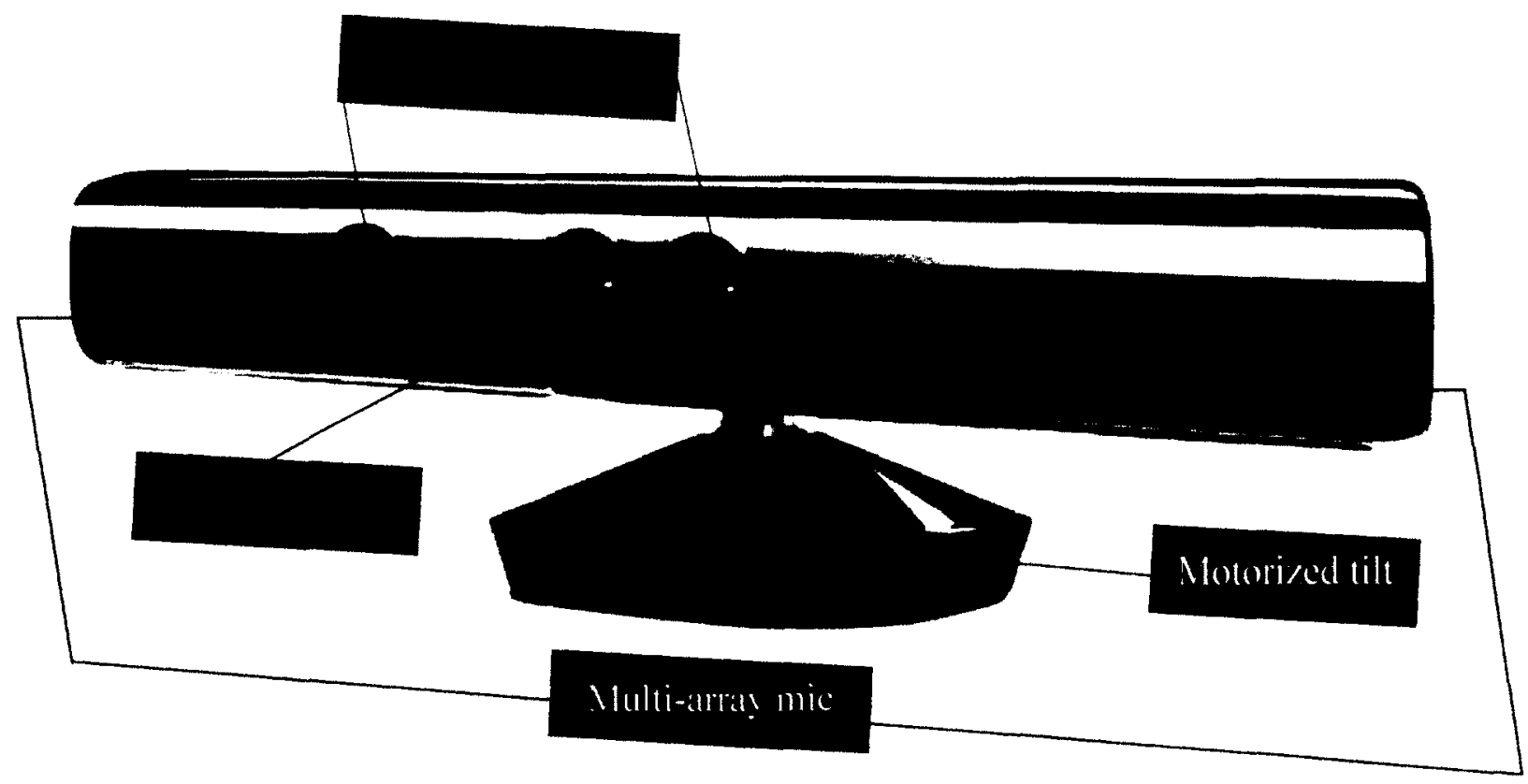

Figure 1.1. Kinect camera.

More details about the technology in Microsoft Kinect depth camera can be found in Appendix A.

\section{How does the Microsoft Kinect controller work?}

In spite of the fact that a Kinect unit is double price of two webcams, calibrating two single cameras are a bit harder, plus Kinect uses the more stable methods. Kinect uses some very useful techniques such as: background removal (no matter how busy the environment is), image segmentation and skeletonizing (a connected set of bones and joints at the bend-points), depth and connectivity detection (a possibility to find overlapping portions of the skeleton), automatic face detection using Haar filters, and hand gesture recognition.

Last but not least, Kinect also works well in an extensive variety of lighting conditions which itself helps in reducing the need for a high power of CPU. Having all these features enables Kinect to simulate a number of controllers properly [63]. 


\subsubsection{Natural Interaction, Graphics and Vision API's}

The concept of Natural Interaction (NI) addresses to the human-based type of HCI, mainly on vision and hearing senses. Some examples of human and machine natural interaction are such as: speech and command recognition to instruct devices, pre-defined hand gesture recognition to control the home electronic units, and body motion tracking to interact with a computer game.

\section{OpenCV}

OpenCV (Open source Computer Vision) is a library of programming functions for real time multidisciplinary computer vision. It has $\mathrm{C}, \mathrm{C}++$, and Python interfaces running on Windows, Linux, Android and Mac, with over 2500 optimized algorithms. The OpenCV library includes a variety of algorithms and provides many applications: contours, image parts and segmentation, histogram and matching, projection and 3D vision, tracking and motion (background subtraction, corner finding, optical flow, motion templates), camera calibration (functional to map the depth and RGB outputs of Kinect), structure from motion, SURF, face detection and Haar classifier.

\section{OpenNI}

OpenNI (Open Natural Interaction) designed by PrimeSense - the co-creator of Kinect [79] - is an open source, multi-language, and cross-platform framework that classifies APIs using natural interaction, in application development processes. OpenNI structures a standard API that can communicate with both vision/audio sensors (e.g. depth sensor of 
Kinect) and vision/audio perception middleware (e.g., NITE gesture analyzing software) independently. This standard API allows developers to write their natural interaction based applications regardless of middleware/sensor providers, and manipulate the 3D scenes of real life using the data collected from various sensors. A three-layered view of the OpenNI concept is shown in Figure 1.2.

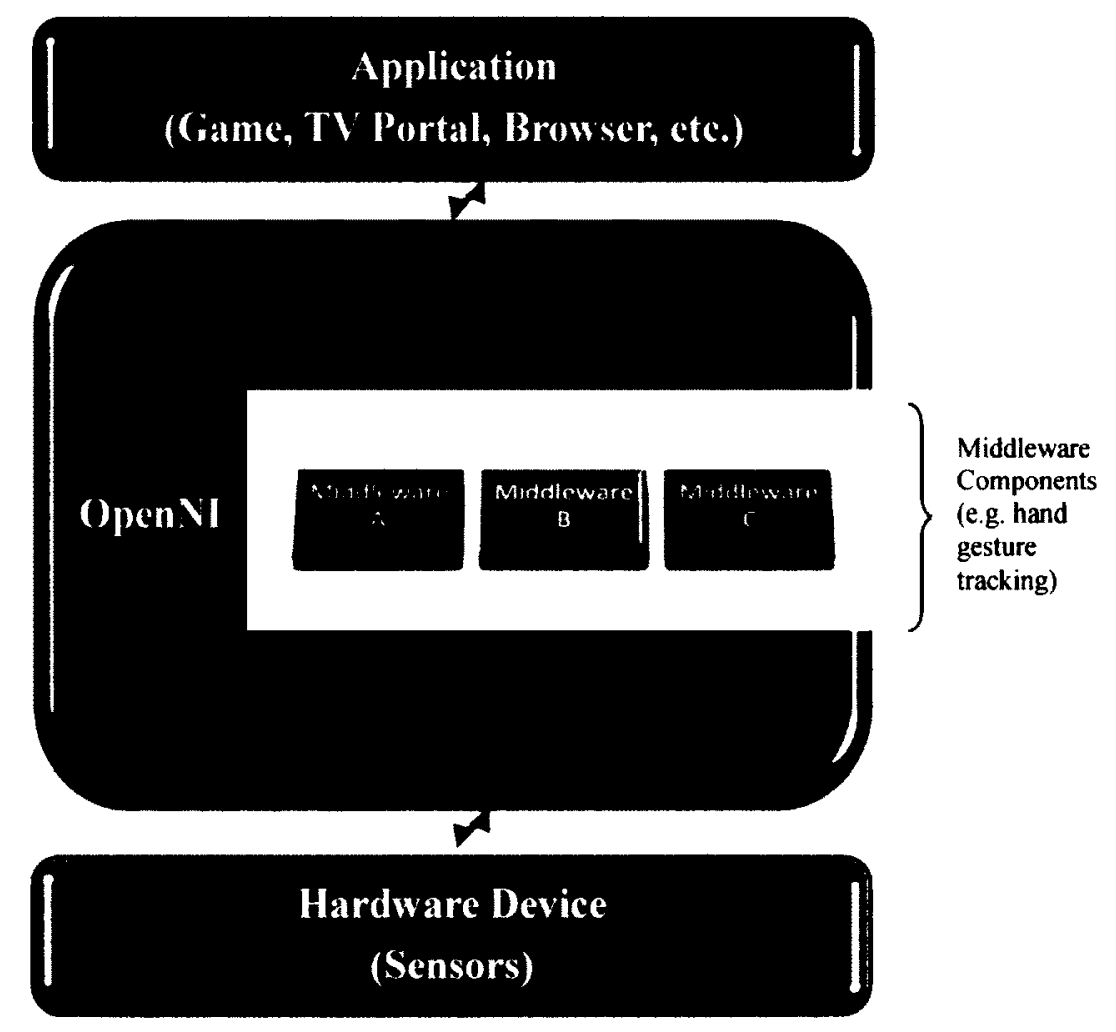

Figure 1.2. OpenNI: Abstract layer view (re-created based on [57]).

\section{Microsoft Kinect SDK vs. PrimeSense OpenNI}

According to the studies from Appendix B, Table 1.1 summarizes a comparison between Microsoft Kinect SDK and PrimeSense OpenNI SDK: 
Table 1.1. A comparison between Microsoft and OpenNI SDK's [64].

\begin{tabular}{|ll|}
\hline Mierosuft seems to work better & OpenNI seems to work better \\
\hline - with skeletons and/or audio & - when working on color point-clouds \\
- & on non-Windows 7 platforms \\
- & for commercial projects \\
- & when the sensor only sees the upper- \\
& body/hands \\
- & when there is a preference of an \\
existing framework to start with
\end{tabular}

\section{NITE}

NITE is a closed source toolbox that enables applications to translate the user's hand movement in traceable gestures (i.e. circle, push, swipe, etc.). Having additional interfaces located on top of OpenNI, NITE provides higher level results such as tracking a hand-point/skeleton, and analysing the scene.

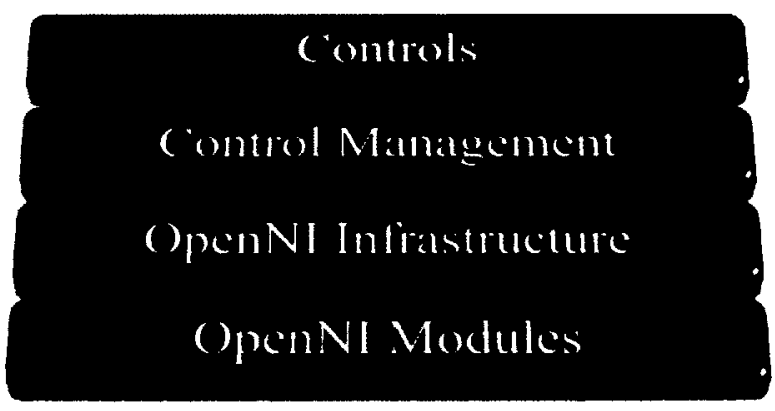

Figure 1.3. NITE Block Diagram (re-created based on [58]). 


\title{
OpenGL
}

OpenGL (Open Graphics Library) is a cross-language, cross-platform API for writing 2D and 3D graphic applications. The interface consists of over 250 different function calls which can be used to draw complex 3D scenes from simple primitives.

\begin{abstract}
Allegro
Allegro is a cross-platform library that mainly aims at multimedia programming, and handles common, low-level tasks such as accepting user input, creating windows, loading data, drawing images, playing sounds, etc.
\end{abstract}

\subsection{Problem Definition and Challenges}

Traditional $\mathrm{HCl}$ using mouse/keyboard presents a narrow variety of actions to the user, while its interaction metaphor is not easy to apply in smaller devices. Moreover, in some $\mathrm{HCI}$ applications, communication between human and machine using conventional controllers becomes cumbersome and unsuitable, whereas employing direct sensing and understanding of human hand gesture is a capable natural $\mathrm{HCI}$ tool. Vision-based studies, hand modeling, tracking, and gesture recognition are highlighted in this recent input modality [59].

On the other hand, accuracy and usefulness of gesture recognition software have remained a challenging issue. Noise, inconsistent lighting, items in the background, distinct features, and equipment limitations can be named as the constraints associated with image-based gesture recognition. 
Technological incompatibility may also cause difficulties in the general usage to match various image-based gesture recognition systems. For instance, a calibrating algorithm for one camera might not work properly for another different camera.

To achieve a required accuracy in outcome of some gesture recognition systems (i.e. hand tracking, hand posture recognition, gaze tracking, facial expressions, or head movements capturing), employing also robust computer vision methods are highly needed [22-30].

Finally, a consolidated and reliable usability analysis is essentially required to improve the ongoing research in $\mathrm{HCl}$, particularly for gesture-based input. Such a study has not been fulfilled as it deserves yet to shed light on designing a practical interaction between human and machines, and determining the application domains where gesture-based input is more suitable.

\subsection{Research Objectives and Methodology}

\subsubsection{Objectives}

\section{Phase 1:}

- To develop proper algorithms to detect arm gestures using the Kinect sensor and existing API's

- To compare two input methods (mouse-based and gesture-based inputs) in two different situations (small and big screen displays) for precision, efficiency, easeof-use, fun-to-use, fatigue, naturalness, and overall satisfaction to verify the following hypothesis:

For usability, and on a WIMP UI, the gesture-based input is superior to mouse/keyboard when using big screen. 


\section{Phase 2:}

- To develop proper algorithms to detect finger gestures using the Kinect sensor and existing API's

- To compare two input methods (arm-based and finger-based gestures) in two different situations (simple and complex tasks) for precision, efficiency, ease-ofuse, fun-to-use, fatigue, naturalness, and overall satisfaction to verify the following hypothesis:

For usability, and on a WIMP UI, the finger-based gesture input is superior to arm-based in the long term of use.

\subsubsection{Questions}

To design our $\mathrm{HCl}$ system we need to answer some questions such as:

1. What desktop actions do we want to control?

2. What gestures do we need to detect?

3. Should we use OpenCV library? Does it add much value in our case?

4. Can we add some new functionality with Kinect to OpenCV?

5. What hypotheses should be studied to compare the mouse/keyboard traditional inputs to the arm gestural inputs, and the arm-based gestures to the finger-based gestures inputs?

We have answered these questions in the following sections and chapter 5 . 


\subsubsection{Hypothesis Discussion}

Table 1.2 and 1.3 compares advantages and disadvantages of the three types of input in two different phases (mouse vs. arm gesture, and arm gesture vs. finger gesture) in a preassumption way. We expected to acquire more experimental facts during the technical process and also our hypothetical study.

Table 1.2. Mouse/keyboard vs. arm gesture.

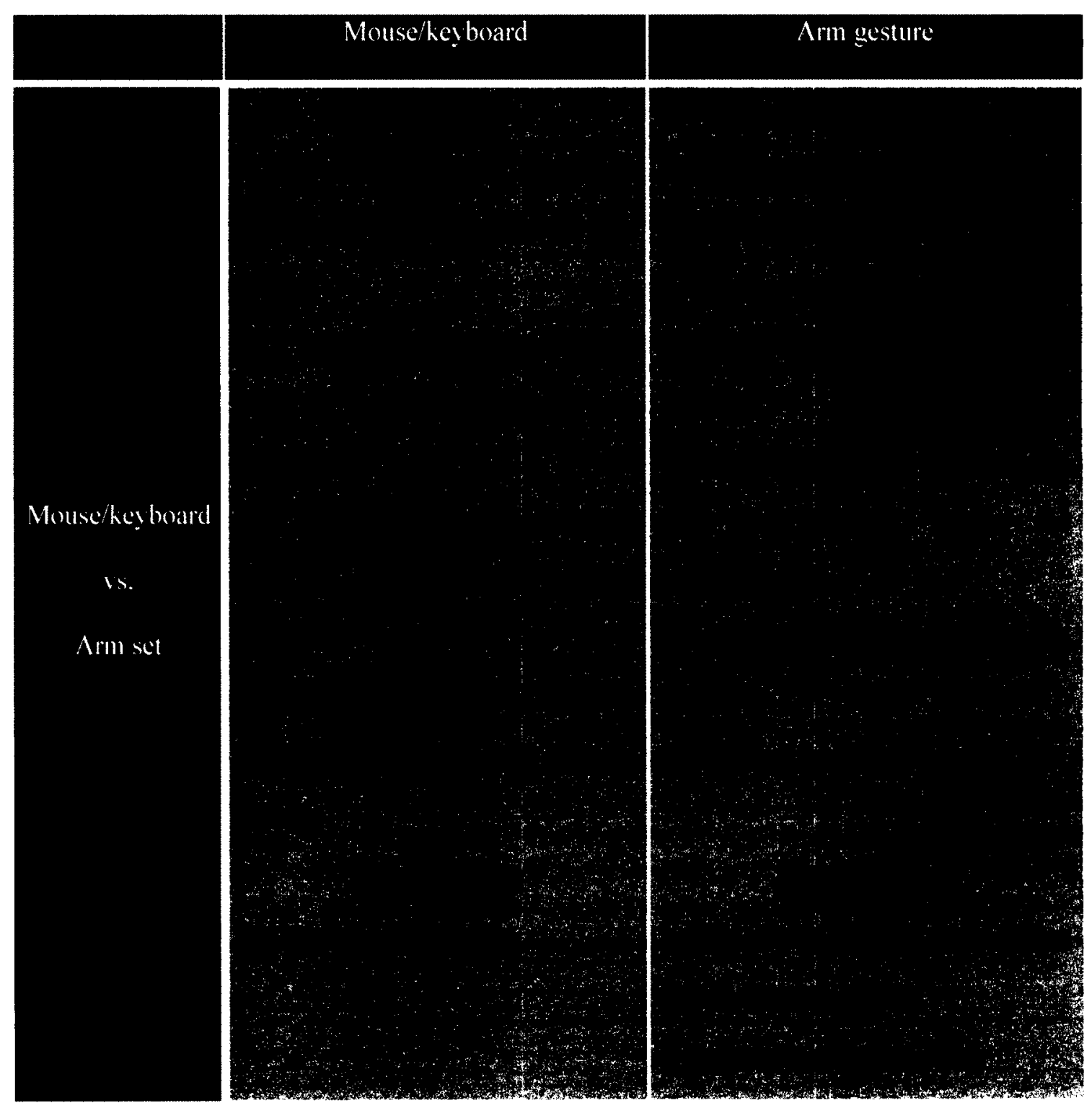


Table 1.3. Arm gesture vs. finger gesture.

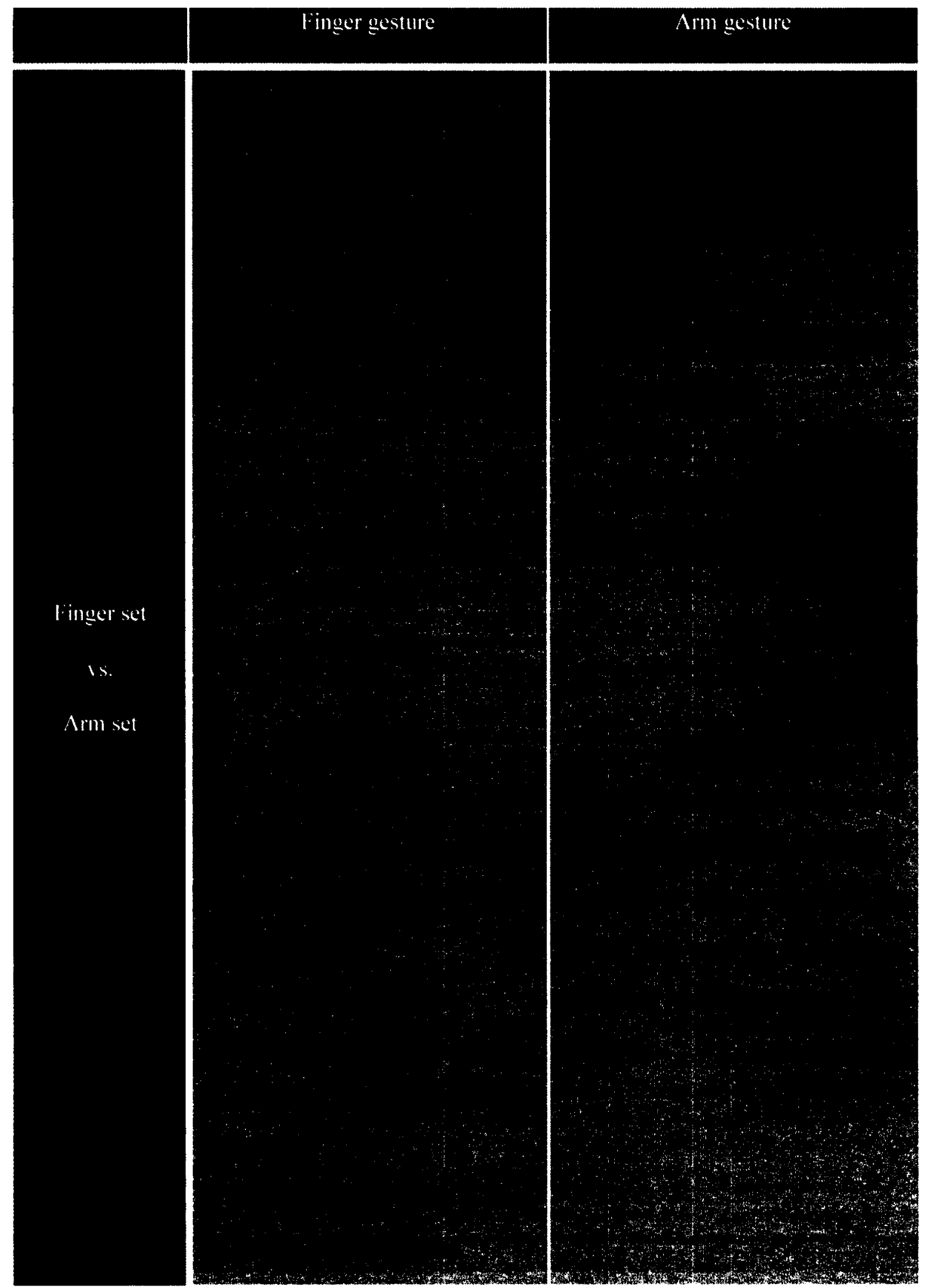




\subsubsection{Methodology}

\subsubsection{User Interface Design}

The point of communication between the user and the machine describes the humancomputer interface. This project uses a simulated WIMP interface which includes the following main parts in its user interface design:

- W: Windows

- I: Icons

- M: Menus

- P: Pointers

Novice users can learn WIMP user interfaces easily, as they are very good at abstracting workplaces due to their analogous paradigm to documents like paper sheets or folders. Having a rectangular region on a $2 \mathrm{D}$ flat screen makes them preferable to system developers while their generality also makes them a good fit in multitasking environments [81]. 


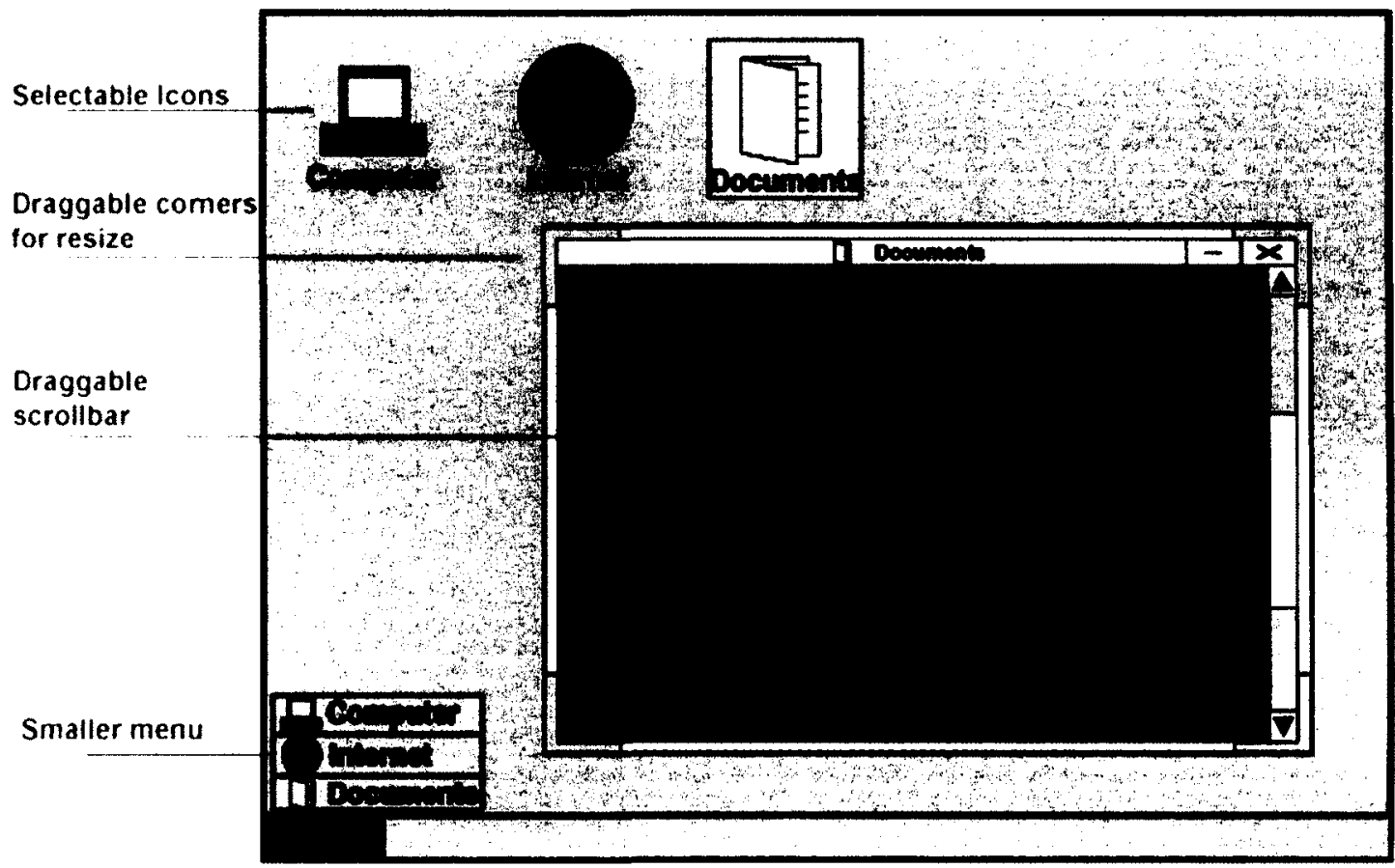

Figure 1.4. WIMP user interface design.

In a typical WIMP interface, as shown in Figure 1.4, upon opening an icon, a window appears as pictured, which the user can then resize, scroll, or close. A smaller menu can be good for a test of input control accuracy.

The design is kept as simple and minimalistic as possible, with neutral colors to reduce user error or bias.

We have used a combination of Kinect sensor, OpenCV, Allegro, OpenNI, and NITE to create a simulated desktop interface and interact with users.

\subsubsection{User Experiments}

In our usability experiments we have focused on common desktop tasks to be relatively general, and have included ratings by typical university users and also objective measures by observation, such as number of trials, errors, etc. 


\subsection{Contributions}

- Choice of natural gestures:

We first studied the possible natural gestures suitable for a WIMP user interface, and then defined the best matches of the predefined gestures to our prototype.

- Usability study for gesture-based input:

Through a comprehensive and hypothetical user experiment we have studied significant factors and usability in human-computer interaction concept, with comparing our natural defined gestures to each other and also to the conventional input devices (e.g. mouse/keyboard), along with evaluating different settings of desktop and big-screen, which can be a good source for further research in the field of NHCI. Our empirical investigation proves that gestures are more natural and pleasant to be used on big-screen displays than using mouse/keyboard. However, arm gestures cause more fatigue than mouse. This drawback is diminished when the gestural inputs are finger-based.

- System design (UI and gesture recognition) and relatively novel use of API's:

Using Kinect unit (as a commonly used vision-based input device) enables us to identify the depth of every single pixel in the frame by projecting a pattern of dots with the almost infrared laser over the scene, and establishing the parallax shift of the dot pattern for each pixel in the detector. In addition, using OpenNI and NITE has helped us to secure our system with a higher stability and efficiency, and to develop a capable algorithm to recognize the arm and finger gestures. 
Using the above explained method on our simulated desktop interface we can conserve the developing (no need for making samples and efforts in training, and testing sessions) and running time for gesture recognition and user interaction comparing to learning-based traditional method.

- The result of this work has been presented in Toronto Digifest 2011 as an invited guest speaker [77].

\subsection{Thesis Structure}

In the course of this text, the complete process of construction of the system explained earlier will be discussed.

In Chapter 2 a review of some key literature in the field of gestural $\mathrm{HCI}$, including technical and usability studies, is carried out.

Chapter 3 deals with the gesture recognition and its data types proposed in this research. Predefinition and the final selection process of the proposed gestures are discussed in this chapter as well. The last part of this chapter provides the algorithms we designed/developed to control our user interface objects by recognizing the arm and finger gestures.

Chapter 4 addresses more detail of the components engaged with our UI and experiment design. It reviews the hardware settings, our gestures and their relative actions. The experiment process and our evaluation method including the questionnaire and the observation are discussed in this chapter as well to facilitate our hypothetical studies on which the following chapter exploits the experimental results. 
Finally in Chapters 5 the experimental results are discussed and analyzed. This chapter is divided to two major phases:

- Phase 1- Arm gestures vs. Mouse/keyboard

- Phase 2- Finger gestures vs. Arm gestures

This chapter elaborately discusses the results, and analyzes them to verify our hypothetical objectives.

In Chapter 6 the concluding remarks and the potential areas and problems for future work are presented. This is followed by an overview on participants' comments and other supplementary documents which were utilized for this research, in appendixes. 


\section{Chapter 2: Related Work}

\subsection{Introduction}

The first hand gesture detectors that were developed used mechanical devices to capture information from a hand gesture [33]. One example of this early technology includes data glove devices, which collected the information generated from the movement of the fingers and transmitted it to a computer system [34] [35]. Over the past ten years, the performance of computer hardware has become significantly enhanced while units have steadily decreased in price. This improvement in technology has resulted in the gradual replacement of data glove devices by vision-based hand gesture technology. Vision-based technology does not require users to wear a device, making their gestures more natural because there are no limitations in the movements of the hand. It is also very userfriendly, which is essential in any human-computer interaction. Given that vision is one of the six physical media, vision-based technology is more desirable than wearable devices, such as the data glove device, in hand gesture recognition systems [31]. 


\subsection{Technical}

Recent studies have demonstrated that hand gesture systems are not only technical and theoretical in nature but are also very practical since they can be implemented into numerous types of application systems and environments. For example, Ahn et al. [46] developed a method for virtual environment slide show presentations.

Another example is the study by Jain [47], which describes a way to estimate hand poses for mobile phones that only have one pointing gesture based on a vision-based hand gesture approach. The sign language tutoring tool developed by Aran et al. [48] is also very practical because it is designed to interact with users to teach them the fundamentals of sign language [52].

As illustrated in Figure 2.1, hand gesture recognition systems are commonly divided into three phases including image pre-processing, tracking and recognition. Some theoretical background can also be found in Appendix F.

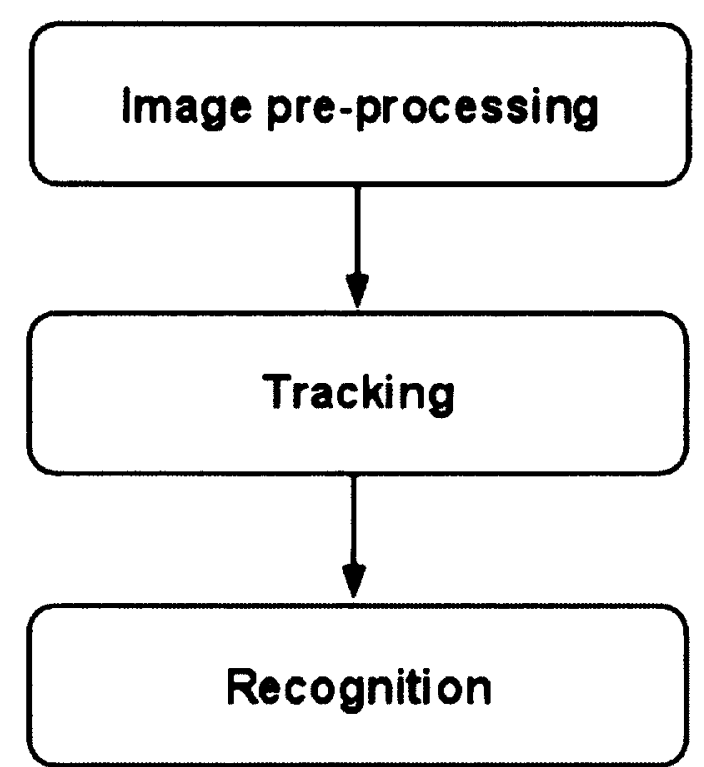

Figure 2.1. Three common phases employed by gesture recognition systems [36]. 
Several researchers have conducted similar studies in tracking, such as the Viola-Jonesbased cascade classifier, which is typically used for face tracking in rapid image processing [37] [38] and is regarded as more robust in pattern recognition against noise and lighting conditions [39]. Other researchers have shown that cascade classifiers can also be utilized to recognize hands and various parts of the human body [39-43].

In order to detect gestures, Marcel et al. [44] proposed a method of hand gesture recognition based on Input-Output Hidden Markov Models that track variations in the skin color of the human body. Similarly, Chen et al. [45] applied the hidden Markov model in training method to enable systems to detect hand postures, even though it is more complex than Cascade classifiers in training hand gestures.

In another study, Liu et al. [50] described a hand gesture recognition system aimed at enhanced Human-Computer Interaction. The AdaBoost algorithm was revised and used to automatically recognize a user's hand from the video stream, which is based on Haarlike features as a representation of hand gestures. A Multi-class Support Vector Machine was employed to train and detect the hand gesture based on $\mathrm{Hu}$ invariant moments features and the Human Computer Conversation was then implemented for hand gesture interaction instead of a traditional mouse and keyboard. A simple Human-Computer Interactive system that could detect predefined hand gestures for the numbers 0 to 6 was proposed by Liu et al. This system could better implement the Number Input Management in Word documents.

In order to translate the hand gestures, El-Bendary et al. [54] have studied on an automatic translation system of gestures for the alphabet that is used in the Arabic sign language. Their proposed Arabic Sign Language Alphabets Translator (ArSLAT) system 
does not rely on glove devices or visual markings. It uses images of bare hands, allowing the user to interact with the system in a natural manner. The ArSLAT system, as shown in Figure 2.2, employs five main phases. Their results indicate that the proposed ArSLAT system could detect the 30 hand gestures of the Arabic alphabet with an accuracy of $91.3 \%$.

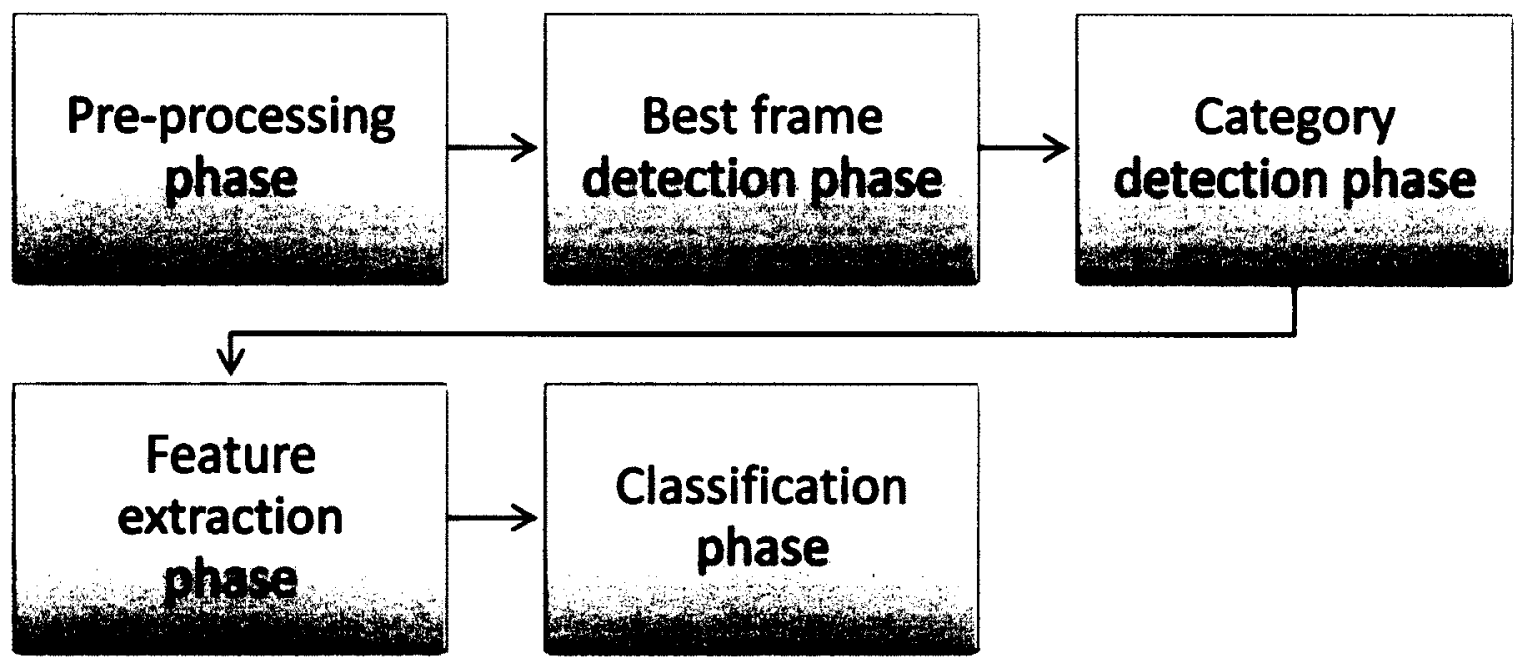

Figure 2.2. ArSLAT System Architecture [54].

The other research, by Yu et al. [53], proposes a hand gesture feature extraction method that employs multi-layer perception. Their studies demonstrate that two of the five common color spaces ( i.e., RGB, HSI, HSL, YCbCr, and YUV) for object segmentation, $\mathrm{YCbCr}$ and $\mathrm{HSI}$, are more appropriate for hand gesture image recognition and segmentation than the RGB color space. Hand color in the $\mathrm{YCbCr}$ color space is utilized to detect hand gestures. By binarizing the image and enhancing the contrast, the silhouette and distinct features of the hand are accurately and efficiently extracted from the image. Merging median and smoothing filters is their proposed approach to reduce background noise since the median filter removes the impulsive noise from the image and 
preserves sharp edges and the smoothing filter can reduce neighborhood radius to preserve the quality of the image. The Gauss-Laplace edge detection approach has been utilized to get the hand edge. A feature vector that can recognize hand gestures is developed from combinational parameters of $\mathrm{Hu}$ invariant moment, hand gesture region and Fourier descriptor. Their results demonstrate that the detection system (with a dataset of 3500 images) is significantly robust, as $97.4 \%$ of the hand gestures were accurately recognized.

On the other hand, Raheja et al. [51] have used Principal Component Analysis (PCA) method in their pattern matching. The PCA method is used because it is: (i) suitable for pattern matching since the human hand is used for gesture expression and its features (e.g. fingers, palm, and fist) are large enough compared to the background noise, and (ii) very fast compared to the neural network method, which necessitates high computation power and requires more time due to database training [51].

In above mentioned related works, accuracy and usefulness of gesture recognition software have remained a challenging issue. Noise, inconsistent lighting, items in the background, distinct features, and equipment limitations can also be named as the constraints associated with some of those image-based gesture recognition systems. Technological incompatibility may also cause difficulties in the general usage to match various image-based gesture recognition systems. For instance, a calibrating algorithm for one camera might not work properly for another different camera. In our gesture recognition prototype, however we have processed the $3 \mathrm{D}$ coordinates and RGB data provided by a Microsoft Kinect unit. The Kinect uses some more stable methods and very 
useful techniques such as: background removal, image segmentation, depth and connectivity detection, and hand gesture recognition. Last but not least, Kinect also works well in an extensive variety of lighting conditions which itself helps in reducing the need for a high power of CPU. Having all these features enables Kinect to simulate a number of controllers properly. Using Kinect unit enables us to identify the depth of every single pixel in the frame and ultimately conserve the developing (no need for making samples and efforts in training, and testing sessions) and running time comparing to the learningbased traditional methods that have been used in the above mentioned related works. Moreover, we have applied a depth thresholding, which removes the wrist and its unwanted defects from the depth map, based on $Z$ (creates a binary image). Cropping the wrist out of the frame can also help in improving accuracy. In terms of natural gestures selection, we have also studied all possible natural gestures, and then selected the best matches of our predefined gestures to our prototype. Using OpenNI and NITE we have secured our system with a high stability and efficiency by decreasing the effect of ambient disturbing factors such as noise and improper light conditions. In addition, programming with NITE provides some gesture detector options, e.g. Velocity or Angle features in a push detector in order to make a desirable setting for the push gesture recognition.

The objective of research in hand gesture recognition is to develop ways in which the human's hand can be utilized as an interface for human-computer interaction $(\mathrm{HCl})$. The shape, position and/or movement of the hand are parameters that are analyzed by visionbased hand gesture recognition systems. Specifically, hand gestures can be described by 
four main characteristics including hand configuration, palm orientation, hand position and hand movement. The flex angles of the fingers and the orientation of the palm are used to model static hand gestures whereas hand trajectories and orientation are also needed to model dynamic hand gestures. Therefore, to accurately model dynamic hand gestures, it is critical that the interpretation of dynamic gestures based on hand movement, shape and position is appropriate [49].

A series of hand gestures that in their entirety bear some meaning are defined as continuous gestures. The first step towards recognition includes the separation of a continuous gesture sequence into its component gestures, which is a complicated process because of "co-articulation". Co-articulation is a phenomenon by which one hand gesture influences the hand gesture that is next in a temporal sequence and is a very significant issue in recognizing hand gestures in fluent sign language. In an attempt by Bhuyan et al. [49] to resolve this issue, key frames in a sequence of gestures were selected and/or associated motion features were used during trajectory guided recognition. They examined how co-articulation can be detected and omitted from their proposed keyframe-based gesture recognition process. They proposed an acceleration feature that detects co-articulated hand gestures from other significant hand positions during trajectory-guided recognition of hand gestures since co-articulation involves fast hand movements compared to slower gestures.

Our algorithm, however has fixed the co-articulation issue through the flow controls such as session manager, broadcaster, flowrouter, steady detector, etc. (components of OpenNI and NITE) by updating the sessions on any changes to the current depth data. 


\subsection{Usability}

The hands and line of sight (LoS) combination is considered by the authors in [68] as the interaction method. This method can lessen the fatigue that a one hand pointing interaction can cause and concurrently enhance the effectiveness of a task. Additionally, if we make use of the area cursor to the two hands based pointing and to the LoS based one hand pointing, greater results can be anticipated.

As for the multimodal interfaces, Cabral et al. [69] discuss numerous usability issues associated to the use of gestures as an input mode. A simplistically strong $2 \mathrm{D}$ computer vision based gesture recognition system was introduced by the authors and was successfully used for interaction in VR environments, such as CAVEs and Powerwalls. Three different scenarios were employed to test the interface: as a regular pointing device in a GUI interface, as a navigation tool, and as a visualization tool. Their results illustrated that it is more time consuming, as well as more fatiguing to complete simple pointing tasks than using a mouse. However, several advantages are revealed by the use of gestures as a substitute in multimodal interfaces. These include immediate access to computing resources using a natural and intuitive way, and that balances properly to joint applications, where gestures can be used infrequently.

A proposition by Villaroman et al. [70] suggests that using Kinect to classroom training on natural user interaction creates a prospect and innovative method. Examples are presented to demonstrate how Kinect-assisted instruction can be utilized to accomplish certain learning results in Human Computer Interaction (HCI) courses. Moreover, the authors have confirmed that OpenNI, in addition to its accompanying libraries, are adequate and beneficial in enabling Kinect-assisted learning activities. For students, 
Kinect and OpenNI offer a hands-on experience with its gesture-based, natural user interaction technology.

On the other hand, a promising interaction technique for distant displays is the free hand interaction as opposed to traditional input devices. The adaptation of three menu techniques for free hand technique is put forward by Bailly et al. [71]: Linear menu, Marking menu and Finger-Count menu. In their first study, which concentrates on Finger-Counting postures in front of interactive television and public displays, it demonstrates that the subjects do not opt for effective gestures. After improvement on their prototype, more precise and adequate gestures were used. This accomplishment was due to the fact that they developed a Finger-Count recognizer. As well, the experiment illustrated that Finger-Count is more mentally demanding than other techniques.

In a study on 3D applications using Kinect, Kang et al. [72] introduced a control method that naturally regulates the application with the use of distance information and joints' location information. Furthermore, the recognition rate was more successful, as well as the use of the proposed gestures in the $3 \mathrm{D}$ application, which was $27 \%$ quicker than a mouse.

Code Space, introduced by Bragdon et al. [73], is a system that combines touch + air gesture hybrid interactions to jointly carry small developer group meetings. This method enables access, control and sharing of information through several different devices such as multi-touch screen, mobile touch devices, and Microsoft Kinect sensors. In a formative study, professional developers were positive about the interaction design, and most felt that pointing with hands or devices and forming hand postures are socially acceptable. 
A gesture user interface application, titled Open Gesture, is available for standard tasks, for instance making telephone calls, operating the television, and executing mathematical calculations [74]. This prototype uses a television interface to carry out various tasks by using simple hand gestures. Based on a usability evaluation, Bhuiyan and Picking [74], recommend that this technology can improve the lives of the elderly and the disabled users by creating more independence while some challenges still remain to be overcome. During a study, on touch-free navigation through radiological images, analyzed by Ebert et al. [75], ten medical professionals tested the system by rebuilding a dozen images from a CT data. The experiment measured the response period and the practicality of the system compared to the mouse/keyboard control. An average of ten minutes was required for the participants to be at ease with the system. The response time was $120 \mathrm{~ms}$, and the image recreation time using gestures was 1.4 time longer than using mouse/keyboard. However it does remove the potential for infection, for both patients and staff. Moreover, users with OsiriX experience, who rated the system 3.4 out of 5 in comparison to the mouse/keyboard, completed the tasks considerably easier while using a mouse/keyboard.

Designing a suitable user interface for the following usability studies is crucial. Novice users can learn WIMP user interfaces easily, as they are very good at abstracting workplaces due to their analogous paradigm to documents like paper sheets or folders. Having a rectangular region on a $2 \mathrm{D}$ flat screen makes them preferable to system developers while their generality also makes them a good fit in multitasking environments. 
In order to have more accurate results in our usability study, we have designed a simple and minimalistic as possible simulated desktop interface with neutral colors to reduce user error or bias, while we focused on common desktop tasks to be relatively general.

Our algorithm also recognizes point finger and thumb, with a possibility of orderly detecting all five fingers.

Moreover, natural gestures definition and recognition methods that we have created in our research investigate new patterns for communication.

Furthermore, we have used more features in our usability study than those have been studied in above mentioned related works. Through a comprehensive and hypothetical user experiment we have statistically analyzed and compared our natural defined gestures (finger and arm) to each other and also to the conventional input devices (mouse/keyboard), in two different situations (small and big-screen displays), and in two different settings (simple and complex tasks) for precision, efficiency, ease-of-use, funto-use, fatigue, naturalness, and overall satisfaction. We believe that this study develops the models and theories of interaction. 


\section{Chapter 3: Gesture Recognition}

\subsection{Selecting Gestures}

Our research has two main phases:

1- Arm gestures:

a) Designing a UI to be controlled by mouse and some arm gestures.

b) Developing arm gesture recognition module.

c) Running a user experiment to compare/analyze the new applied approaches and the possible future improvements in remotely controlling of a customized system by arm gestures and its conventional competitor of mouse/keyboard input devices.

2- Finger gestures:

a) Designing a UI to be controlled by some arm gestures (already designed in phase 1) and some finger gestures.

b) Developing finger gesture recognition module. 
c) Running a user experiment to compare/analyze the new applied approaches and the possible future improvements in remotely controlling of a customized system by finger gestures and arm gestures as the input devices.

\subsubsection{Finger set vs. Arm/Hand set}

\subsubsection{Predefinition}

In our first gesture design study we defined the finger and arm gestures as shown in Table 3.1.a) and b). 
Table 3.1.a) Initial design for finger set vs. arm/hand set.

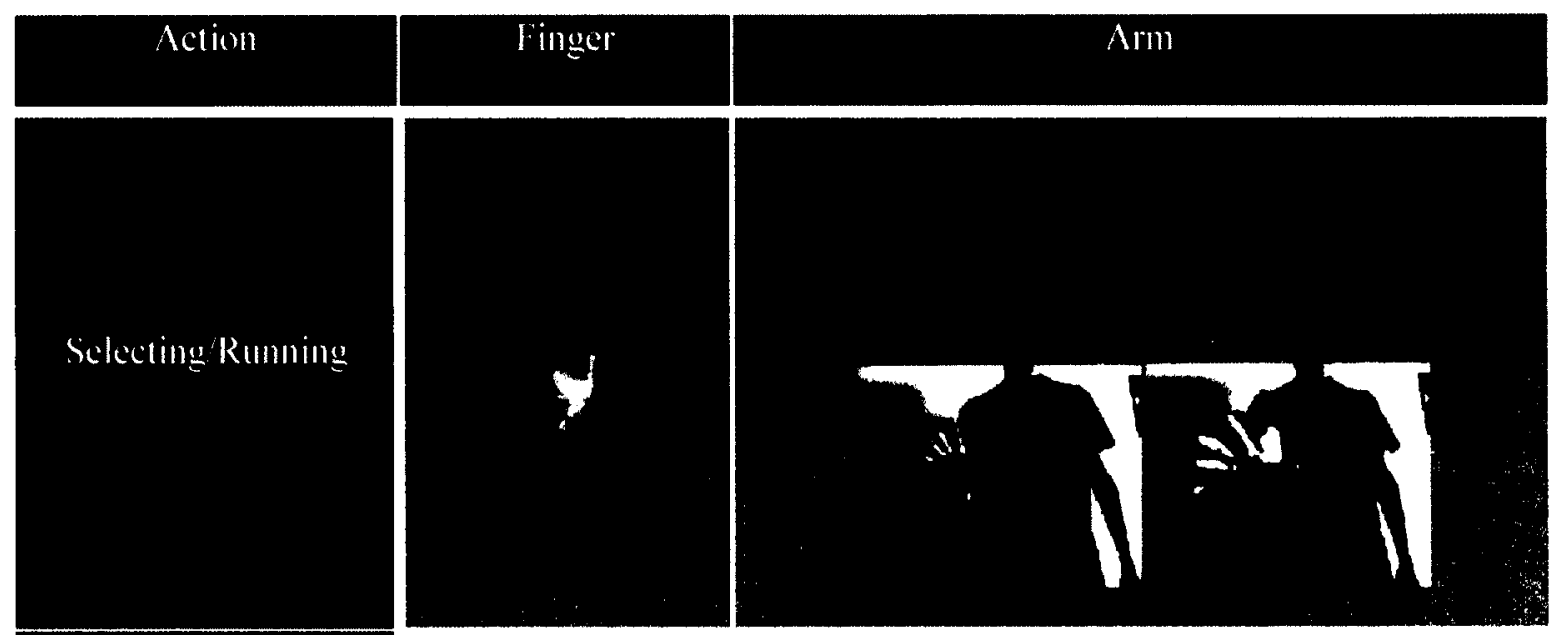

Possibilities:

Moving finger/hand in space (pointer follows the hand)

Mor ing cursor
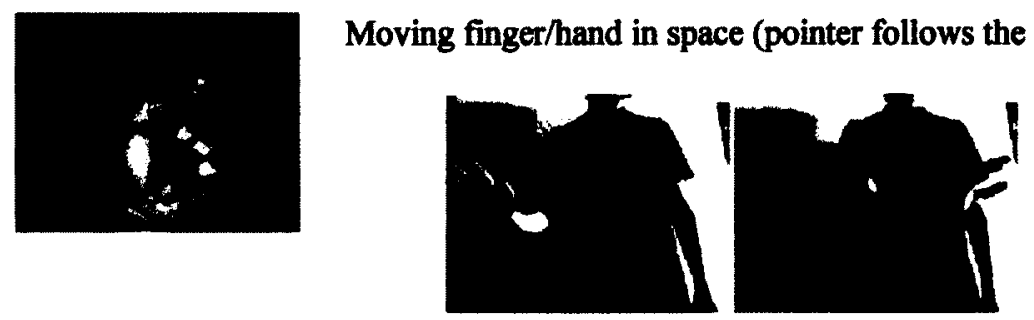

(irabbing/Dragging

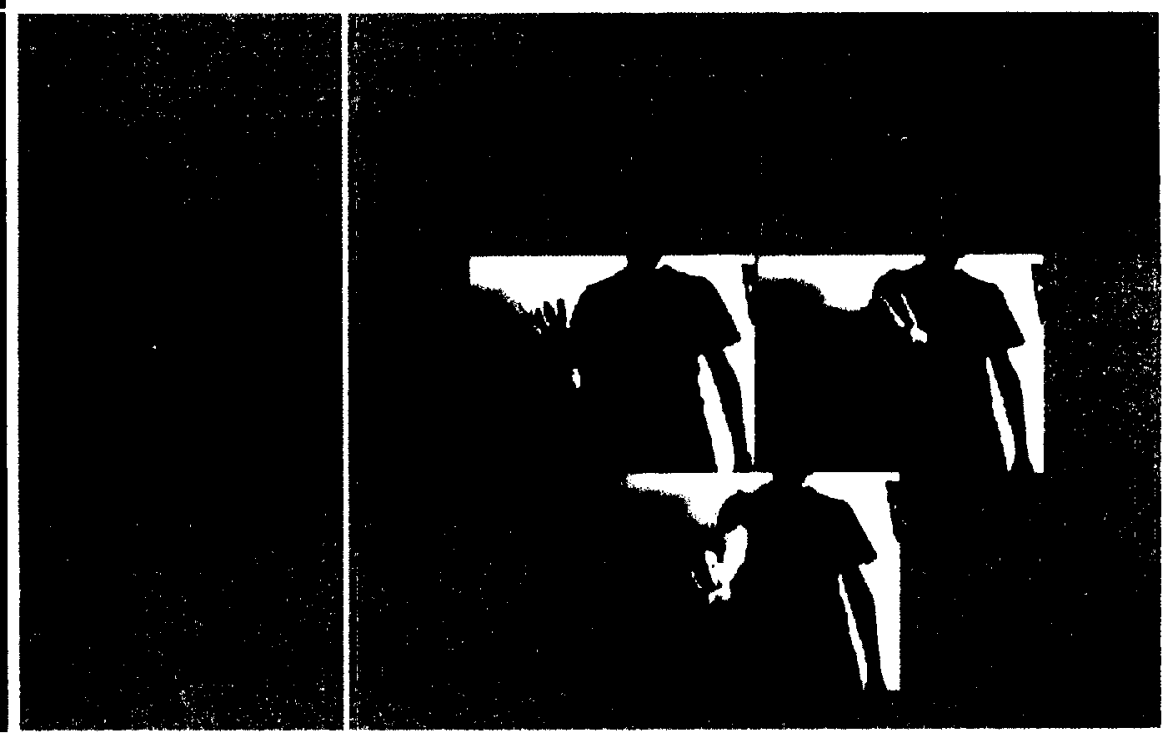


Table 3.1.b) Initial design for finger set vs. arm/hand set (continued from last page).

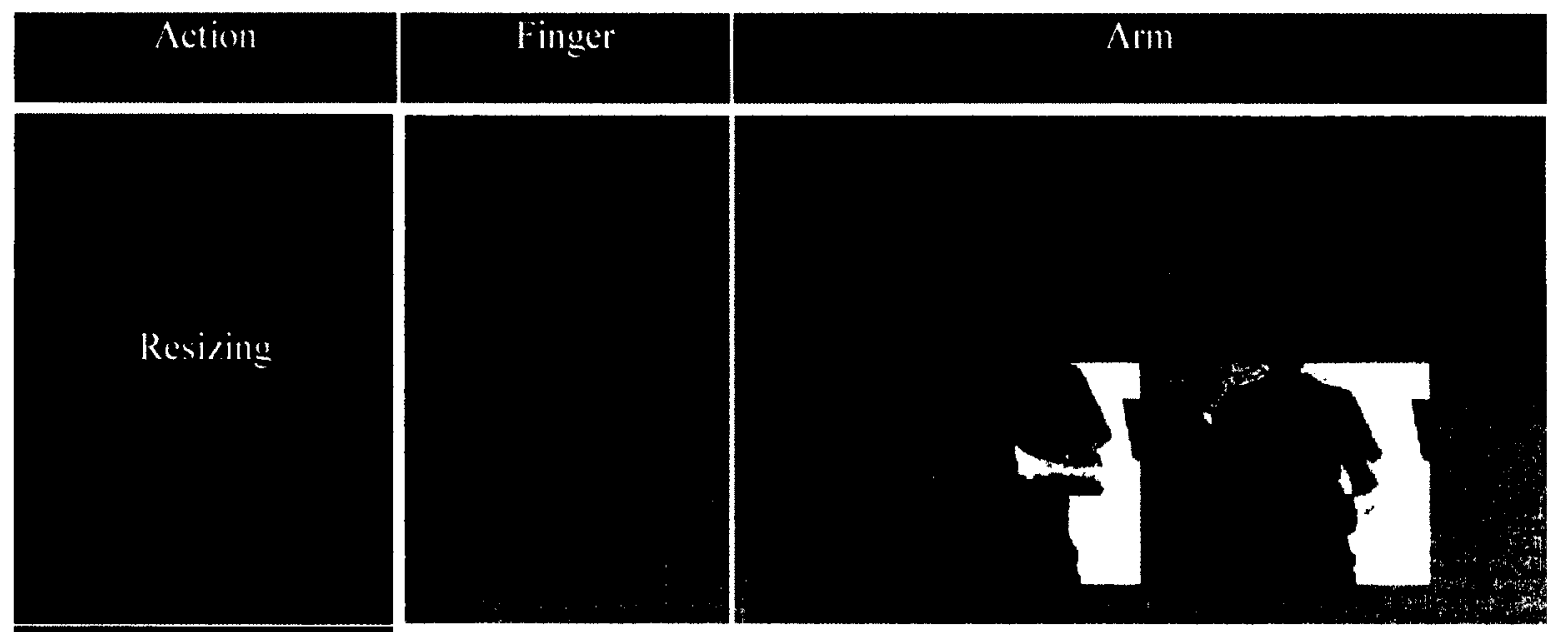

Possibilities:

A finger/hand gesture combined with whole arm

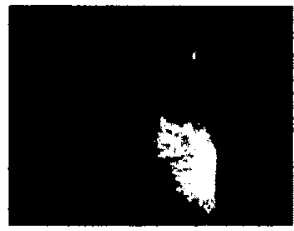
movement

Scrolling

Deselecting/Closing

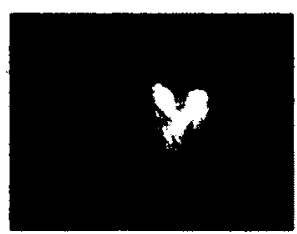

Possibilities:

Perhaps a fist or the same idea as having a second arm motion, or pulling the hand backward to deselect/close 


\subsubsection{Final Definition}

Studying the natural body languages and considering some predefined features of applied APIs, e.g. OpenNI and NITE, led us to finalize our gesture definitions as shown in Table 3.2 .

Table 3.2. Final design option for finger set vs. arm/hand set

(uv: user view, cv: camera view).

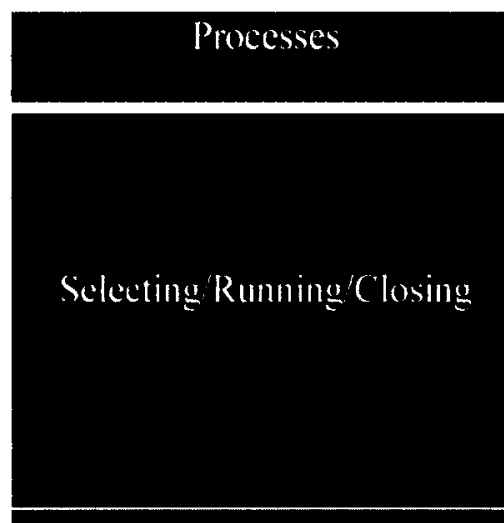

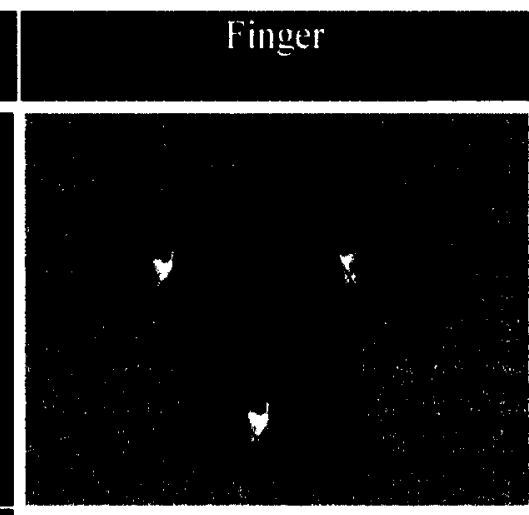

Finger moving

Moring colser

Crabbing Resiring

Control releasing
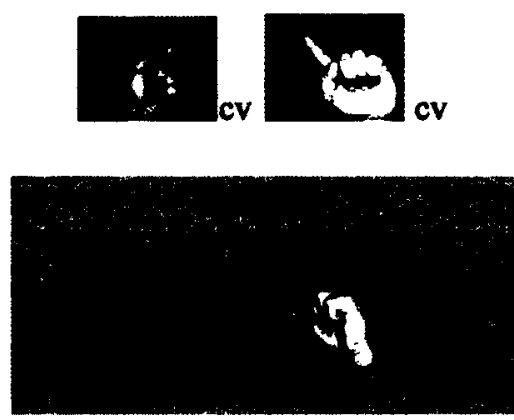

Multi fingers

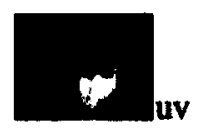

Listra options

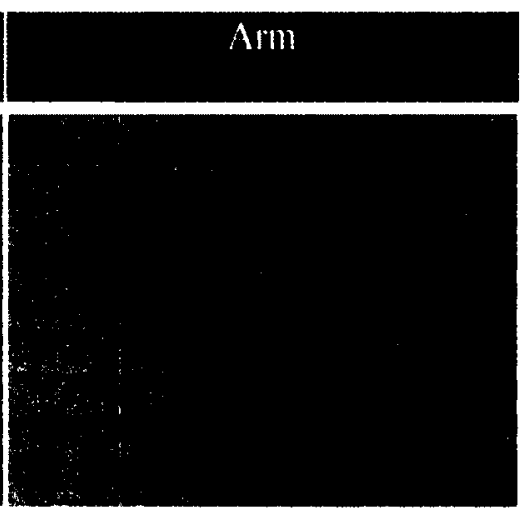

Hand moving

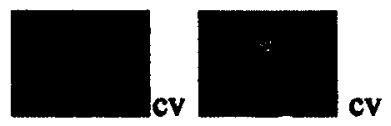

Open palm
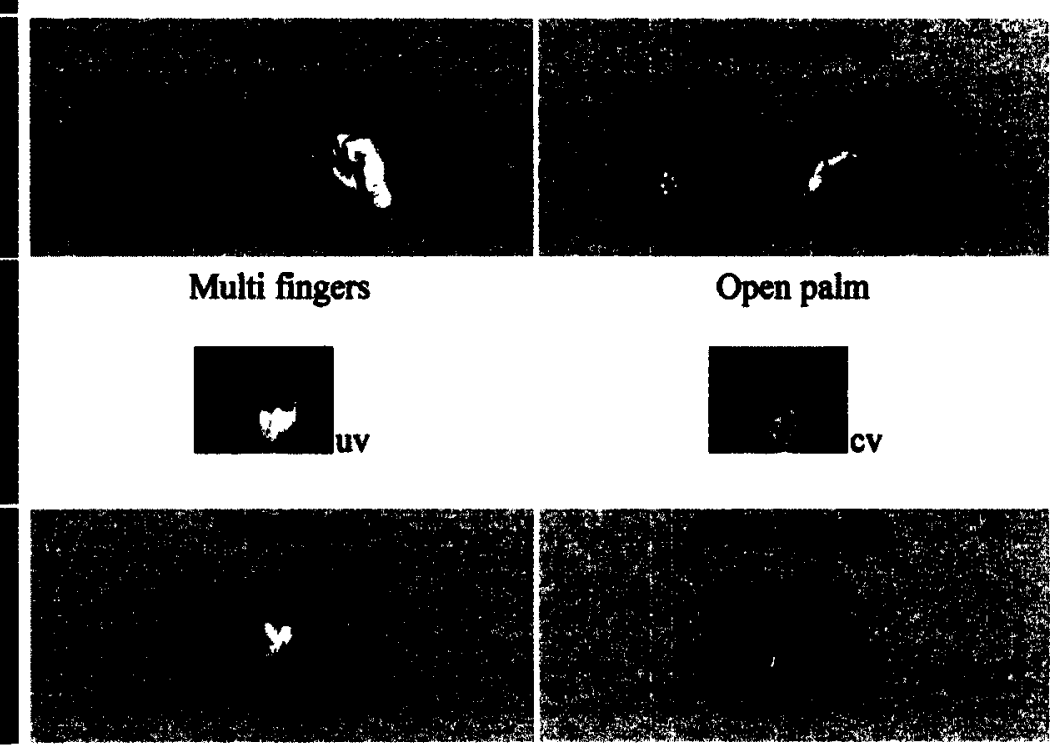


\subsection{Fingertips Detection}

We use OpenCV, specially in coding our prototype for phase 2 (finger gestures) where the fingertips are needed to be recognized. This will be done by the following algorithm:

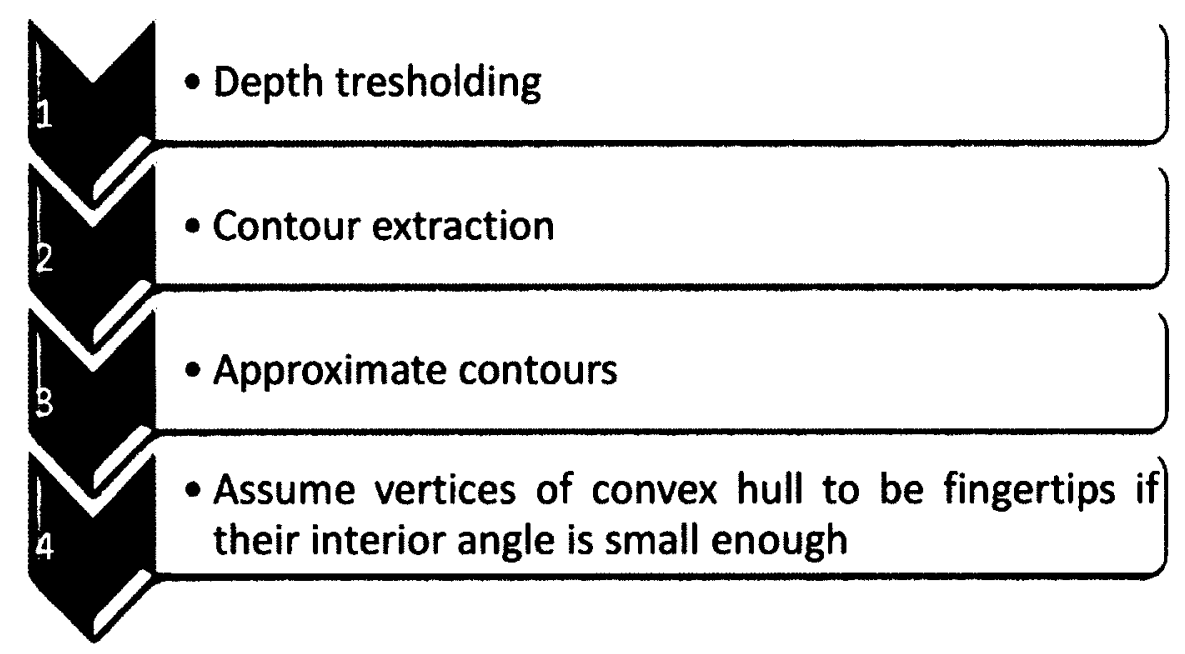

Figure 3.1. Fingertips detection algorithm in OpenCV.

The method of thresholding the depth map in OpenCV is as follows:

1- Store depth map in an array.

2- Iterate over each pixel in the array.

3- Create a binary image: set all pixels outside of the depth range to 0 and all of those within the depth range to 1 .

4- Find contours/connected components.

5- Detect convexity hull/defects. 
The convexity defects method is not completely robust in that the defects in the convex hull will change from frame to frame depending on hand orientation/position. In the case of fingertip tracking, we would need to have our hand in a relatively stable position over time for the convexity defects to remain stable.

Cropping the depth map can help in order to remove the wrist from the depth map which can cause unwanted defects. In terms of hand gesture recognition, cropping the wrist out of the frame can also help in improving accuracy.

Following the algorithm in Figure 3.6, we have calculated the threshold angle through the following equation:

$$
\text { angle }=\cos ^{-1} \frac{\left(v_{x} \times v_{x}^{\prime}\right)+\left(v_{y} \times v_{y}^{\prime}\right)}{\|v\| \times|| v^{\prime} \|}
$$

With a robust method and using convexity defect, if needed we can also approximately detect the fingers' joints using the location of palm's centre point, the fingertips coordinates, and the distance/angle between fingertips in different positions, along with bringing the respective depth of these points to the computation.

Our algorithm also recognizes point finger and thumb, with a possibility of orderly detecting all five fingers.

\subsection{Algorithm}

We have designed an algorithm as shown in Figure 3.4, to control our user interface objects by recognizing the arm and finger gestures (Circle and Push are replaced by Pinch and Tap in finger algorithm) during the controlling sessions of OpenNI and NITE APIs. 


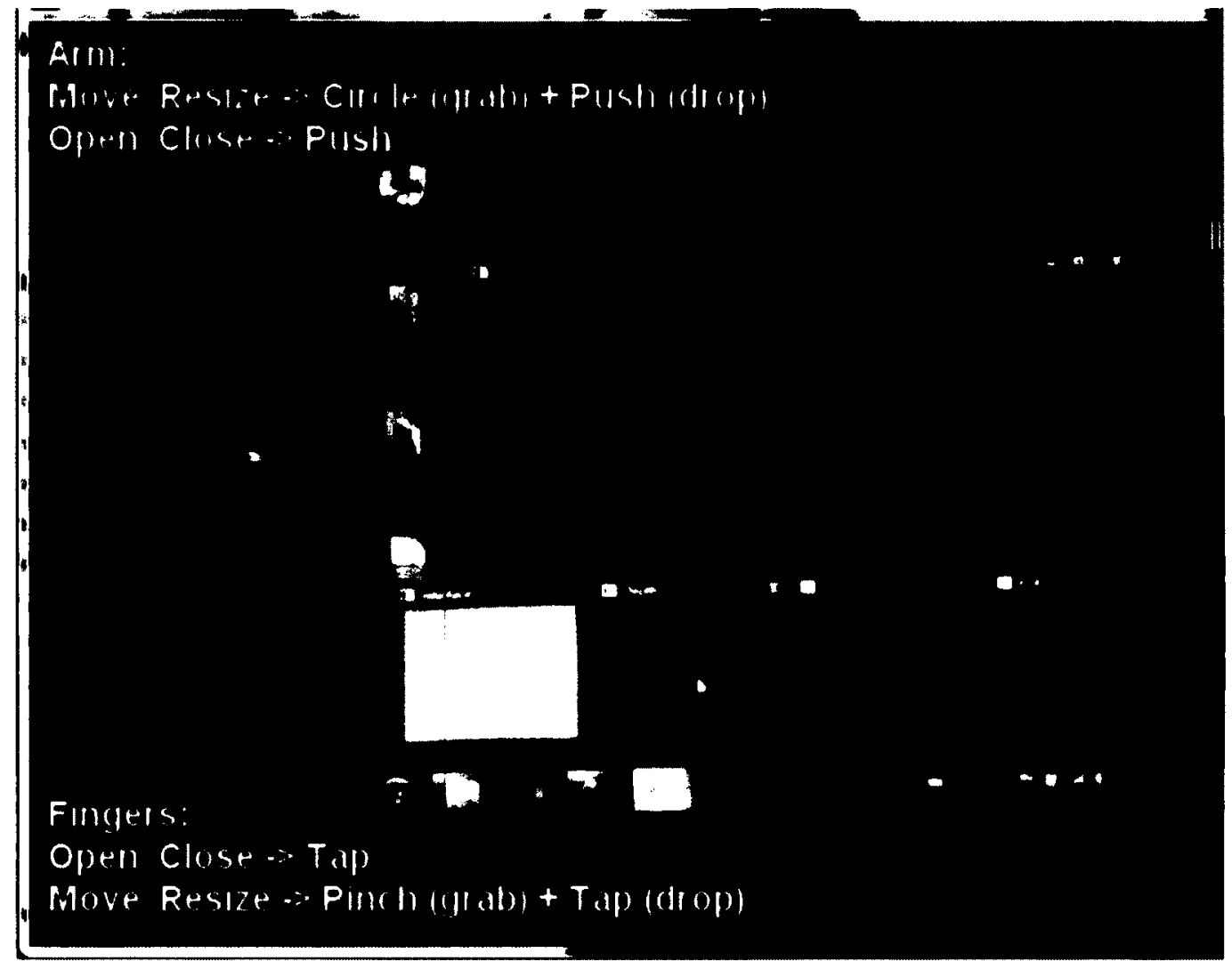

Aim

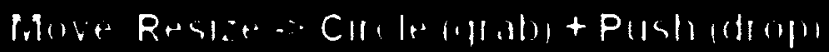

Open ClM... PISH
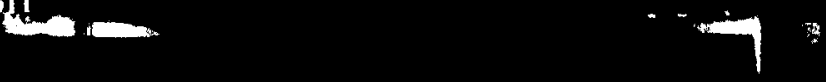

li

$\mathbf{3}$

Fingeres

Open Close $\therefore$ Tap

Move Resize $\therefore$ PInch (glab) + Tap) (dIOp)

Figure 3.2. Finger/Arm detection steps. 

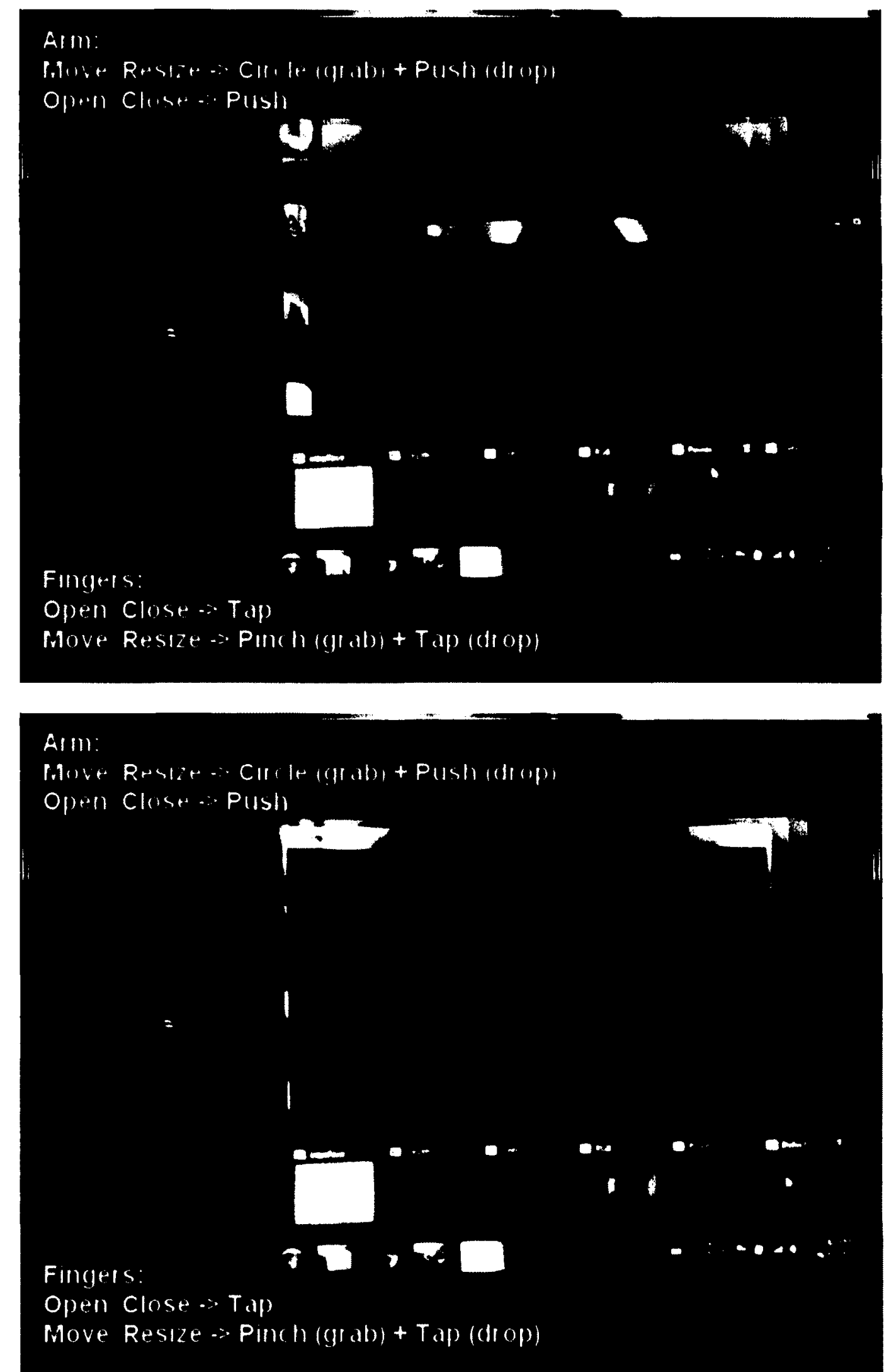

Figure 3.3. Finger detection steps. 


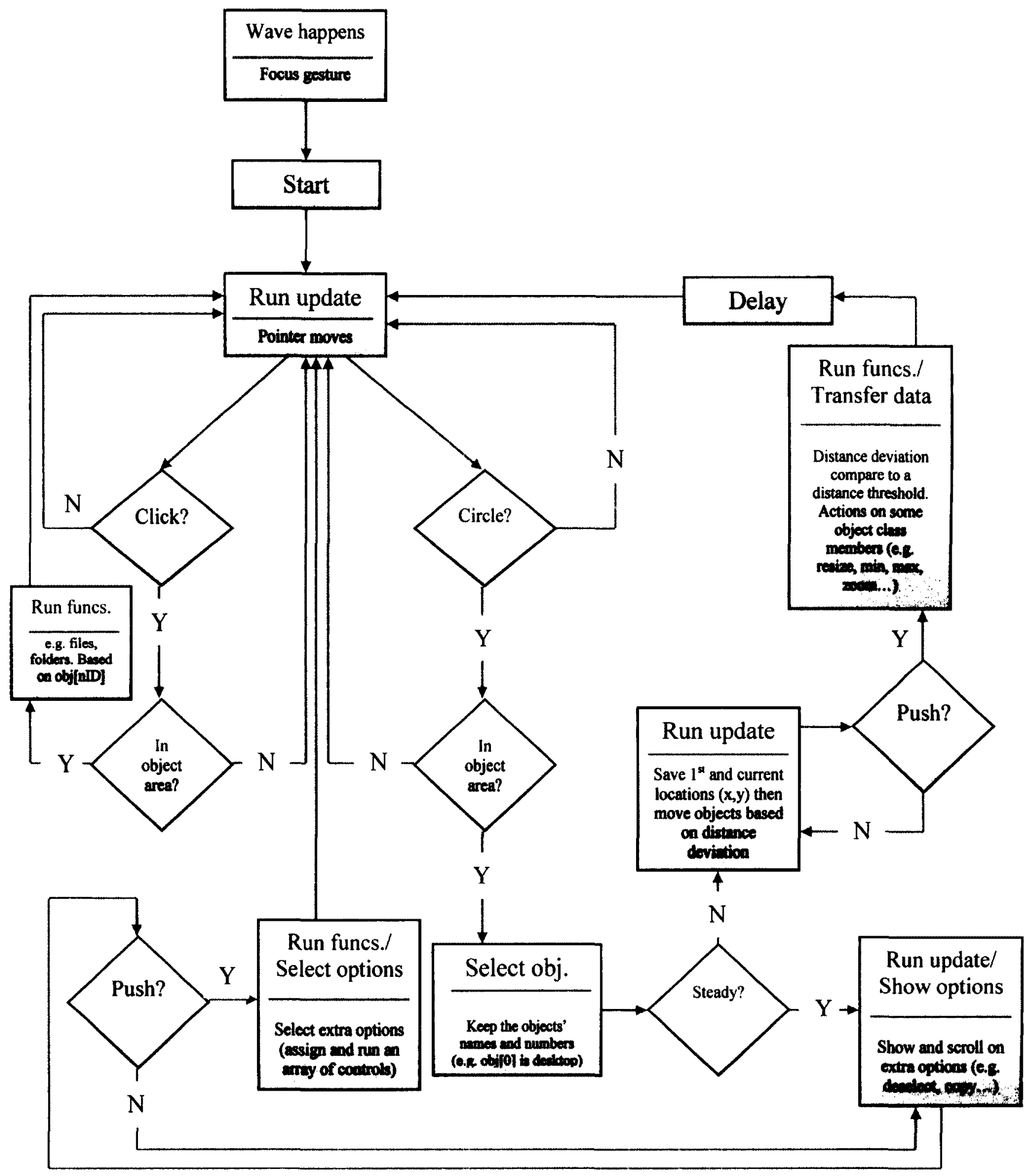

Figure 3.4. The algorithm controlling UI using arm gestures recognition (similar to finger gestures with replacing Push and Circle to Tap and Pinch). 
Each action can control some object's class members (e.g. centre of object, file, folder, resize, minimize, maximize, zoom, close, scroll-bar, and some extra options such as copy, rename, etc.).

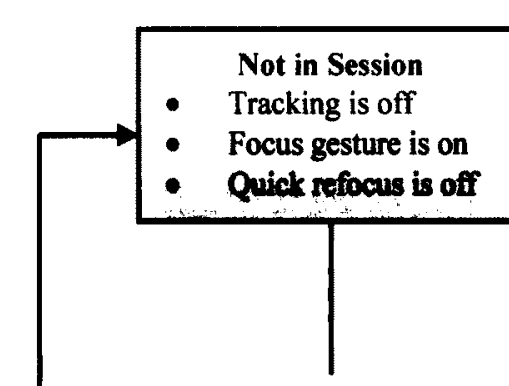

Focus gesture recognized

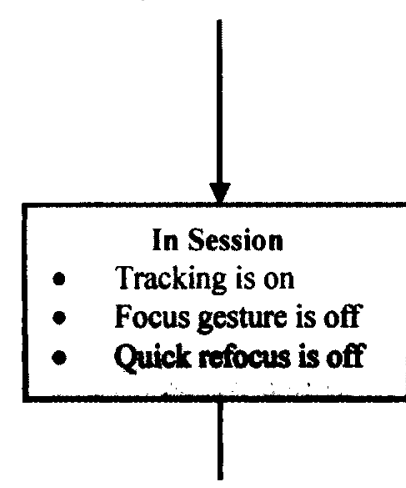

No active points

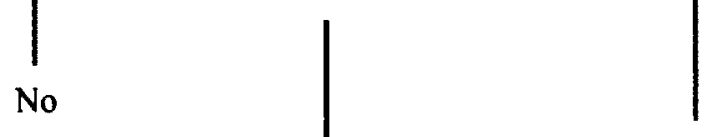

Focus gesture recognized

Quick refocus recognized

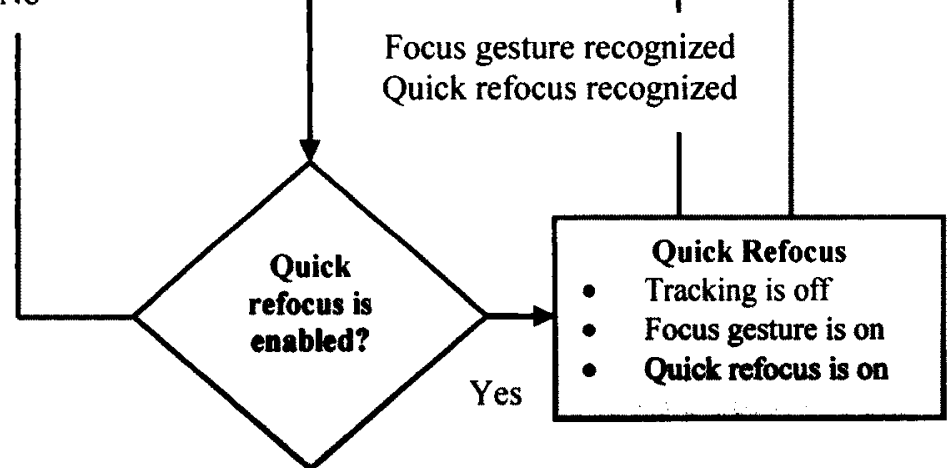

(a)

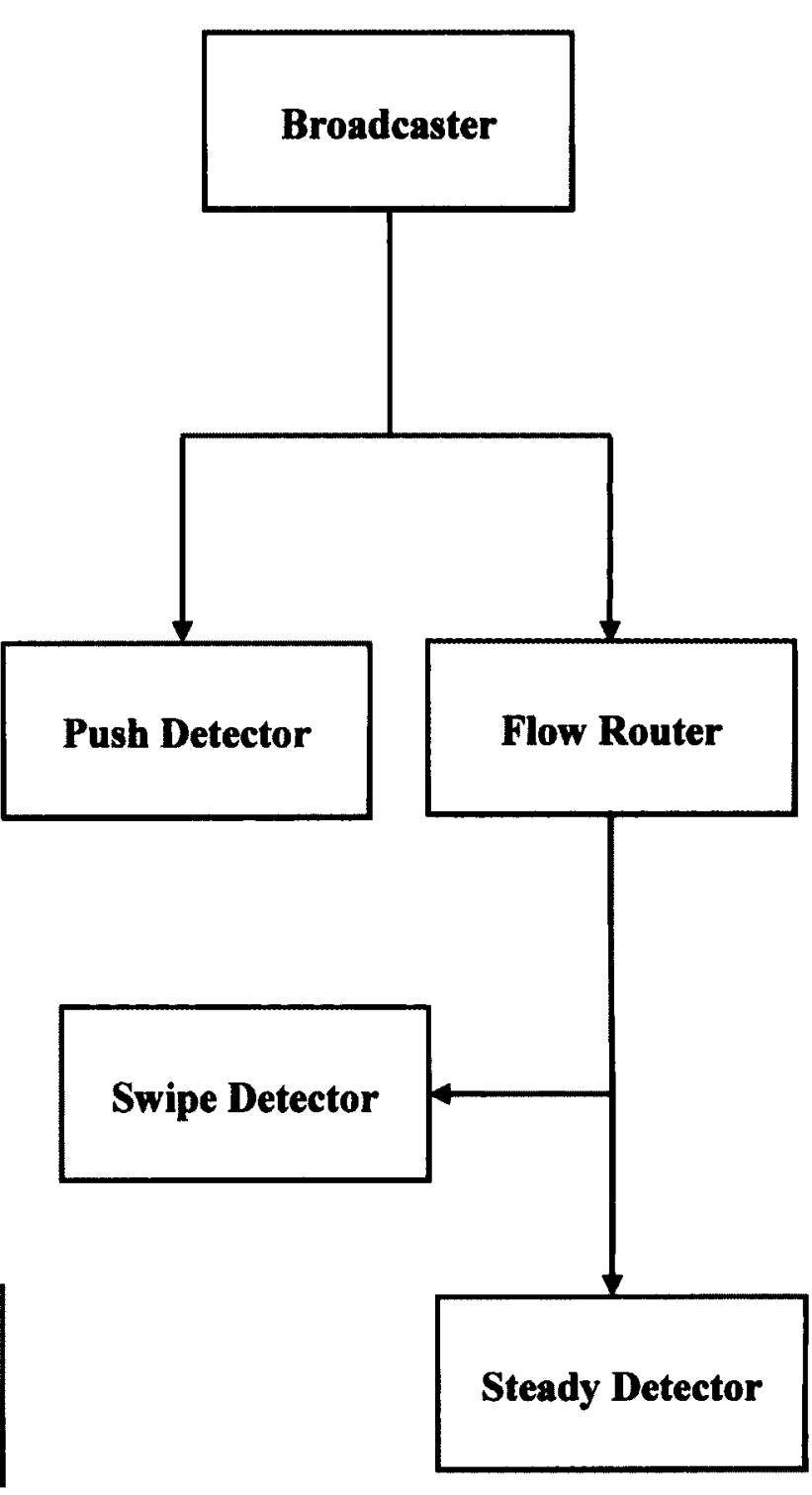

(b)

Figure 3.5. NITE algorithm to detect arm gestures: (a) session state automation,

(b) compound control (re-created based on [62]). 


\section{Chapter 4: UI and Experiment Design}

\subsection{Settings}

We have run the system under the following settings/ availabilities:

1- Operating System: 32-bit or 64-bit Windows XP, Vista, 7 SP 1. However the system itself has the capability of working under multi platforms.

2- Camera: Microsoft Kinect depth camera, or PrimeSense depth camera, located at about one meter (finger phase) and one to three meters from the user (arm phase) for the best results.

3- Development tools: Microsoft Visual Studio $(\mathrm{C}++)$, OpenNI, NITE, OpenCV, and Allegro libraries.

4- Display: To compare a traditional input device with our $\mathrm{HCl}$ natural input device, availability of a video projection system or a big screen HDMI TV is needed. In our case we have used a projector (resolution: 480p, screen size: 92 inches) located at about five to eight meters from the user. 


\subsection{Gestures and Actions}

In this prototype we have provided some actions such as run, move, resize, or close which can be performed by recognizing our defined gestures e.g. push, or a combination of gestures e.g. pinch + move + tap, as shown in Table 4.1 .

Table 4.1. (a) Arm and (b) Finger gestures' definitions, mouse analogies, and actions.

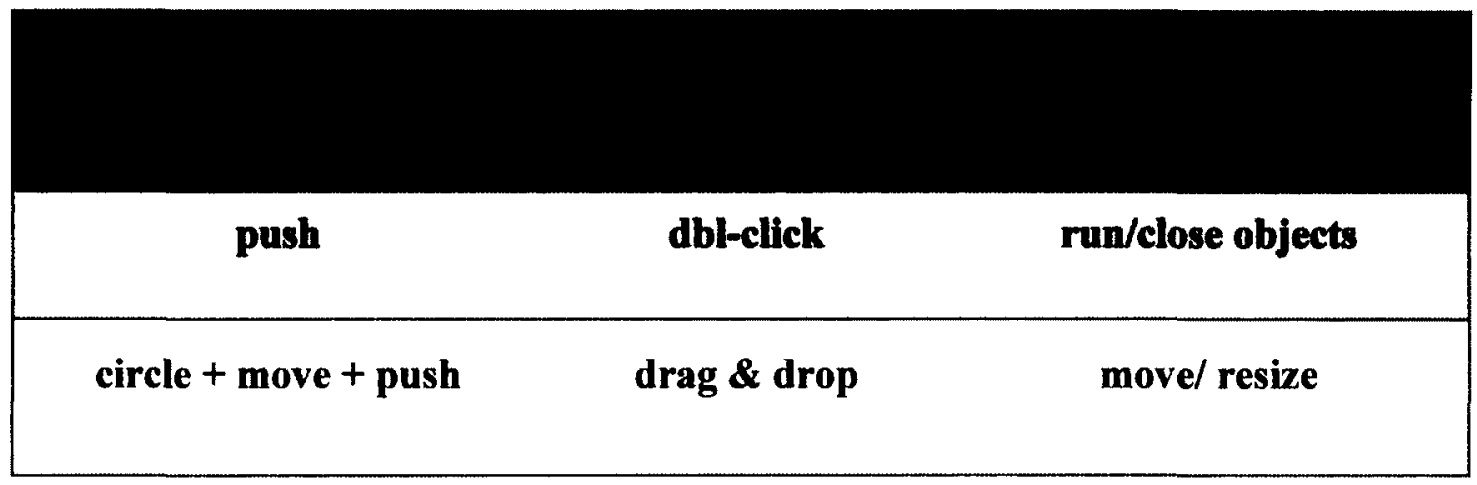

(a)

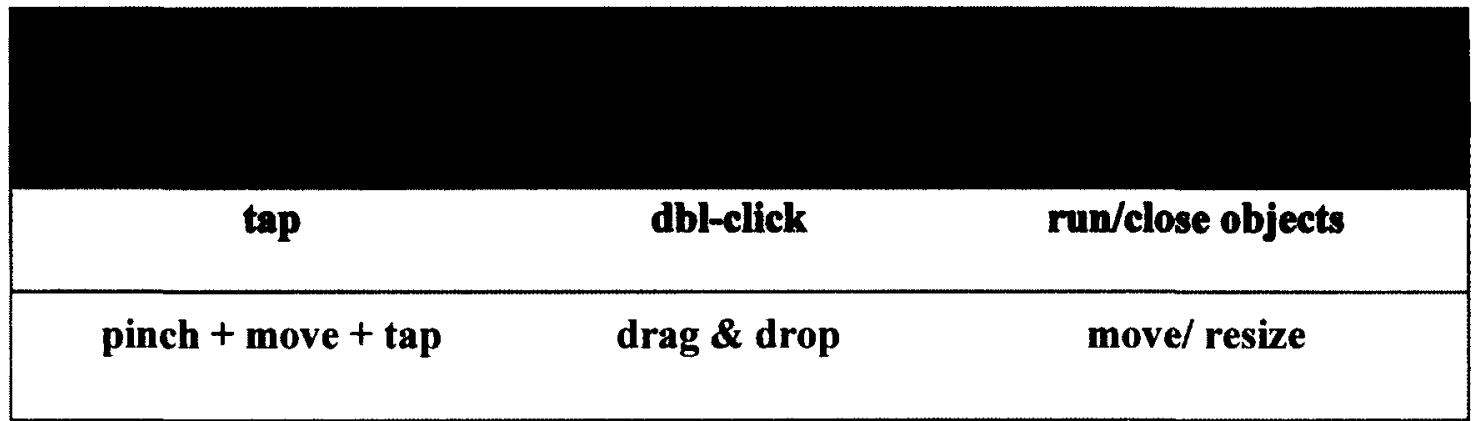

(b)

After detecting the hand and the palm centre in OpenNI (through a process of creating the context, depth/image/hand generator nodes, session manager, initializing/registering/updating the sessions/controls, and 3D mapping), we have used the predefined classes of gestures in NITE, for "circling" and "pushing". 
After detecting the fingertips in OpenCV, as explained earlier, and through a process of 3D mapping we can extract the fingertip's coordinates. The "tapping" gesture in finger phase has been defined based on the depth change of point finger's tip $\left(\boldsymbol{z}_{\boldsymbol{p}}\right)$ comparing to the centre of hand's/palm's depth $\left(\boldsymbol{Z}_{\boldsymbol{h}}\right)$, and a proper threshold $(\boldsymbol{D})$.

$$
\text { Tapping happens when : } z_{h}-z_{p}>D
$$

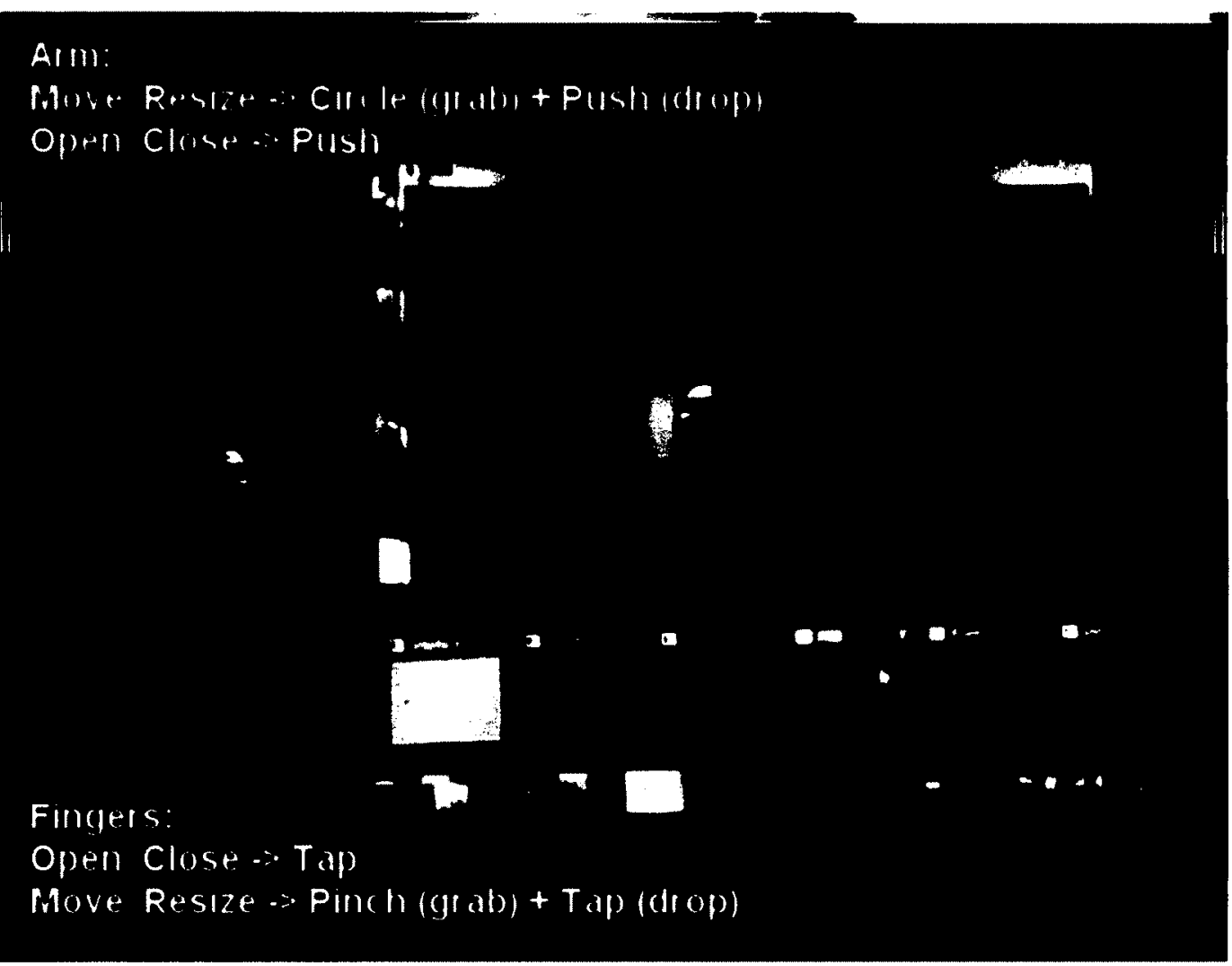

Figure 4.1. Finger tapping gesture. 
The "pinching" gesture in finger phase has been defined based on the distance between the point finger's tip $\left(x_{p}, y_{p}, z_{p}\right)$ and thumb's tip $\left(x_{t}, y_{t}, z_{t}\right)$.

$$
\text { Pinching happens when : }\left\{\begin{array}{l}
x_{p}-x_{t}<\varepsilon \\
y_{p}-y_{t}<\varepsilon \\
z_{p}-z_{t}<\varepsilon
\end{array}\right.
$$

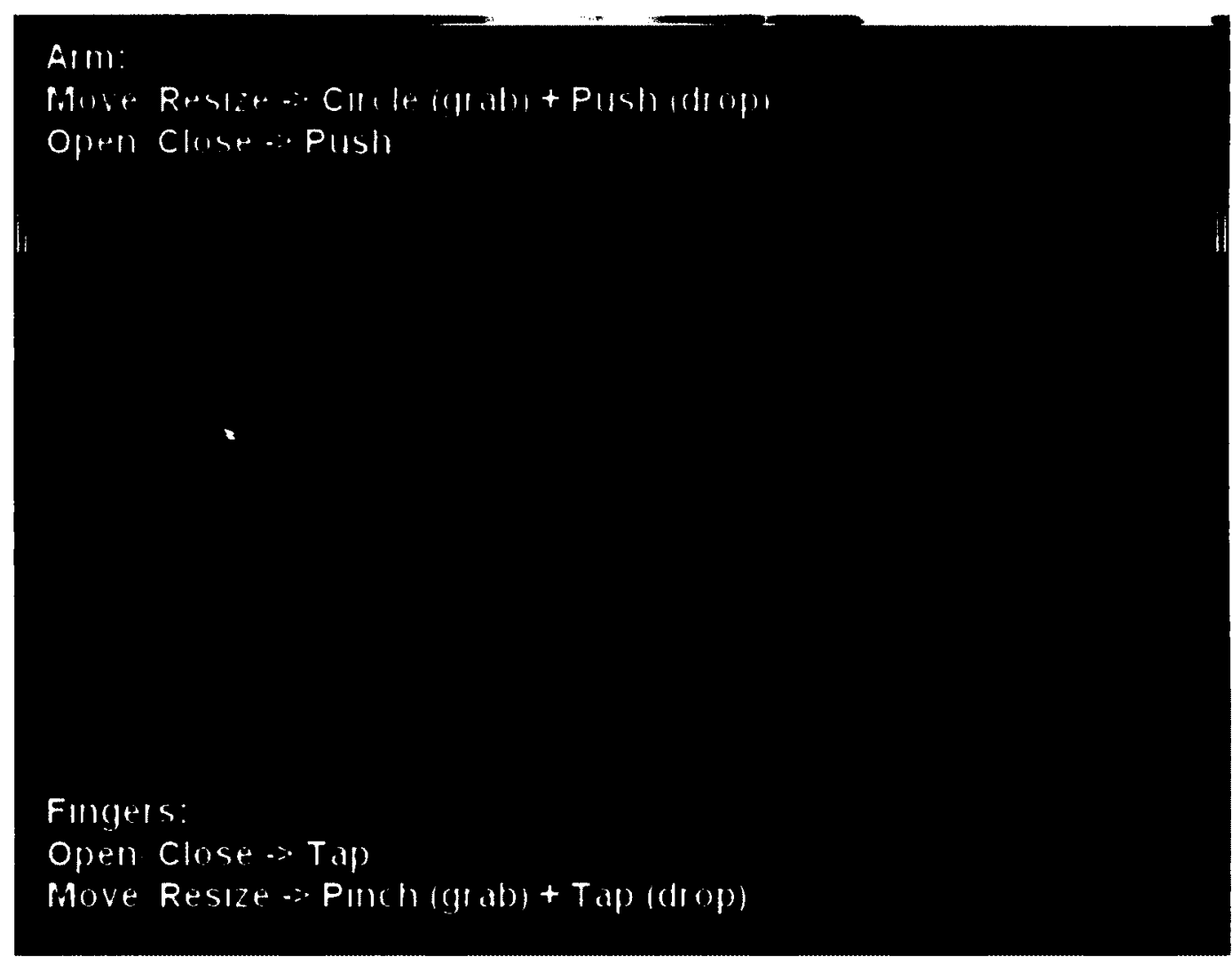

Figure 4.2. Finger pinching gesture. 


\subsection{User Experiment}

Variables, evaluation criteria, questions, and measurements are listed in the following tables:

Table 4.2. List of variables in our user experiment.

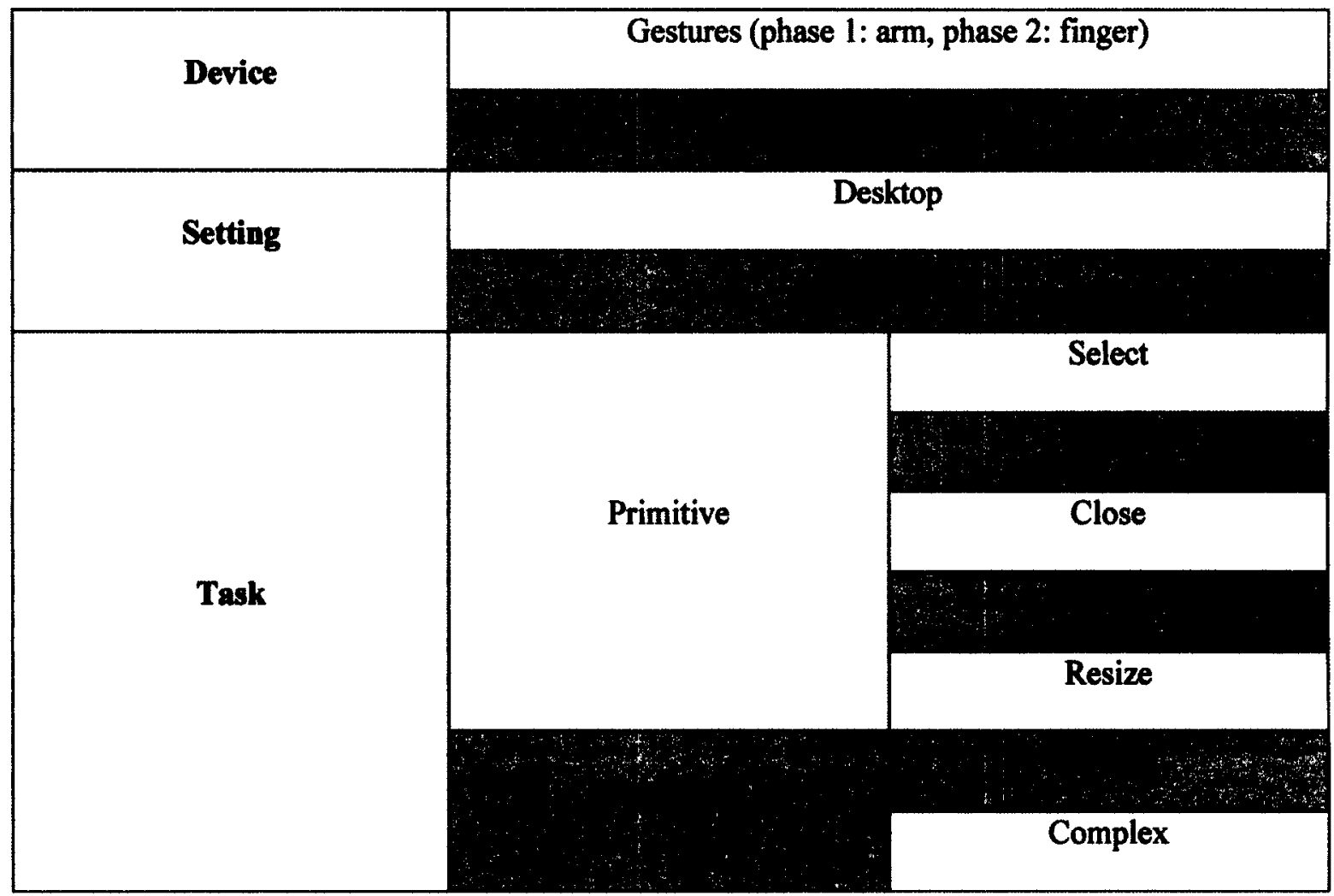


Table 4.3. Evaluation criteria and their replying contexts (questions and measurements).

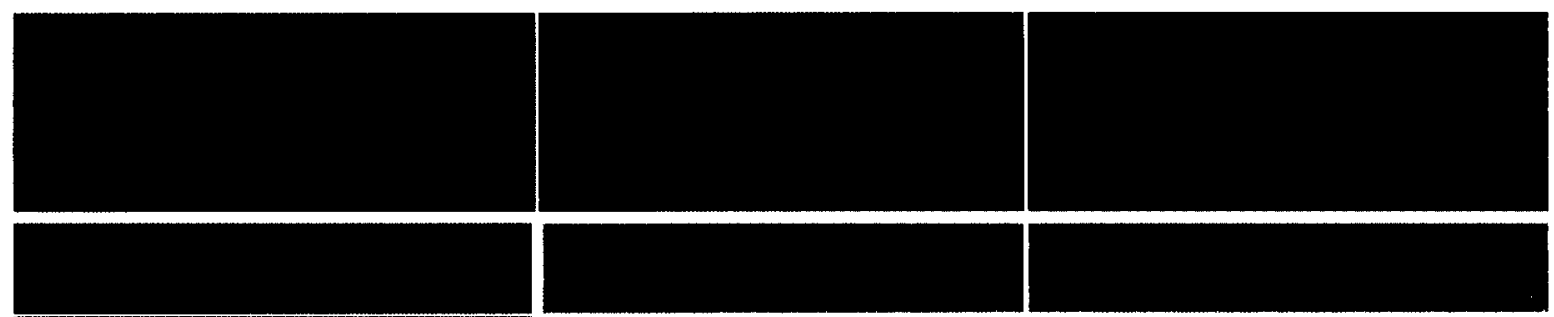

How much fatigue did it

cause?

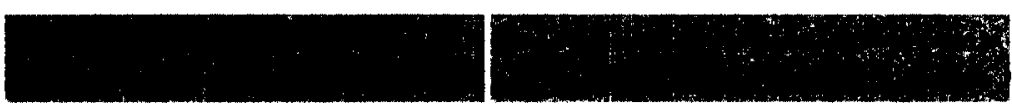

How pleasant was it?

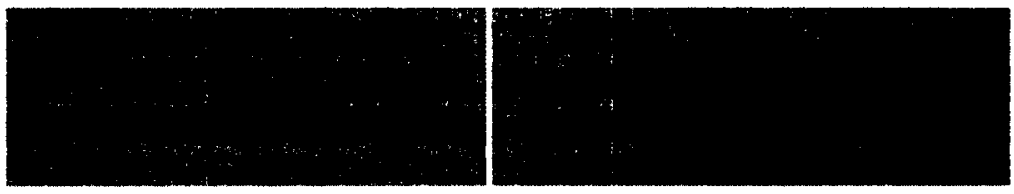

Required Time

\subsubsection{Process}

\subsubsection{Training session}

Phase 1 - Training session using mouse and arm:

- Thirty minutes of practicing the tasks. This is for two reasons:

1- To get used to the application in order to run the main test precisely.

2- We use this part to get the users' evaluation for some questions, e.g. arm/mouse fatigue, naturalness, etc. 
- Try the following four primitive tasks to get used to the application in order to run the test session precisely:

a. Open window (Run icon)

i. Mouse: Left-click on the icon and press enter.

ii. Arm: Push towards the icon.

b. Close window

i. Mouse: Point on "X" sign on the window, and left-click on it.

ii. Arm: Point on "X" sign of the window, and push towards it.

c. Move objects (icon/window)

i. Mouse:

1) On icon: Left-click on the icon, and move a bit simultaneously (drag), then release the mouse key to drop it.

2) On window: Left-click on the title bar (name tag) of the window, and move a bit simultaneously (drag), then release the mouse key to drop it.

ii. Arm:

1) On icon: Draw a circle around the icon, and move a bit (drag), then push to drop it.

2) On window: Draw a circle around the middle of the title bar (name tag), and move a bit (drag), then push to drop it.

d. Resize window

i. Mouse: Left-click on the bottom-right corner of the window, and move a bit simultaneously (drag), then release the mouse key. 
ii. Arm: Draw a circle around bottom-right corner of the window, and move a bit (drag), then push to release.

\section{Phase 2 - Training session using finger and arm:}

- Thirty minutes of practicing the tasks.

- Try the following four primitive tasks to get used to the application in order to run the test session precisely:

a. Open window (Run icon)

i. Finger: Tap towards the icon.

ii. Arm: Push towards the icon.

b. Close window

i. Finger: Point on "X" sign on the window, and tap towards it.

ii. Arm: Point on "X" sign of the window, and push towards it.

c. Move objects (icon/window)

i. Finger:

1) On icon: Pinch towards the icon, and move a bit (drag), then tap to drop it.

2) On window: Pinch towards the middle of the title bar (name tag), and move a bit (drag), then tap to drop it.

ii. Arm:

1) On icon: Draw a circle around the icon, and move a bit (drag), then push to drop it. 
2) On window: Draw a circle around the middle of the title bar (name tag), and move a bit (drag), then push to drop it.

d. Resize window

i. Finger: Pinch towards the bottom-right corner of the window, and move a bit (drag), then tap to release.

ii. Arm: Draw a circle around bottom-right corner of the window, and move a bit (drag), then push to release.

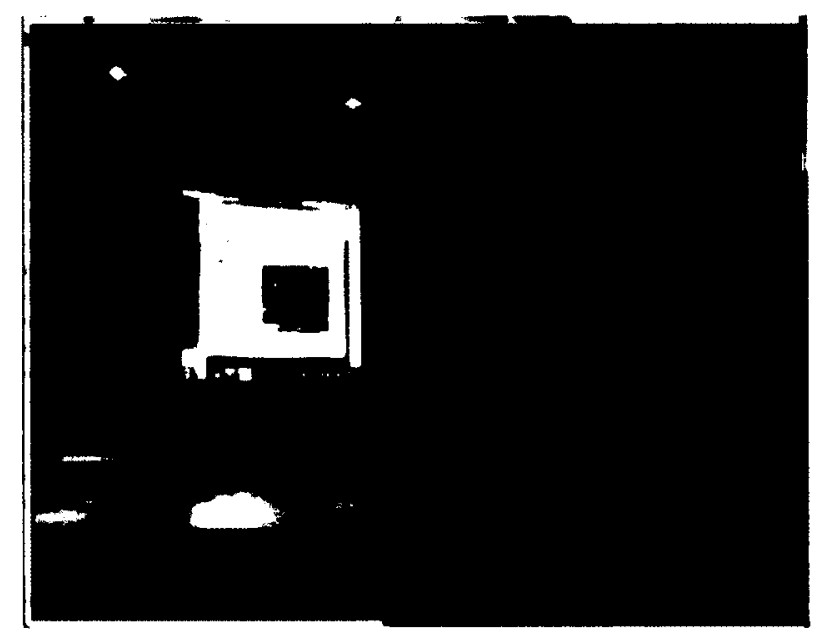

Figure 4.3. Training session.

\subsubsection{Test session}

Comparing two tasks (simple and complex), two devices (mouse and arm, or arm and finger), and two types of screens (desktop and big-screen) makes eight states of sessions in each phase. Some test session samples are as following: 
Test Session 1: Simple task, using mouse, on desktop

\section{Task}

The users are asked to include their physical actions along with the verbal definitions of each action (underlined words), e.g. left-click or enter, in order for the testing person to count the number of errors in gesture recognition.

1. Start the program.

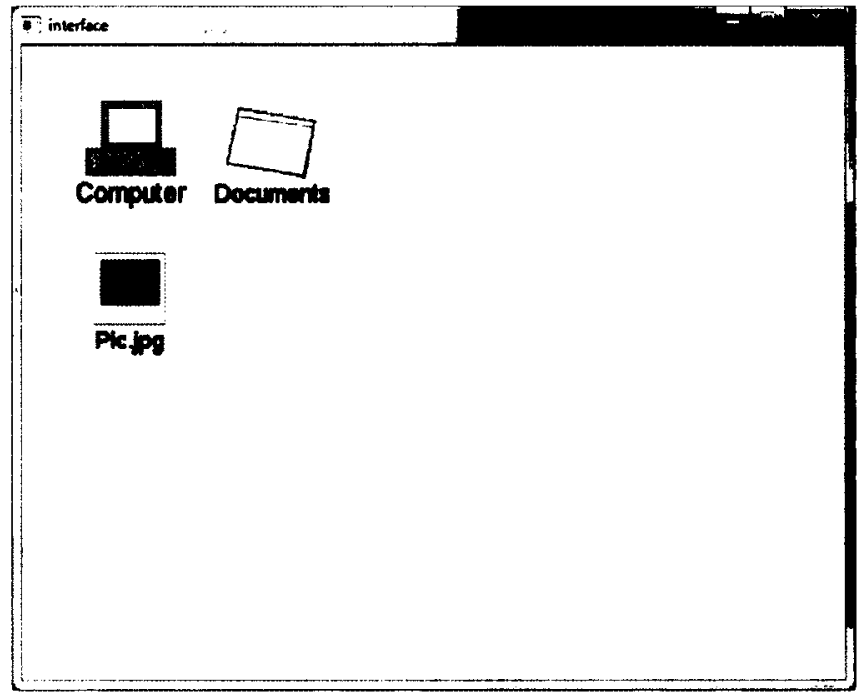

Figure 4.4. UI: start session.

2. Left-click on "Pic.jpg" icon and press enter. The window "Pic.jpg" is opened.

3. Left-click on bottom-right corner of the window, and move a bit simultaneously (drag), then release the mouse key. The window "Pic.jpg" is resized.

4. Left-click on the title bar (name tag) of the window, and move a bit simultaneously (drag), then release the mouse key to drop it. The window "Pic.jpg" is moved.

5. Point on " $X$ " sign on the top-right corner of the "Pic.jpg" window, and left-click on it. The window "Pic.jpg" is closed. 
Test Session 3: Simple task, using arm, on desktop

\section{Task}

The users are asked to include their physical actions along with the verbal definitions of each action (underlined words), e.g. push or circle in order for the testing person to count the number of errors in gesture recognition.

1. Wave (about five times fast waving) to start the program. The pointer appears.

2. Push towards "Pic.jpg" icon. The window "Pic.jpg" is open.

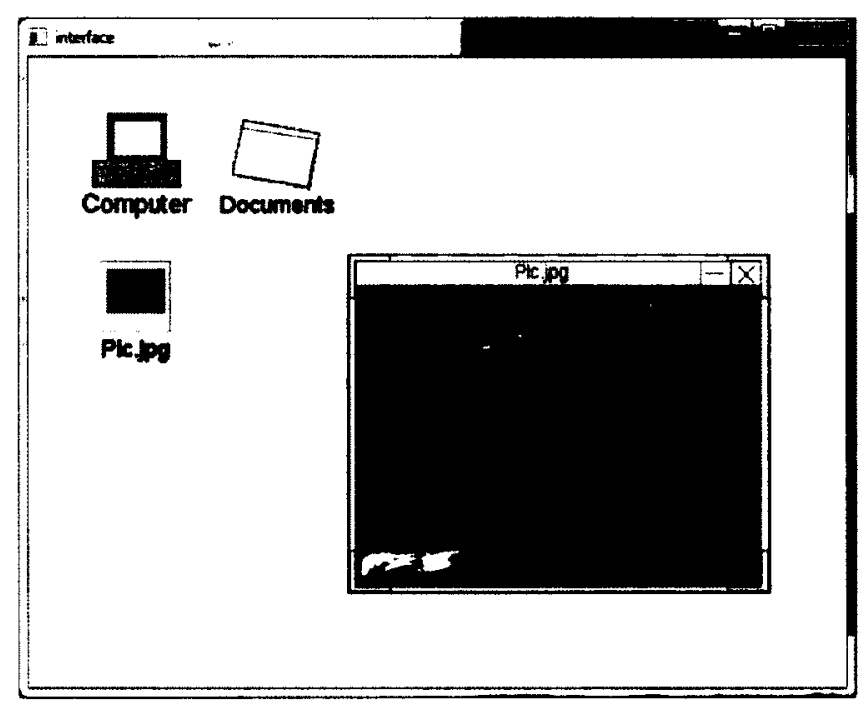

Figure 4.5. UI: Pic.jpg is open.

3. Draw a circle around the bottom-right corner of the window, and move a bit (drag), then push to release. The window "Pic.jpg" is resized.

4. Draw a circle around the title bar (name tag) of the window "Pic.jpg", and move a bit (drag), then push to release. The "Pic.jpg" window moves. 
5. Point on "X" sign of the "Pic.jpg" window, and push on it. The window "Pic.jpg" is closed.

Test Session 6: Complex task, using finger, on big-screen

\section{$\underline{\text { Task }}$}

The users are asked to include their physical actions along with the verbal definitions of each action (underlined words), e.g. tap or pinch in order for the testing person to count the number of errors in gesture recognition.

1. Wave (about five times fast waving) to start the program. The pointer appears.

2. Tap towards "Documents" icon. The "Documents" window is open.

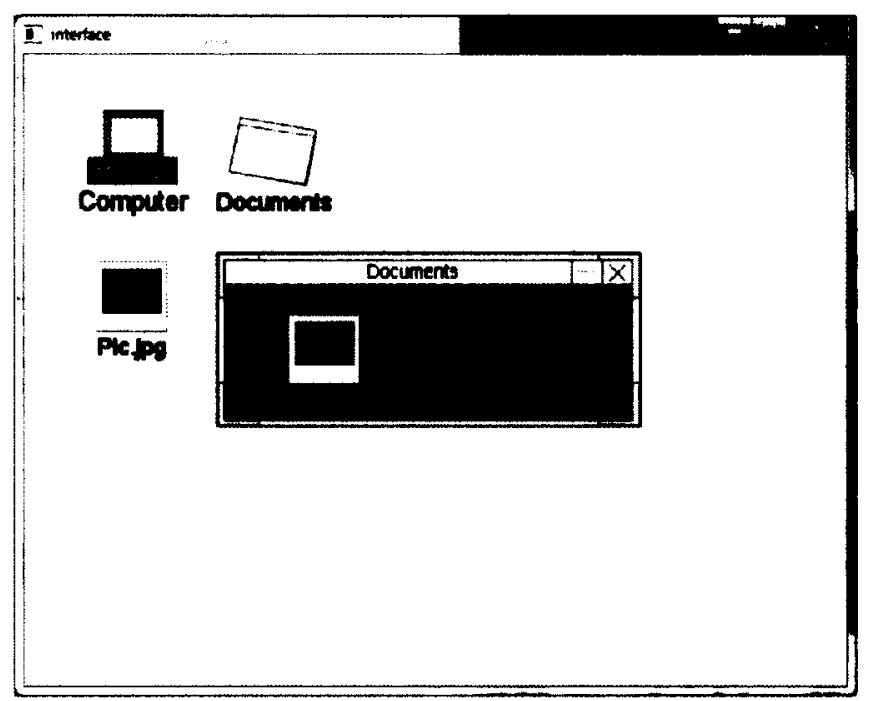

Figure 4.6. UI: Documents is open.

3. Tap towards "Pic2.jpg" icon. The "Pic2.jpg" window is open. 


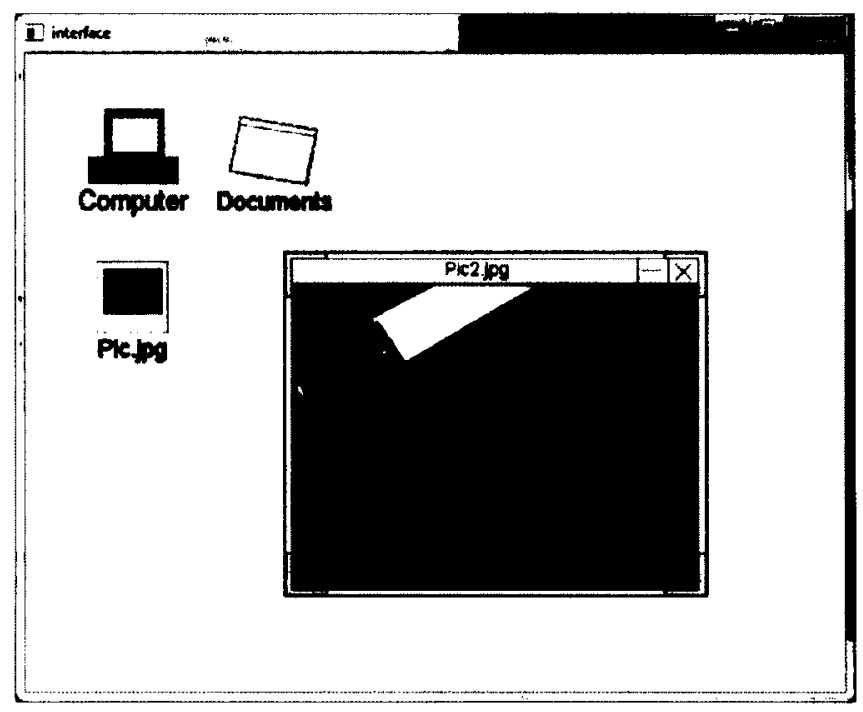

Figure 4.7. UI: Pic2.jpg is open.

4. Point on "X" sign of the "Pic2.jpg" window, and Tap towards it. The "Pic2.jpg" window is closed.

5. Point on "X" sign of the "Documents" window, and Tap towards it. The "Documents" window is closed.

6. Tap towards "Computer" icon. The "Computer" window is open.

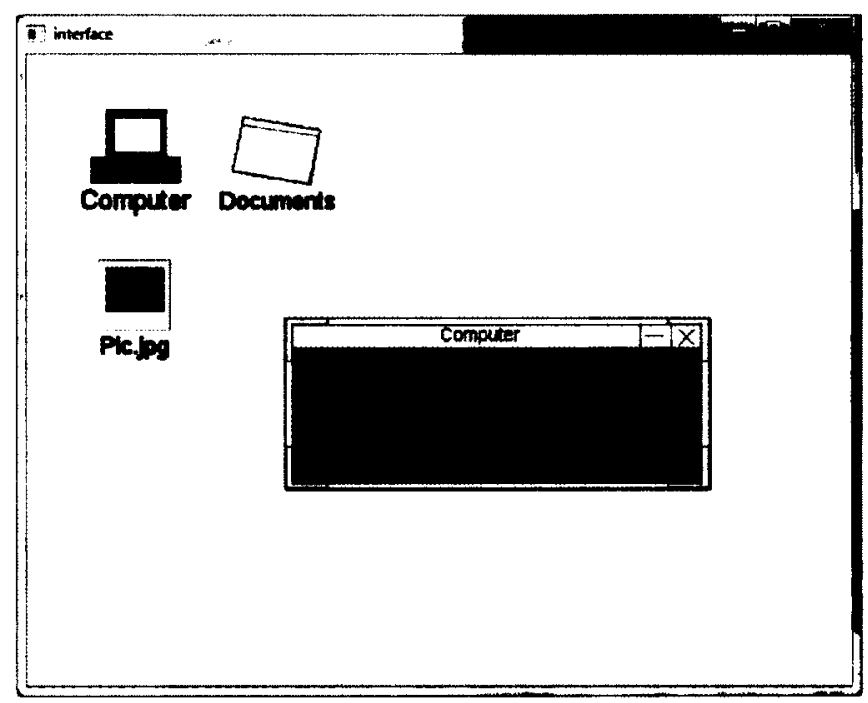

Figure 4.8. UI: Computer is open. 
7. Pinch towards the title bar (name tag) of the window "Computer", and move a bit (drag), then tap to release. The "Computer" window moves.

8. Point on "X" sign of the "Computer" window, and tap towards it. The "Computer" window is closed.

9. Pinch towards "Computer" icon, and move a bit (drag), then tap to drop it. The "Computer" icon moves.

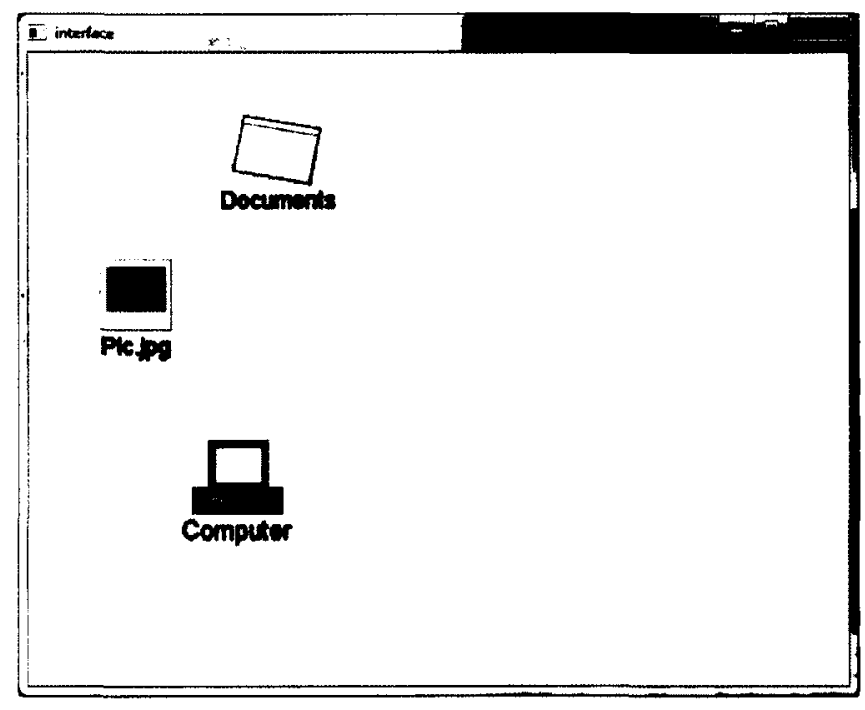

Figure 4.9. UI: Computer icon moves.

10. Press esc to finish.

\subsubsection{Questionnaire and Observation}

During the test sessions the users are requested to rate their satisfaction on a scale of 1 to 5 ( 1 for absolutely unsatisfied and 5 for extremely satisfied) on eight respective task tables (Table 4.5 and 4.6 show the samples), and to answer some extra questions on the questionnaire while the testing persons measure the observations (Table 4.7 shows a 
sample, where the number of trials for push/circle recognition are entered in the empty cells for Push\# and Circle\#, and the duration of entire task is entered in the cell for Time).

Table 4.4. Task table for Complex/Finger/Big-screen.

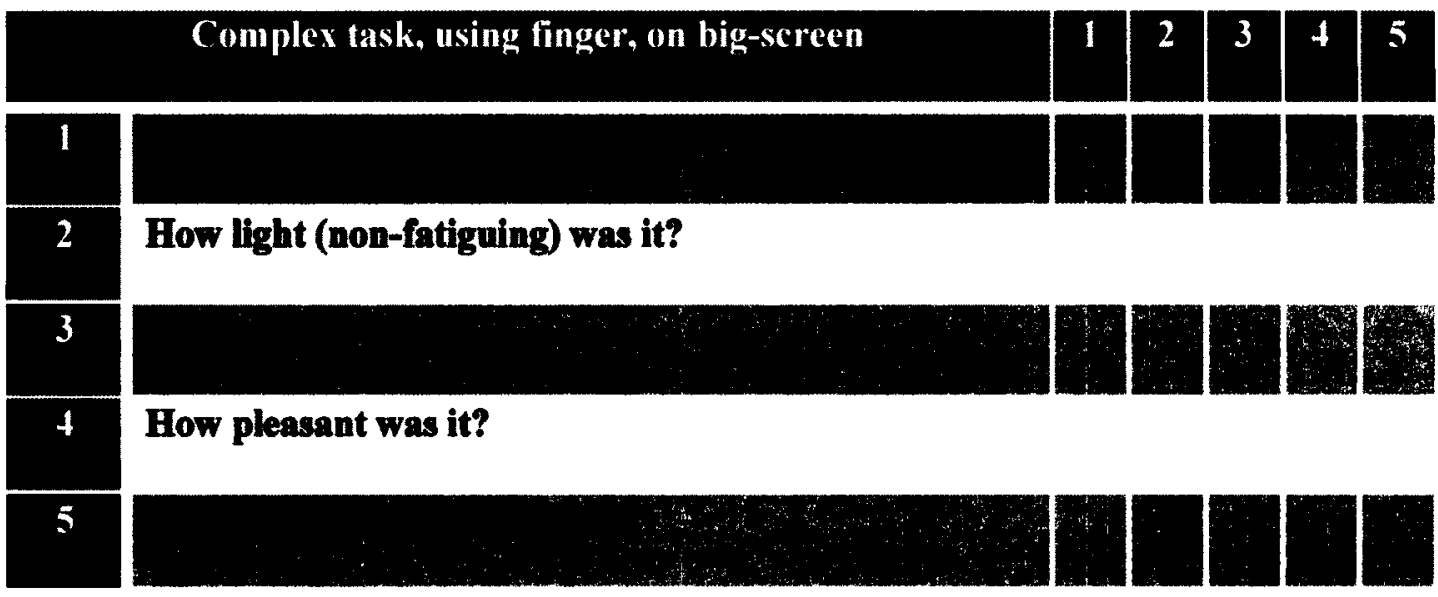

Table 4.5. Questions for four primitive tasks, here for arm and finger (the same for mouse and arm).

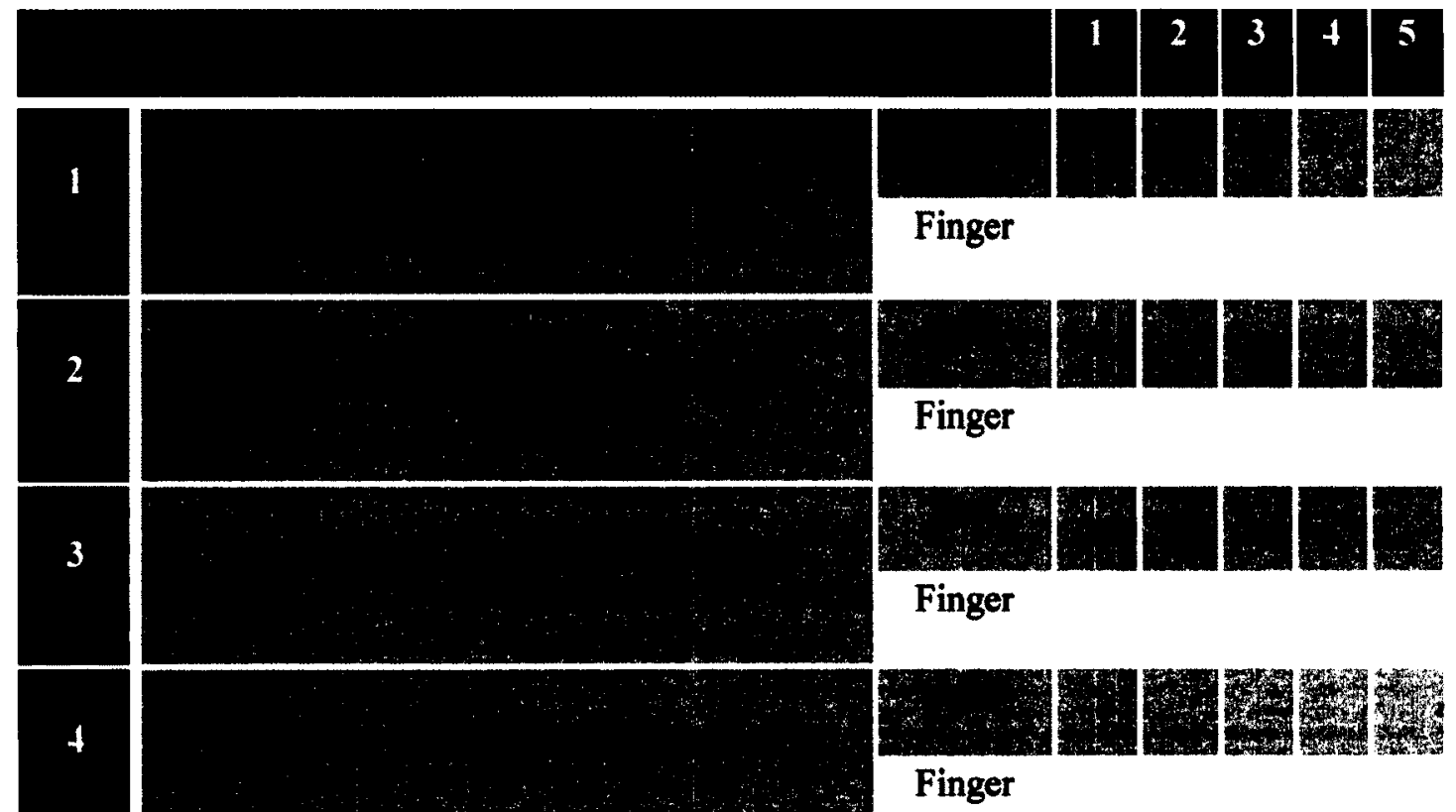


Table 4.6. Observation for Complex/Arm/Desktop.

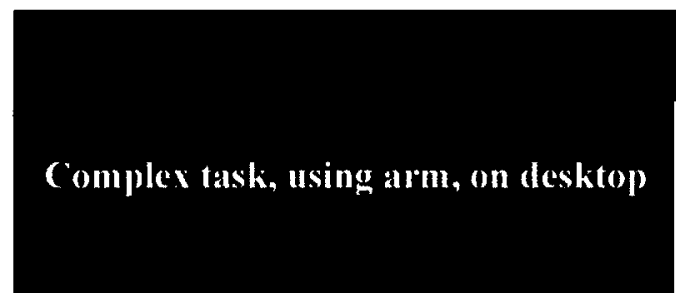

1- Program started?

2- "Documents" opened"?

3- "Pic2.jpg" opened?

4- "Pic2.jpg" closed?

5- "Documents" closed"?

6- "Computer" opened?

7. "Computer" window moved?

8- "Computer" closed?

9- "Computer" icon moved?

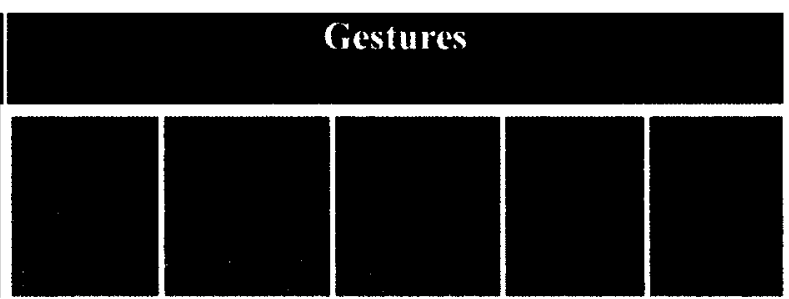

N/A N/A

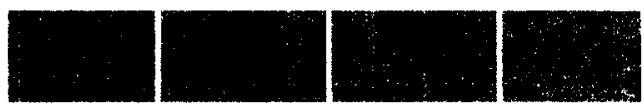

N/A N/A

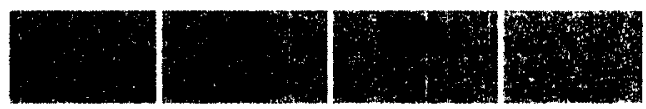

N/A N/A

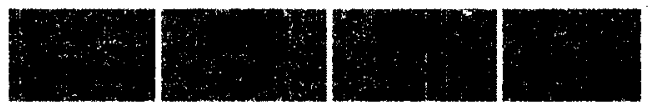

N/A

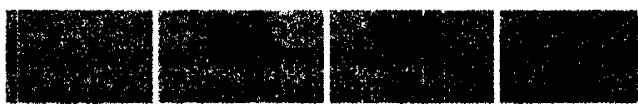

N/A

Appendix $\mathrm{C}$ shows a compressed version of the main questions answered by the users and the observation data collected by the testing persons, whereas Spatial resolution (control's accuracy) is accuracy/error rate, and Temporal resolution (control's speed) is speed rate assessed in observation process. 


\section{Chapter 5: Results and Discussions}

\subsection{Introduction}

The subjects included a mix of students and professionals. The student participants are from the Ottawa region universities and elementary school, and the professional participants are staff from the Ontario Centre of Excellence (OCE) and Ottawa hospitals. Participants were instructed to do a simple and a complex task in two different ways once using the desktop and once using the big screen. A stopwatch was used to track how much time it takes to complete each task. Through two phases (phase 1: arm gesture vs. mouse - phase 2: finger gesture vs. arm gesture), the outline of the experiment details and the methodology of our study are further elaborated in the following sections. Then we present the results and discussions, and lastly, we summarize and conclude the study with some remarks for both phases. 


\subsection{Phase 1: Arm Gestures vs. Mouse/Keyboard}

\subsubsection{Study details}

This study is conducted using 20 participants (10 males and 10 females). Nineteen participants were right-handed and one was left-handed. The researcher randomly selected 10 of the participants to do the arm gestures first and the mouse next, vice versa for the other 10 of the group. Using the desktop and the big screen was selected randomly as well. Participants include students from Carleton University and elementary school, and professionals from the OCE and Ottawa hospital. Student participants were Masters and $\mathrm{PhD}$ students from different departments including Information Technology, Computer Science, Systems and Computer Engineering, Electronics, and fifth year of an elementary school. They ranged in age from 11 to 40 years. The average age of participants was 29 years old. All participants were familiar with the use of mouse/keyboard, but have not experienced on arm gesture interface before. The participants first read the experiment instructions and were given introductions to the task they were to complete during the trial.

Twenty participants completed the trial at Interactive Media Group lab (iMG) at Carleton University. At first participants were introduced to the steps they had to follow to complete the tasks. A stopwatch registered the time taken to do each task. Each participant completed an individual 30 minute trial.

The trial was divided into two phases:

- Training phase

- Test phase and satisfaction phase to complete a paper questionnaire 


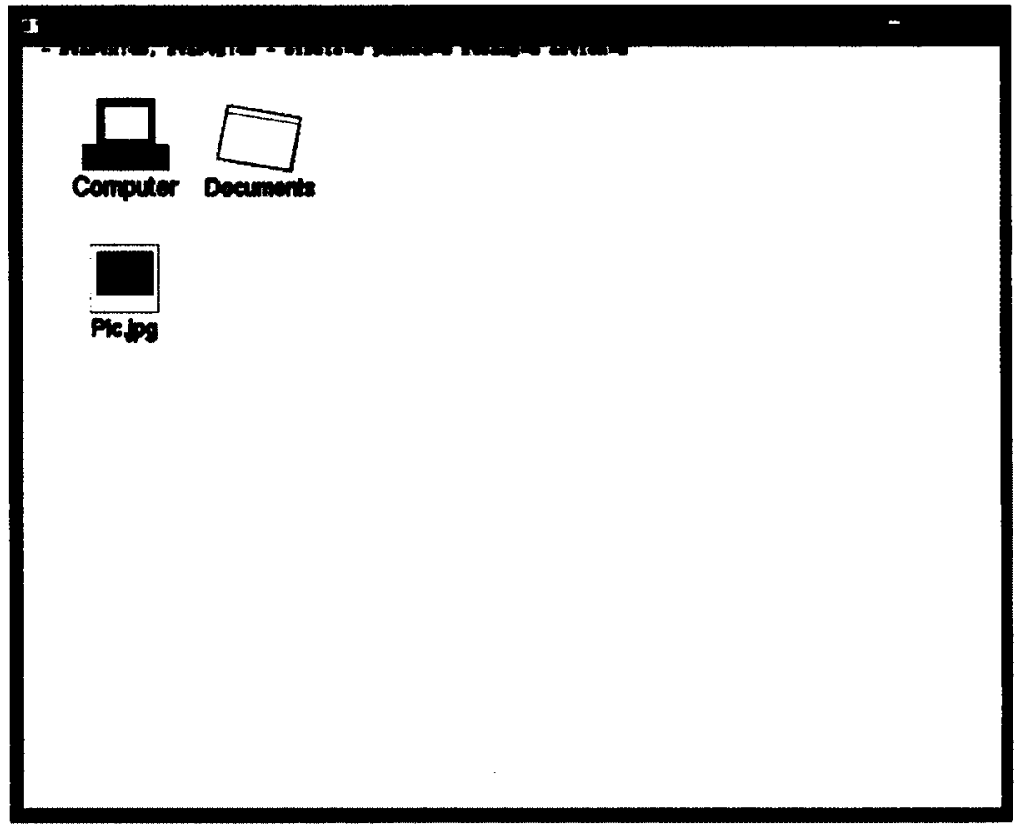

Figure 5.1. Interface.

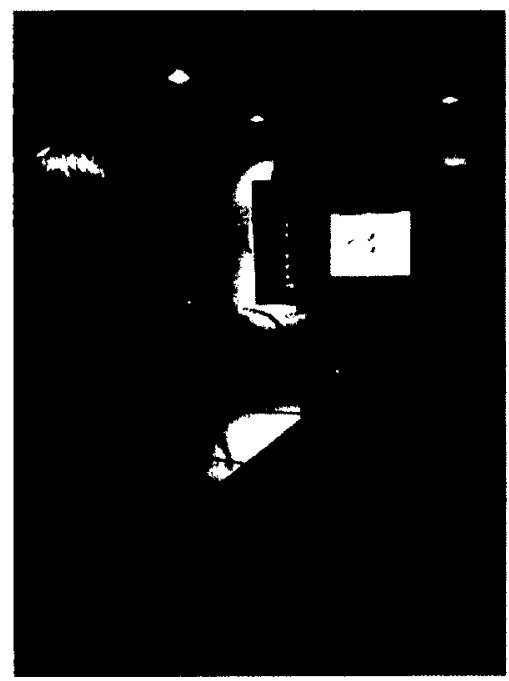

Figure 5.2. A participant is interacting with the big screen using arm gesture.

In the Satisfaction phase, participants were asked to complete a paper questionnaire. The aim of the questionnaire was to get the opinions of the group on using the two test methods and what they perceived as difficulties while completing the task. 


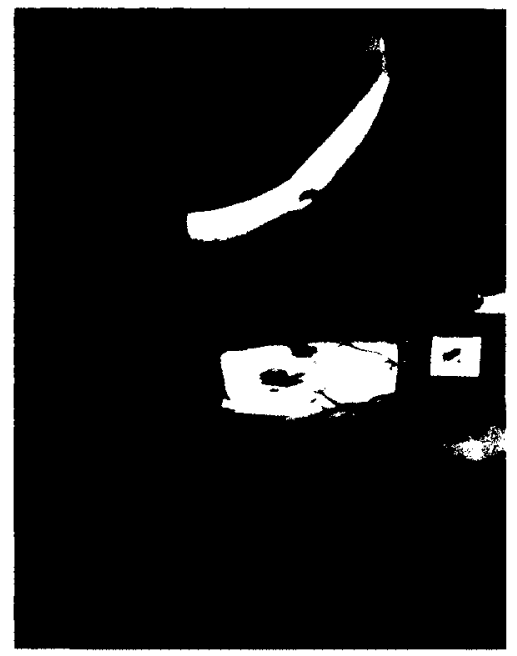

Figure 5.3. A participant is interacting with the desktop using arm gesture.

\subsubsection{Results and Evaluation}

\subsubsection{Hypotheses and Statistical Analyses}

For the different factors being studied, 3-way repeated analysis of variances (ANOVA) is carried out for three independent variables:

1- Difficulty (simple task vs. complex task)

2- Input device (mouse vs. arm gestures)

3- Output device (desktop vs. big-screen)

All analysis are concluded at $p<0.05$ significance level and for 20 participants. Our ANOVA analysis is accompanied by an extra t-test analysis particularly for naturalness and fatigue. This redundancy is carried out in order to confirm our multi-factor analysis with a single-factor analysis. The results of the t-test support the ANOVA analysis. 
Time:

One researcher was taking the time with a stopwatch and one researcher counting the errors.

Table 5.1. Task duration.

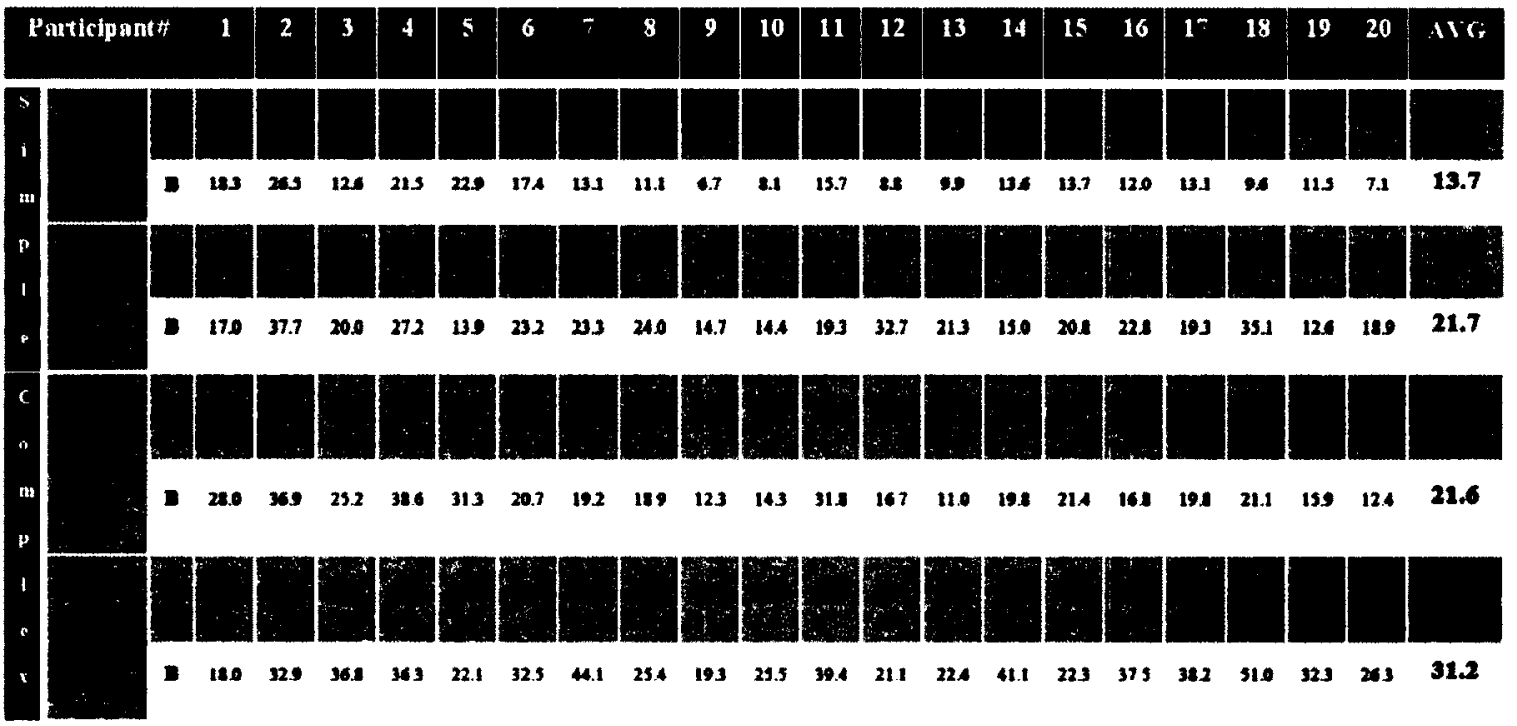

Table 5.1 shows the times taken to complete the simple task and the complex task once using arm gesture and once with the mouse/keyboard.

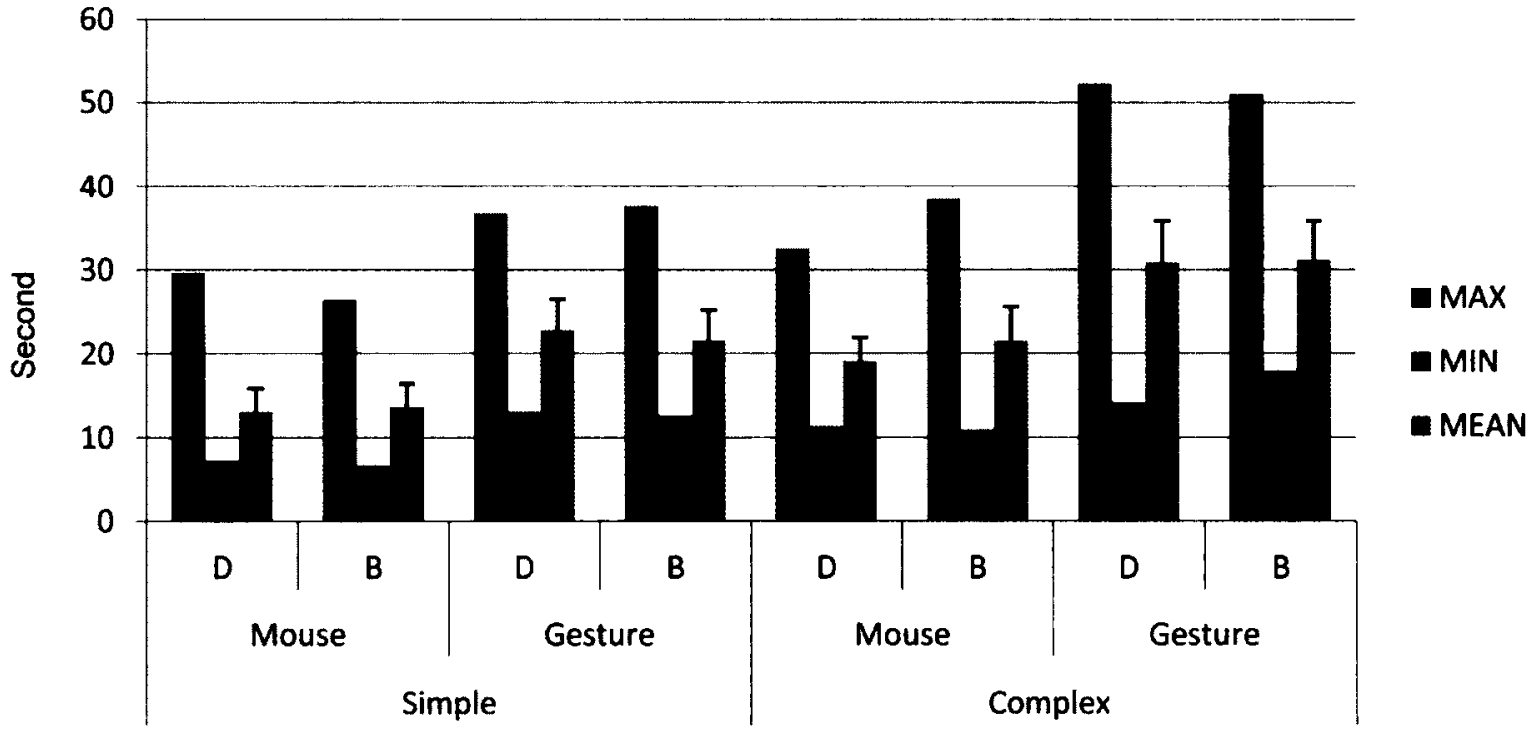

Figure 5.4. Temporal MAX/MIN/MEAN/ST DEV facts $(D=d e s k t o p, B=b i g-s c r e e n)$. 


\section{Hypothesis- using a mouse is faster than using arm gestures as inputs.}

The analysis illustrates that for variable $1, \mathrm{~F}(1,2504.306)=66.994, \mathrm{P}=0.0000\left(\mathrm{M}_{\text {simple }}=\right.$ $17.83, \mathrm{SD}_{\text {simple }}=7.67$ vs. $\mathrm{M}_{\text {complex }}=25.74, \mathrm{SD}_{\text {complex }}=9.80$ ). This illustrates that task complexity has significant effect on time. This effect is as expected since the two tasks were initially designed to illustrate different difficulty levels for using the system. For variable $2, \mathrm{~F}(1,3820.070)=41.163, \mathrm{P}=0.0000\left(\mathrm{M}_{\text {mouse }}=16.90, \mathrm{SD}_{\text {mouse }}=7.0868 \mathrm{vs}\right.$. $\mathrm{M}_{\text {gesture }}=26.67, \mathrm{SD}_{\text {gesture }}=9.3749$ ), which implies that using gestures also has significant effect on time. For variable $3, F(1,10.404)=0.646, P=0.4316$ which illustrates that the screen type does not have a significant effect on time. Moreover, the analysis shows no significant effect on time for variables 1 and 2 combined $F(1,29.929)=1.371, P=$ 0.2562 , variables 1 and 3 combined $F(1,28.392)=1.641, P=0.2156$, and finally variables 2 and 3 combined, $F(1,37.056)=1.131, P=0.3008$. Combination of the three variables $(1,2$, and 3$) F(1,0.121)=0.006, P=0.9370$ also do not show any significant effect on time. Based on the above, the initial hypothesis is confirmed meaning gesture inputs are significantly slower than using a mouse.

\section{Easiness:}

Hypothesis- Using arm gestures as inputs is easier than mouse.

Analyzing the feedback from participants regarding easiness of experiments given the 3 variables defined earlier shows that the only significant effect is caused by variable 2 , $\mathrm{F}(1,19.600)=23.059, \mathrm{P}=0.0001\left(\mathrm{M}_{\text {mouse }}=4.3750, \mathrm{SD}_{\text {mouse }}=0.8325\right.$ vs. $\mathrm{M}_{\text {gesture }}=$ 3.6750, $\mathrm{SD}_{\text {gesture }}=0.9517$ ). This means that according to participants, the only variable with significant effect on easiness is the input device (mouse vs. gesture). For variable 1, 
$\mathrm{F}(1,0.100)=0.134, \mathrm{P}=0.7181$ and for variable $3, \mathrm{~F}(1,1.225)=2.730, \mathrm{P}=0.1149$. For combination of variables 1 and $2, F(1,0.100)=0.409, P=0.5303$, variables 1 and 3 , $\mathrm{F}(1,0.225)=0.371, \mathrm{P}=0.5497$, variables 2 and $3, \mathrm{~F}(1,4.225)=4.219, \mathrm{P}=0.0540$, and finally for variables 1,2 , and $3, F(1,0.225)=0.609, P=0.4449$ which indicates that there is no significant effect. According to the provided statistics, the initial hypothesis is rejected which indicates that using a mouse is significantly easier than using arm gestures.

\section{Fatigue:}

Hypothesis- Using arm gestures produces more fatigue compared to mouse.

In this experiment the participants have been asked to rank higher if more fatigue is experienced. The feedback obtained from participants indicates that similar to easiness, variable 2 is the only one with significant effect $F(1,45.156)=31.813, P=0.0000\left(M_{\text {mouse }}\right.$ $=1.4000, \mathrm{SD}_{\text {mouse }}=0.7730$ vs. $\left.\mathrm{M}_{\text {gesture }}=2.4625, \mathrm{SD}_{\text {gesture }}=0.9929\right)$. This indicates that the input device is the only determining parameter in fatigue. For variable $1, \mathrm{~F}(1,1.406)=$ $3.065, \mathrm{P}=0.0961$ and for variable $3, \mathrm{~F}(1,0.506)=1.351, \mathrm{P}=0.2595$ respectively. For combination of variables 1 and $2, F(1,0.006)=0.015, P=0.9050$, variables 1 and 3 , $\mathrm{F}(1,0.006)=0.018, \mathrm{P}=0.8949$, variables 2 and $3, \mathrm{~F}(1,0.756)=0.657, \mathrm{P}=0.4276$, and finally variables 1,2 , and $3, F(1,0.756)=1.322, P=0.2645$. Based on the above mentioned figures, the initial hypothesis is approved, meaning arms gestures significantly causes more fatigue compared to using a mouse. 
Table 5.2. Fatigue for simple task using desktop and results of t-test.

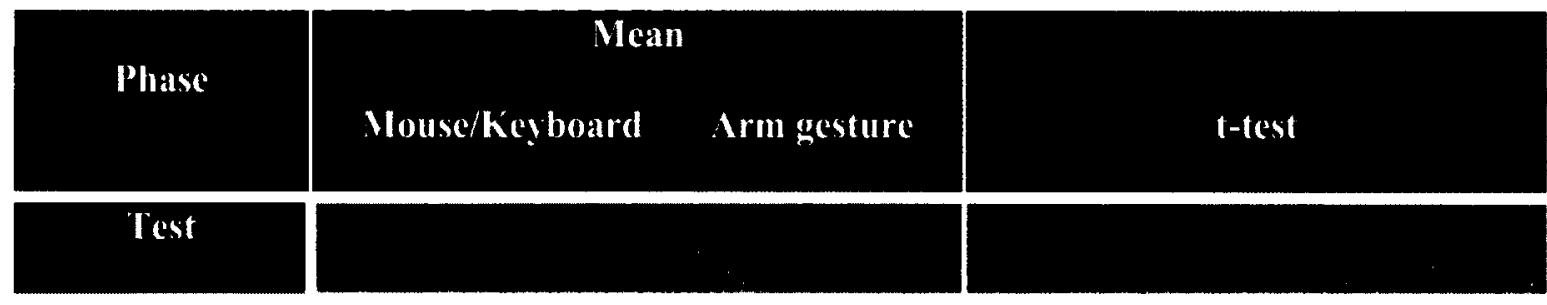

Table 5.3. Fatigue for simple task using big-screen and results of t-test.

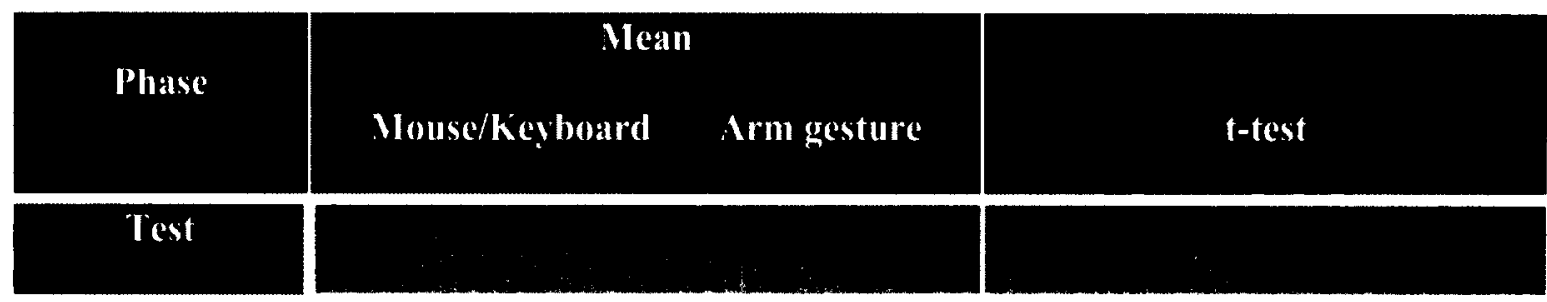

Table 5.4. Fatigue for complex task using desktop and results of t-test.

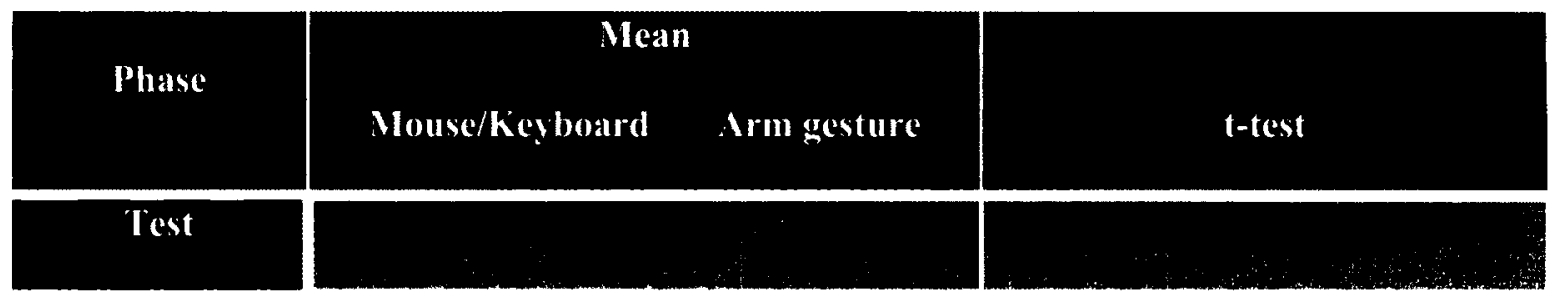

Table 5.5. Fatigue for complex task using big-screen and results of t-test.

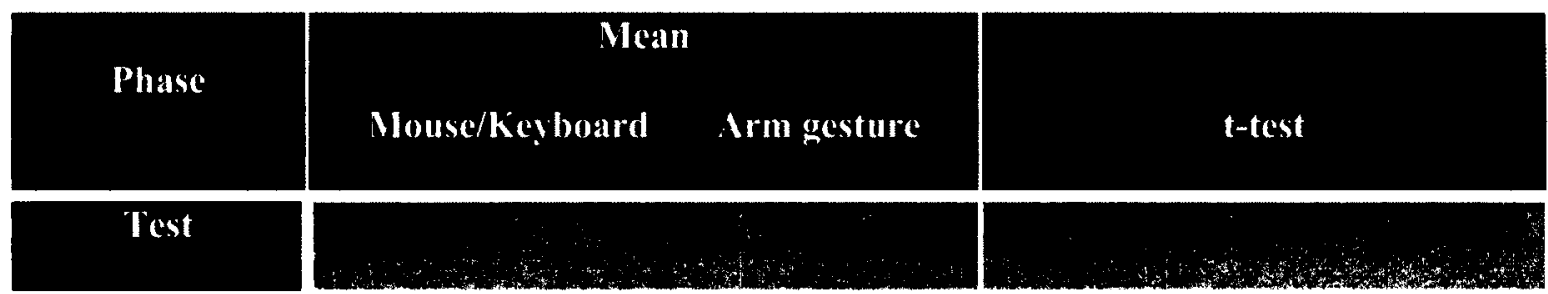




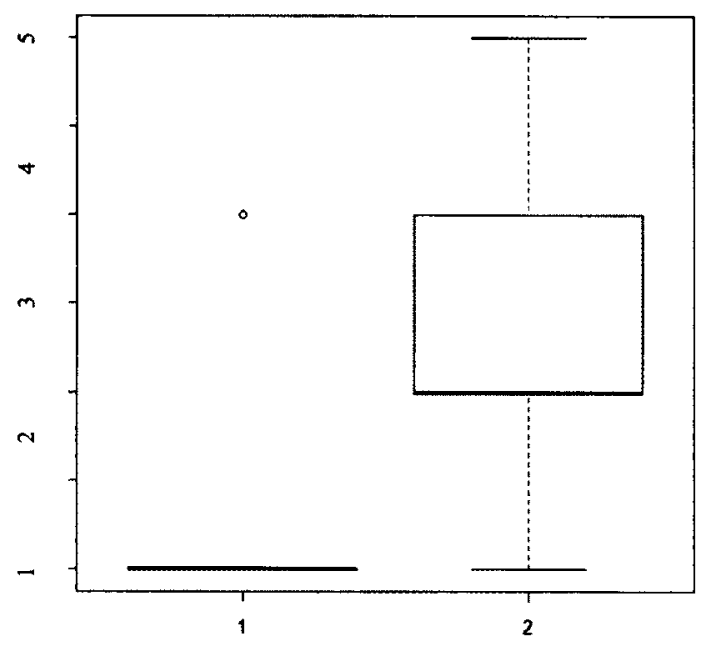

(a)

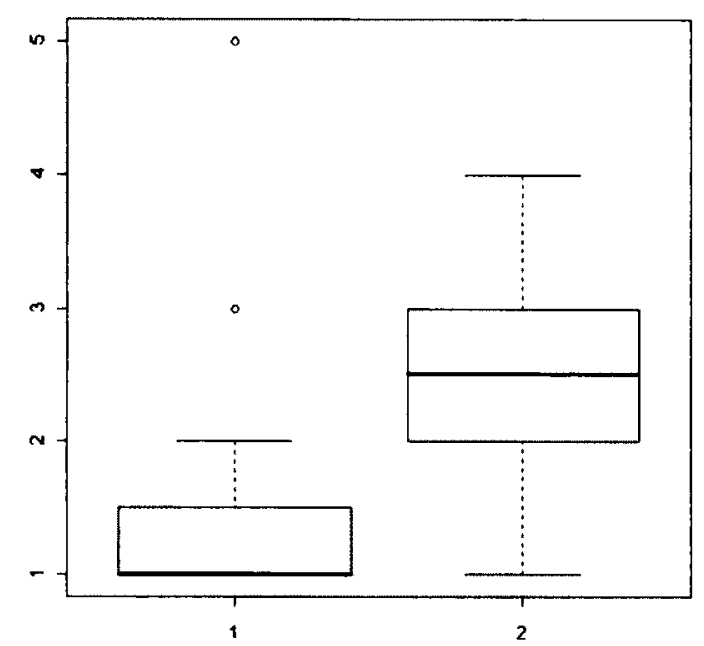

(c)

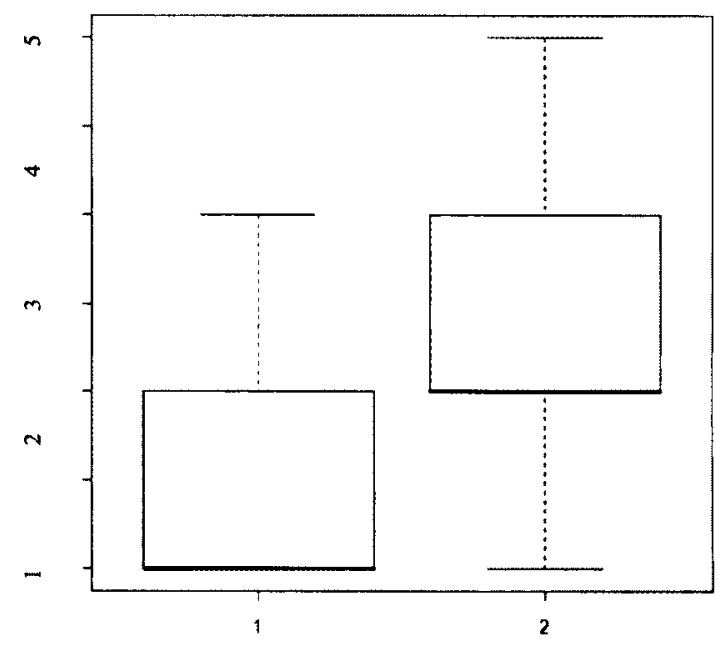

(b)

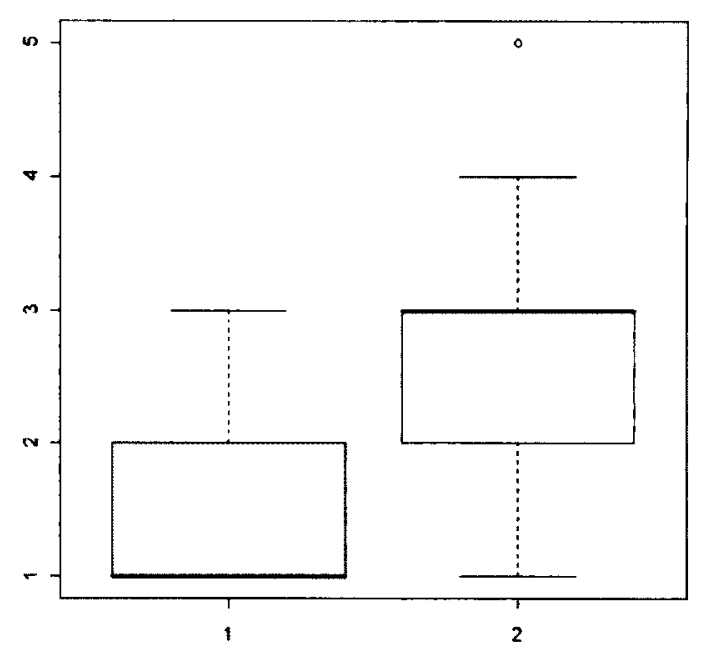

(d)

Figure 5.5. Mean and SD of fatigue comparing 1- mouse/keyboard and 2- arm gesture using a) desktop for simple task, b) big-screen for simple task, c) desktop for complex task, and d) big-screen for complex task. The dots on the boxplots represent the outliers. 


\section{Naturalness:}

\section{Hypothesis- Using arm gestures is more natural than using a mouse.}

For this factor, none of the variables shows any significant effect. The calculated statistical values for variable $1, F(1,0.000)=0.000, P=1.0000$, for variable 2 , $\mathrm{F}(1,10.000)=4.153, \mathrm{P}=0.0557$, and for variable $3, \mathrm{~F}(1,0.225)=0.851, \mathrm{P}=0.3679$. These results indicate that variables 1,2 , and 3 do not have any significant impact on naturalness of tasks. However, combination of variables 2 and 3 show significant effect $\mathrm{F}(1,5.625)=6.628, \mathrm{P}=0.0186\left(\mathrm{M}_{\text {mouse-desktop }}=3.4500, \mathrm{SD}_{\text {mouse-desktop }}=1.1082\right.$, vs. $\mathrm{M}_{\text {mouse- }}$ bigscreen $=3, \mathrm{SD}_{\text {mouse-bigscreen }}=1.1983$, vs. $\mathrm{M}_{\text {gesture-desktop }}=3.5750, \mathrm{SD}_{\text {gesture-desktop }}=0.9306$, vs. $\mathrm{M}_{\text {gesture-bigscreen }}=3.8750, \mathrm{SD}_{\text {gesture-bigscreen }}=0.8530$ ). This means that the input device when combined with a particular output device will show significant effect on naturalness. Multiple one-way ANOVAs further indicate that mouse when used on desktop is significantly more natural than mouse used on big-screen. Moreover, gestures used on big-screen are significantly more natural than mouse used on both desktop and big-screen. Combination of variables 1 and $2, F(1,0.400)=0.910, P=0.3520$, variables 1 and $3, F(1,0.225)=0.533, P=0.4744$, and finally variables 1,2 , and $3, F(1,0.625)=$ $1.067, \mathrm{P}=0.3145$, show no significant effect. According to the above mentioned figures, the hypothesis is rejected, meaning arm gestures as inputs do not feel significantly more natural compared to mouse. However, it is shown that using arm gestures on big-screen is significantly more natural than using a mouse on both the desktop and the big-screen. 
Table 5.6. Naturalness for simple task using desktop and results of t-test.

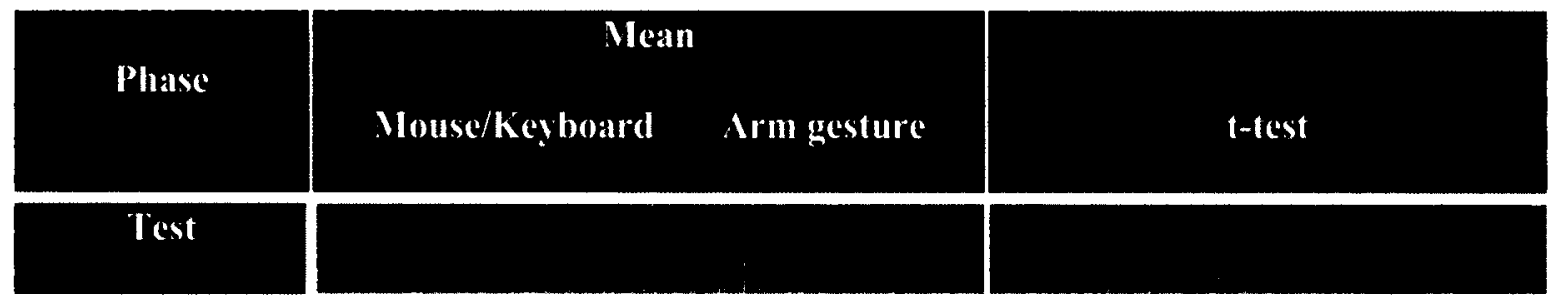

Table 5.7. Naturalness for simple task using big-screen and results of t-test.

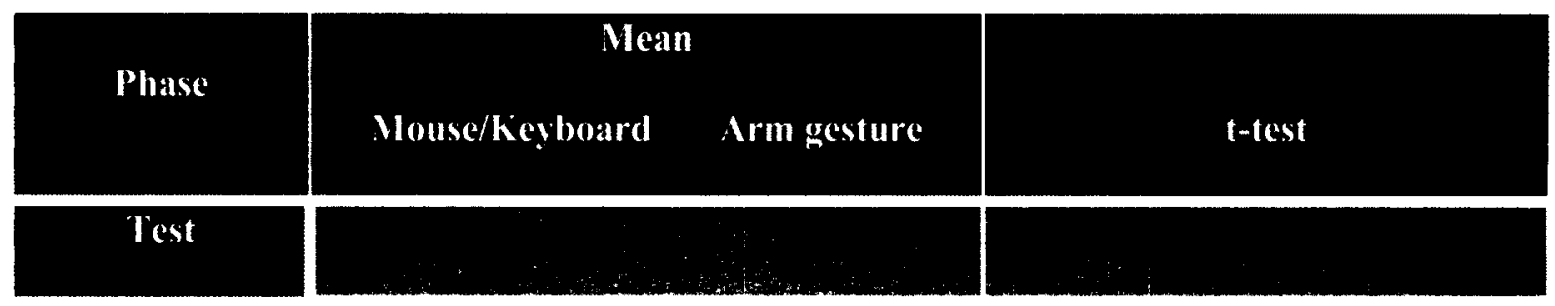

Table 5.8. Naturalness for complex task using desktop and results of t-test.
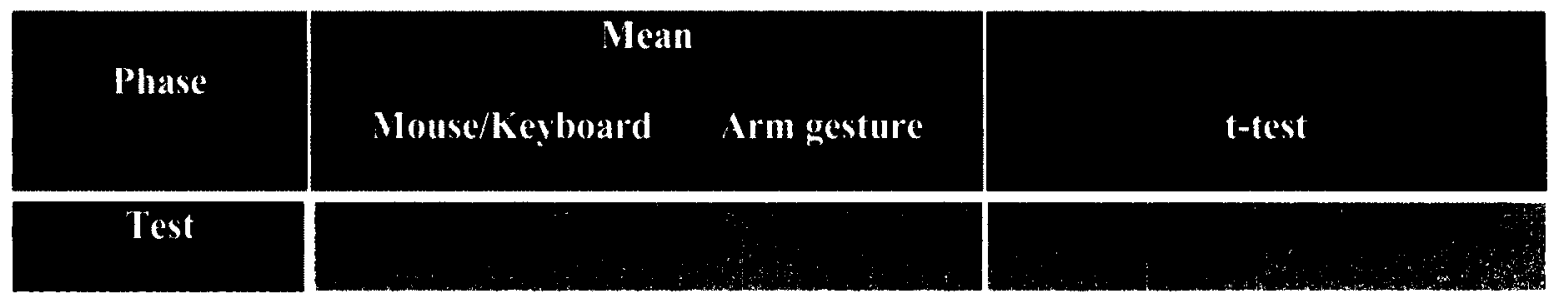

Table 5.9. Naturalness for complex task using big-screen and results of t-test.

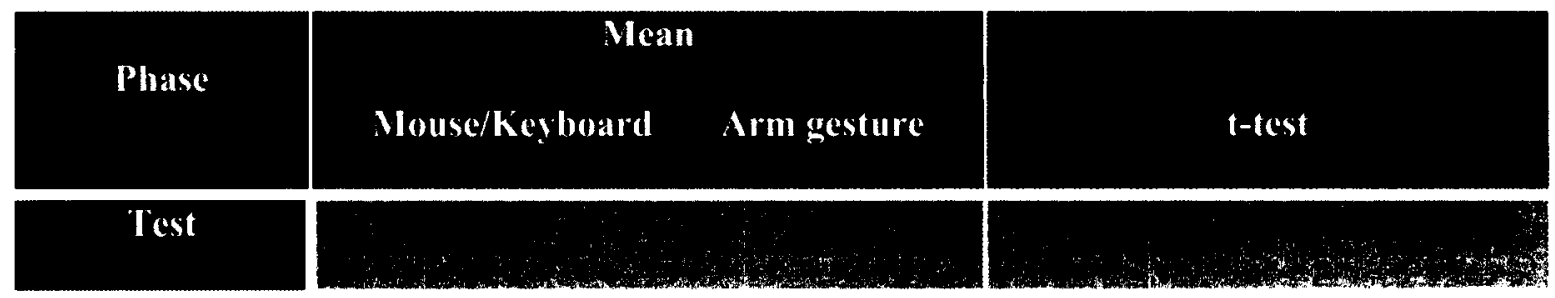




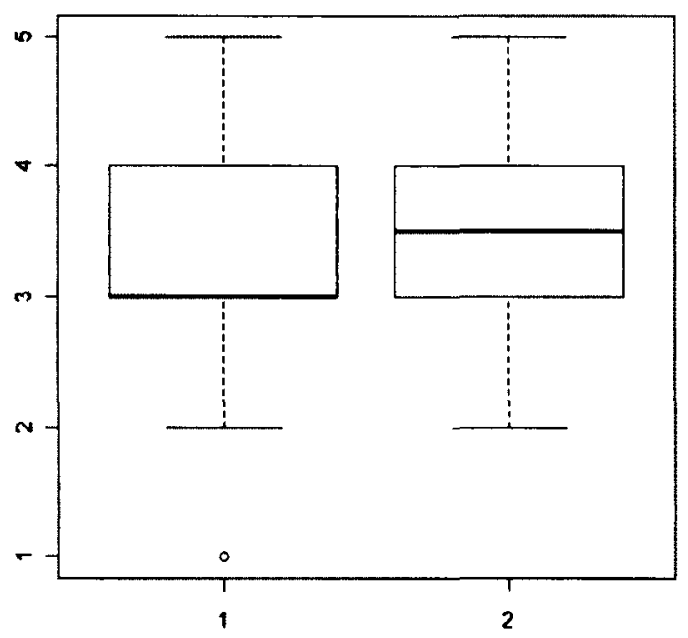

(a)

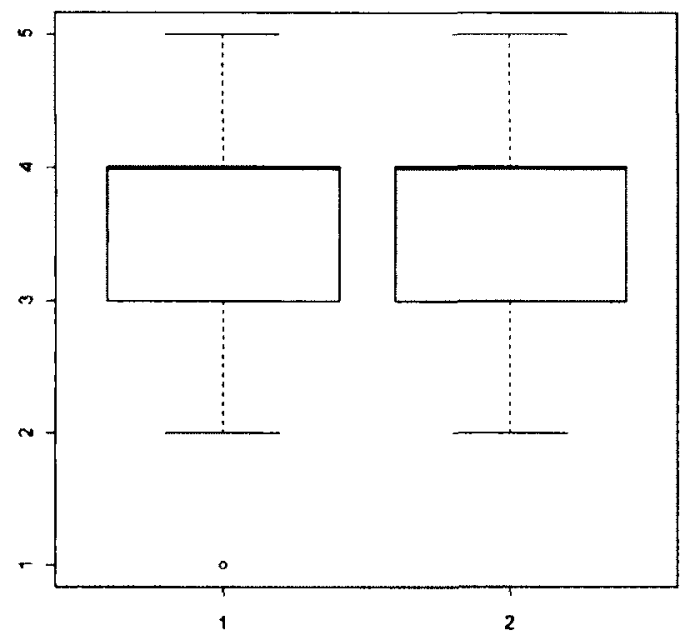

(c)

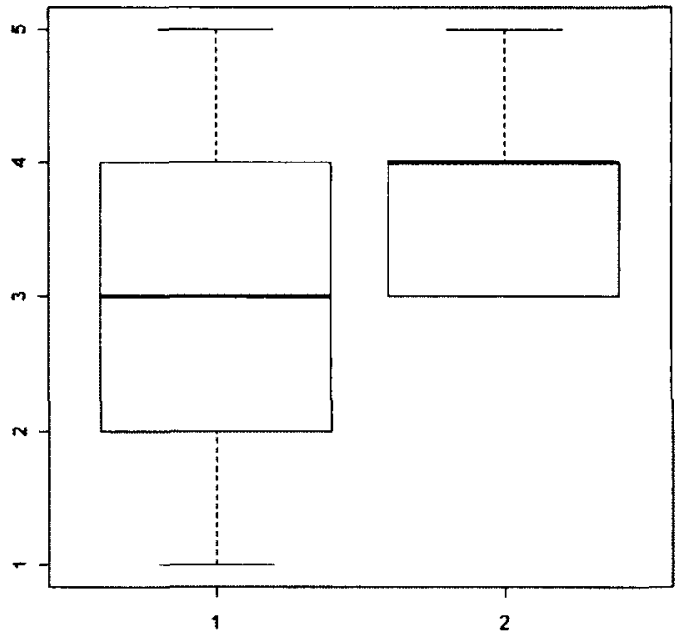

(b)

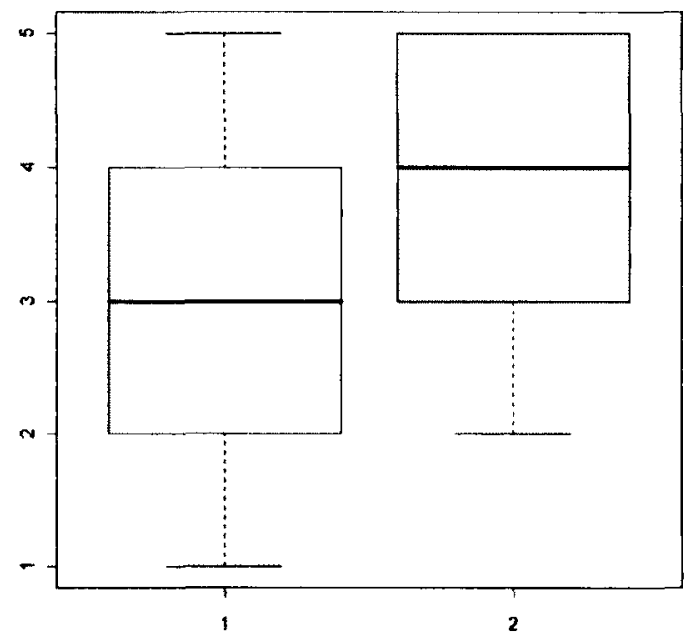

(d)

Figure 5.6. Mean and SD of naturalness comparing 1- mouse/keyboard and 2- arm gesture using a) desktop for simple task, b) big-screen for simple task, c) desktop for complex task, and d) big-screen for complex task. The dots on the boxplots represent the outliers. 


\section{Pleasantness:}

\section{Hypothesis- Using arm gestures as inputs is more pleasant than using mouse.}

When analyzing the participant feedback for pleasantness, a similar trend to that of naturalness is observed. Variable $1, F(1,0.006)=0.016, P=0.9020$, variable 2 , $\mathrm{F}(1,6.806)=3.824, \mathrm{P}=0.0654$, and variable $3, \mathrm{~F}(1,0.506)=1.351, \mathrm{P}=0.2595$ show no significant effect. Combination of variables 1 and $2, F(1,1.056)=3.055, P=0.0966$, variables 1 and $3, \mathrm{~F}(1,0.306)=1.347, \mathrm{P}=0.2601$, and variables 1,2 , and $3, \mathrm{~F}(1,0.506)=$ $1.572, \mathrm{P}=0.2251$ show no significant effect as well. Similar to naturalness, the only set of variables which illustrate an effect are combination of factors 2 and $3, F(1,8.556)=$ $7.716, \mathrm{P}=0.0120\left(\mathrm{M}_{\text {mouse-desktop }}=3.7250, \mathrm{SD}_{\text {mouse-desktop }}=0.9868\right.$ vs. $\mathrm{M}_{\text {mouse-bigscreen }}=$ $3.1500, \mathrm{SD}_{\text {mouse-bigscreen }}=1.0266$, vs. $\mathrm{M}_{\text {gesture-desktop }}=3.6750, \mathrm{SD}_{\text {gesture-desktop }}=0.8590$, vs. $\mathrm{M}_{\text {gesture-bigscreen }}=4.0250, \mathrm{SD}_{\text {gesture-bigscreen }}=0.8317$ ). Therefore there is significant interaction between input and output device when pleasantness is being analyzed. Multiple one-way ANOVAs further indicate that mouse when used on desktop is significantly more pleasant than mouse used on big-screen. Furthermore, arm gestures used on big-screen is significantly more pleasant than mouse used on desktop, mouse used on big-screen, and arm gestures used on desktop. Based on these results, similar to naturalness, the initial hypothesis is rejected. But again, it is revealed that the hypothesis does hold true on big-screens, meaning using arm gestures is significantly more pleasant than mouse when performed on big-screens. Also it is shown that arm gestures used on big-screen is significantly more pleasant compared to when it is used on desktop. 


\section{Overall Satisfaction:}

Hypothesis- Overall, using arm gestures as inputs is a more popular experience compared to mouse.

In the overall ranking obtained from participants, no particular variable shows significant effect. This can be due to the fact that while some parameters such as naturalness are ranked higher for gesture on the big-screen, the fatigue level is increased at the same time. This experience, we believe leads to an overall insignificant ranking. The calculated values are as follows: For variable $1, \mathrm{~F}(1,0.006)=0.019, \mathrm{P}=0.8928$, for variable 2 , $F(1,0.306)=0.341, P=0.5662$, and for variable $3, F(1,0.306)=0.721, P=0.4063$. Similarly for combination of variables, no effect is observed since for variables 1 and 2 , $F(1,0.156)=0.704, P=0.4120$, variables 1 and $3, F(1,0.006)=0.022, P=0.8833$, variables 2 and $3, F(1,3.906)=4.249, P=0.0532$, and finally for all three variables 1,2 , and $3, \mathrm{~F}(1,0.006)=0.035, \mathrm{P}=0.8531$. Based on this analysis, the hypothesis is rejected, meaning neither input hold a significant popularity over the other.

\subsubsection{Number of Errors}

The following tables show the average number of trials before all participants successfully perform a task (average number of errors in each mouse/gesture task for all 20 users). 
Table 5.10. Observation for simple task using mouse on desktop.

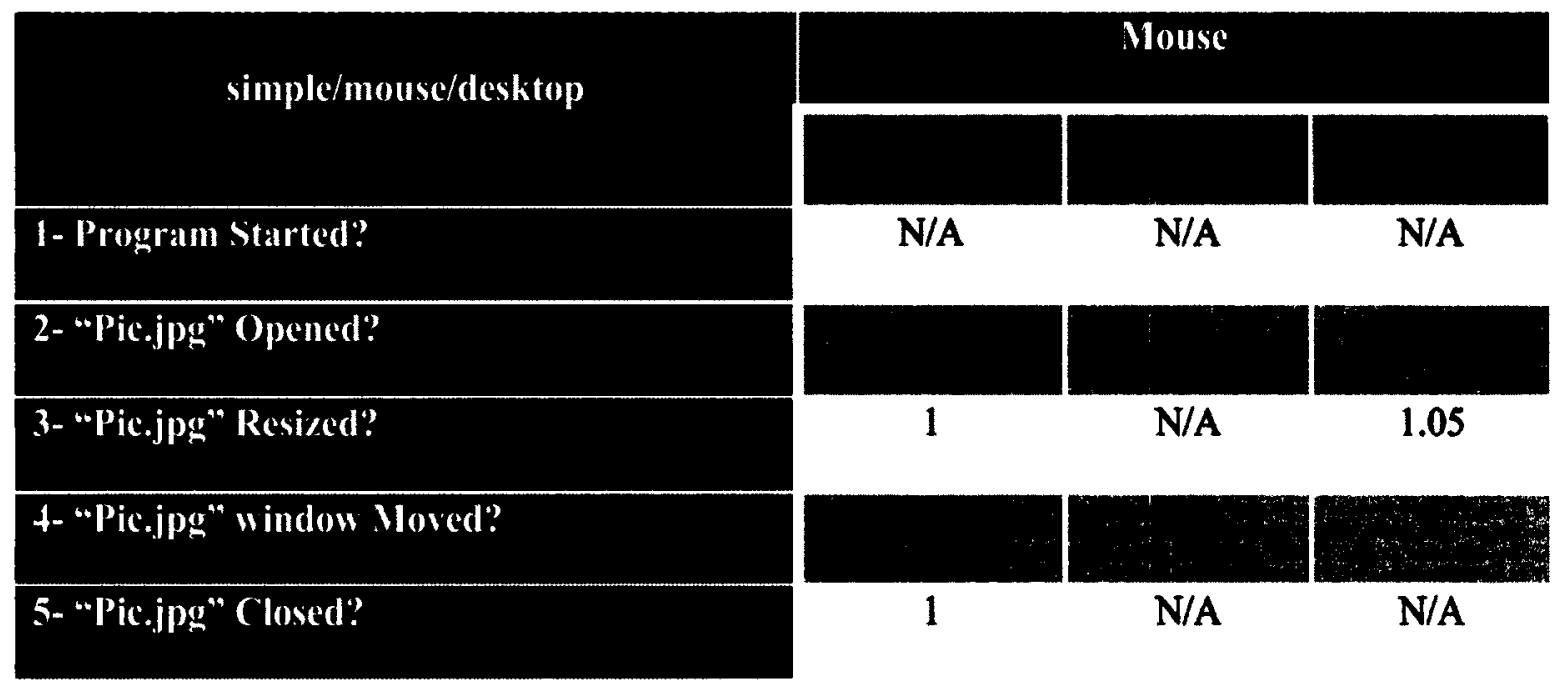

Table 5.11. Observation for simple task using mouse on big-screen.

\begin{tabular}{|l|}
\hline \multicolumn{1}{|c|}{ simple/mouse/hig-screen } \\
\hline 1- Program Started? \\
\hline 2- "Pic.jpg" (Opened?' \\
\hline 3- "Pic.jpg" Resized? \\
\hline 4- "Pic.jpg" window Noved? \\
\hline 5- "Pic.jpg" ( losed? \\
\hline
\end{tabular}

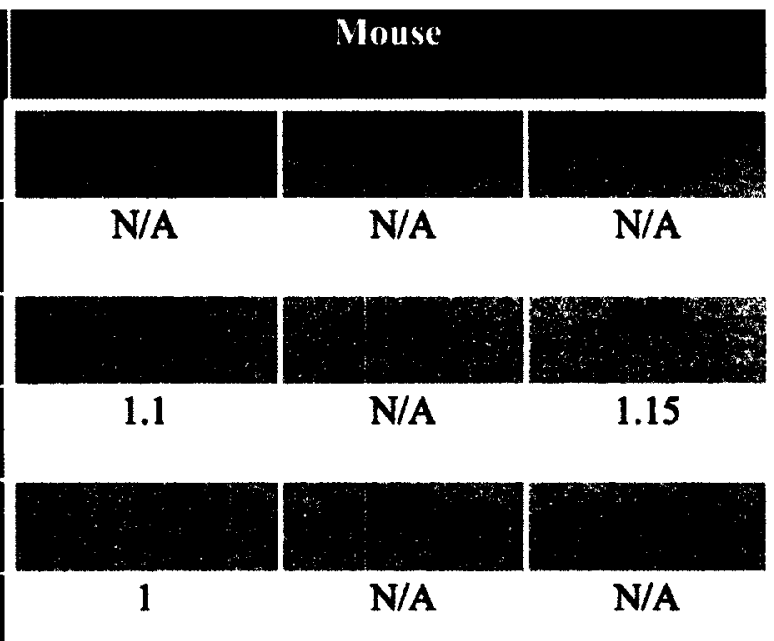


Table 5.12. Observation for simple task using gesture on desktop.
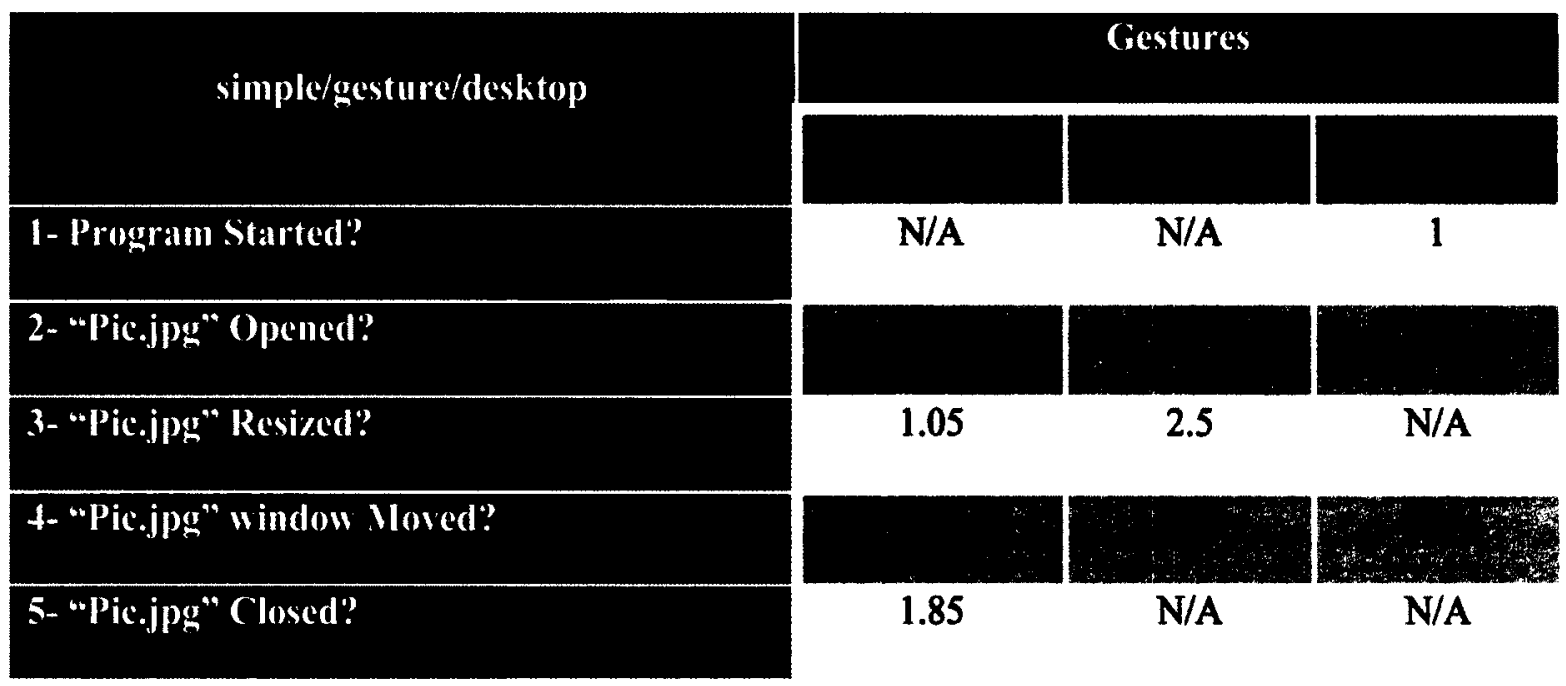

Table 5.13. Observation for simple task using gesture on big-screen.
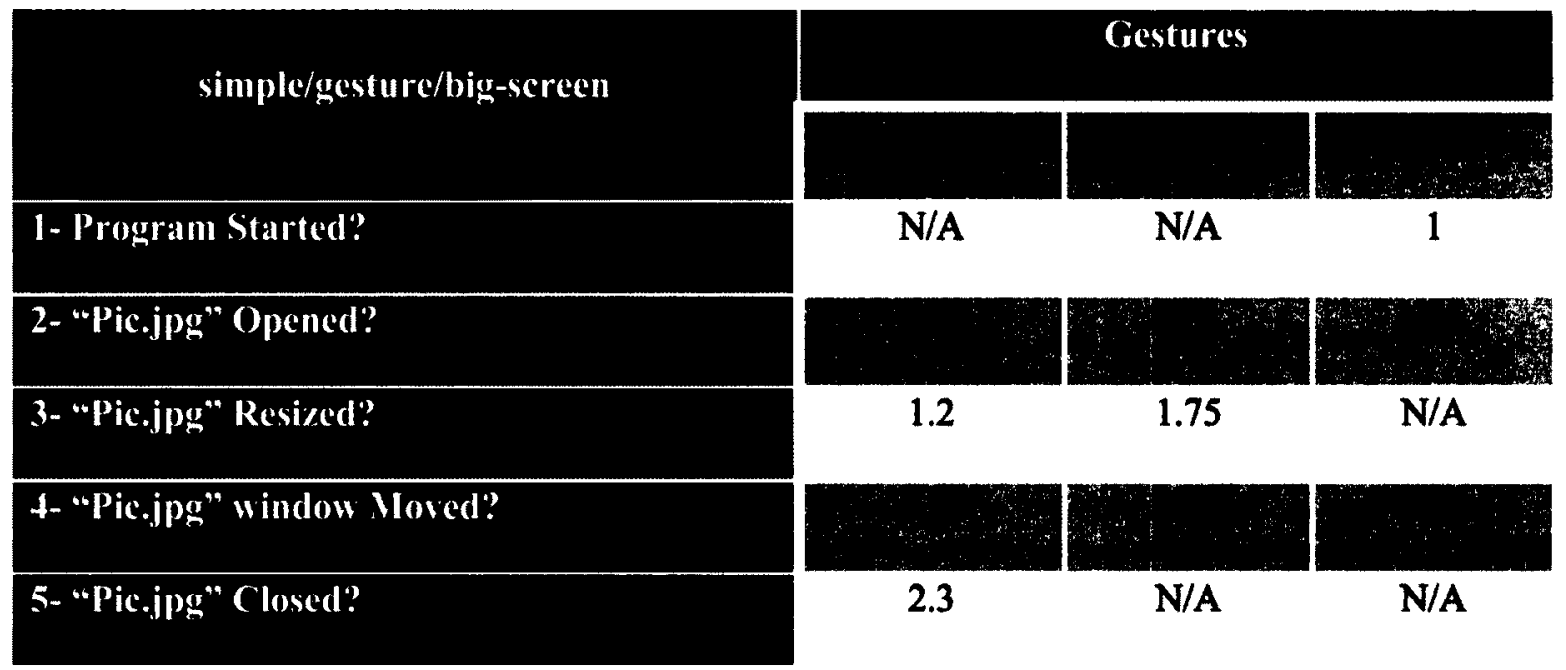
Table 5.14. Observation for complex task using mouse on desktop.

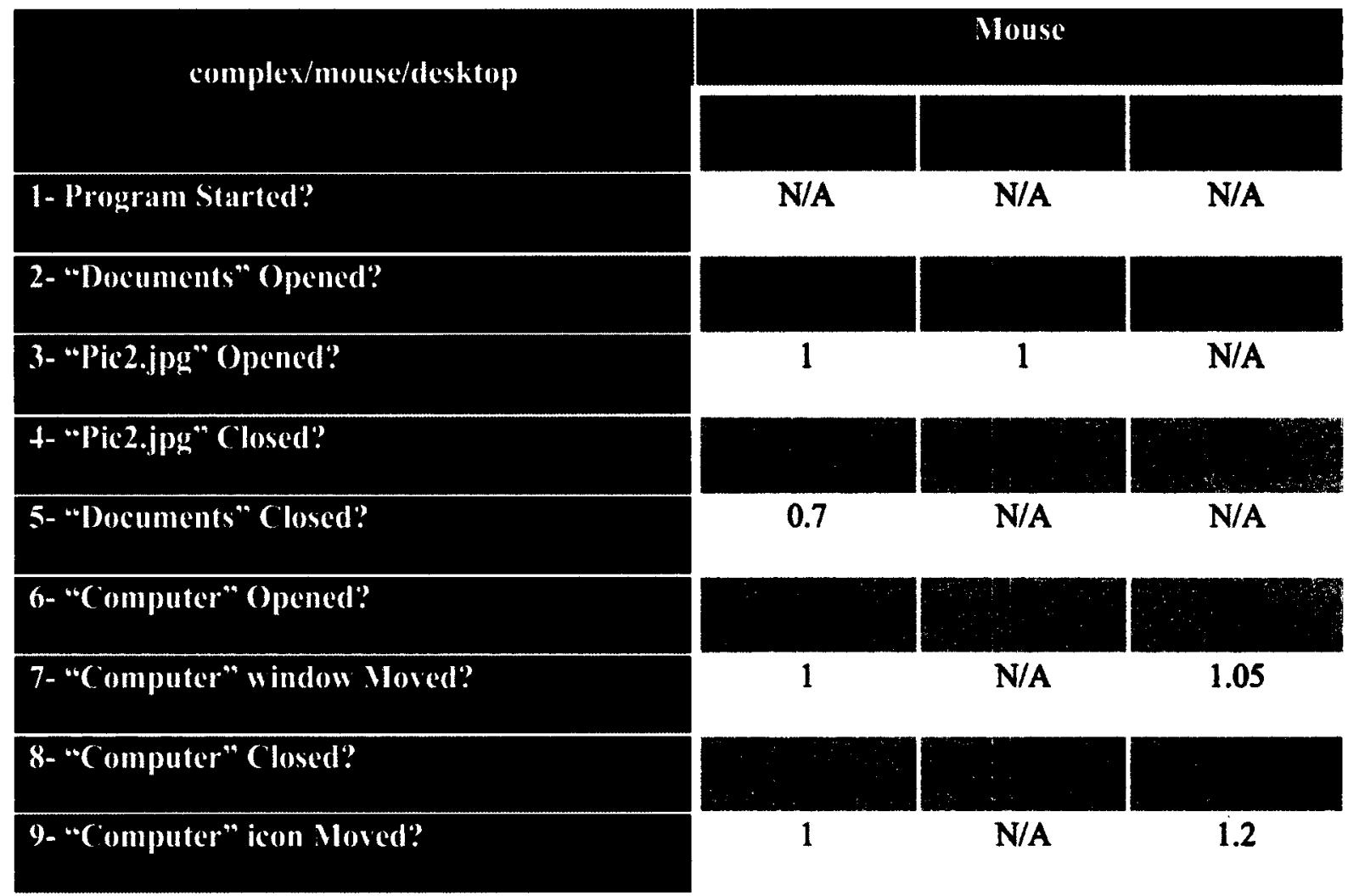

Table 5.15. Observation for complex task using mouse on big-screen.

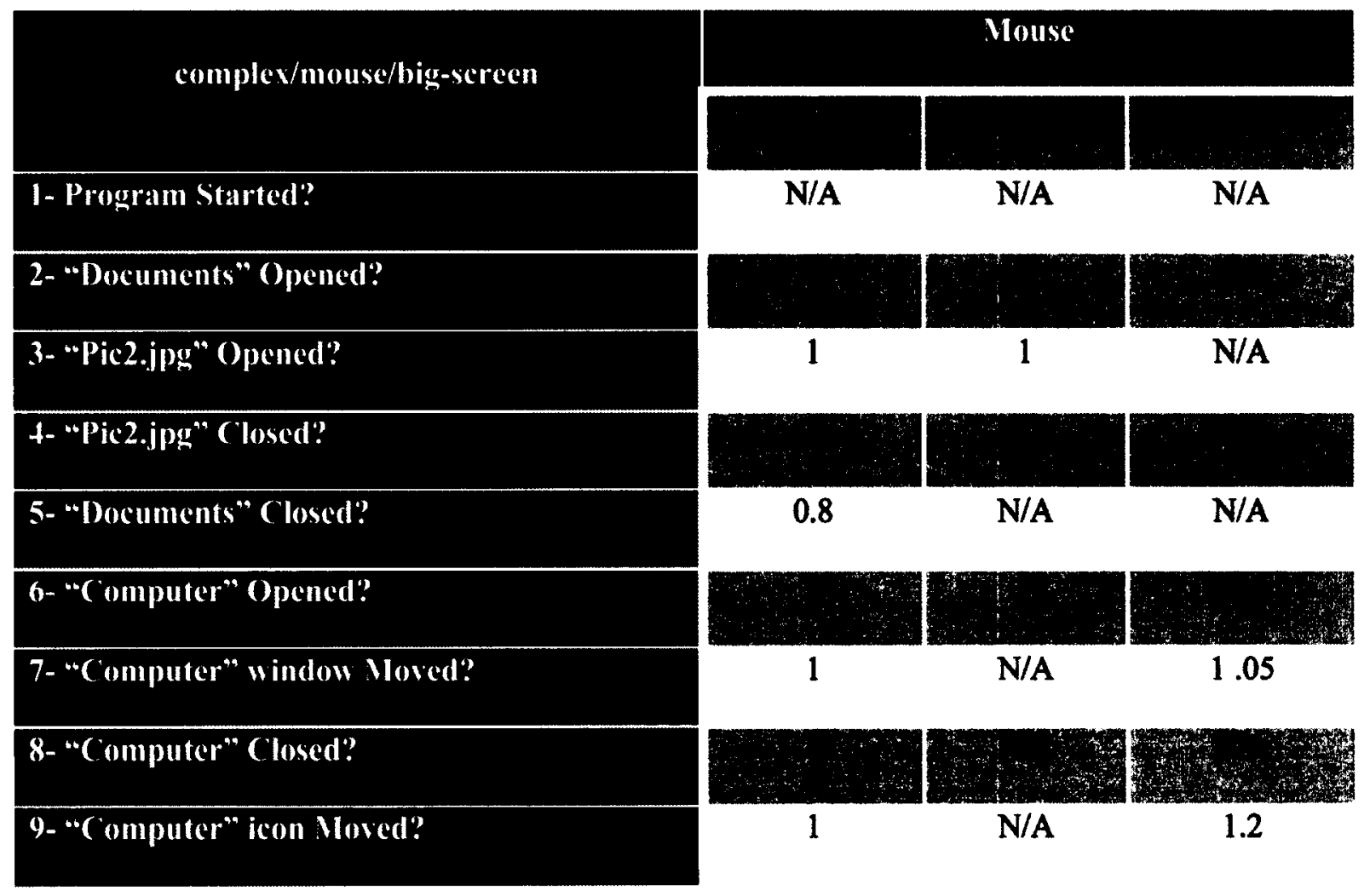


Table 5.16. Observation for complex task using gesture on desktop.

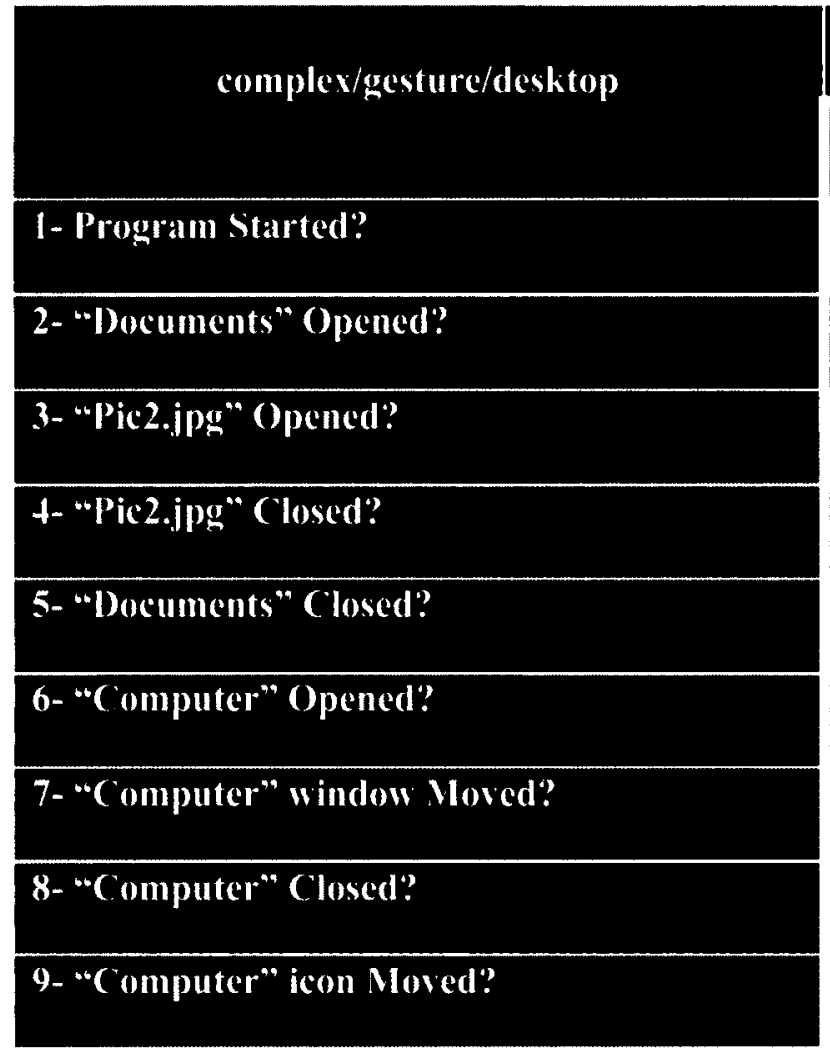

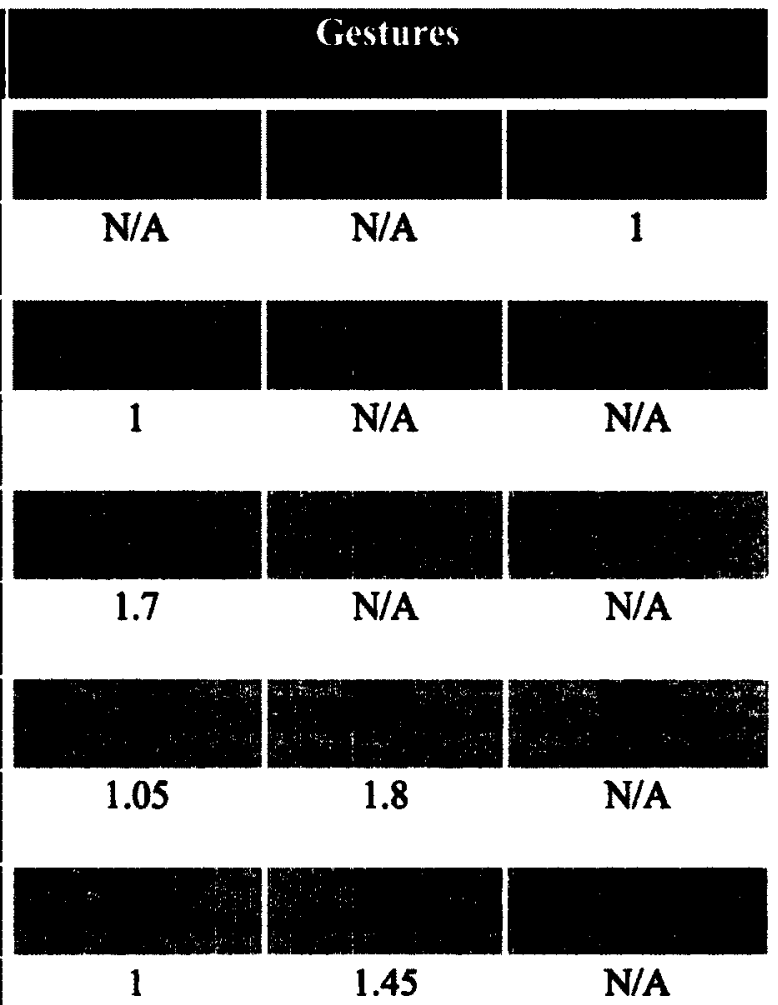

Table 5.17. Observation for complex task using gesture on big-screen.

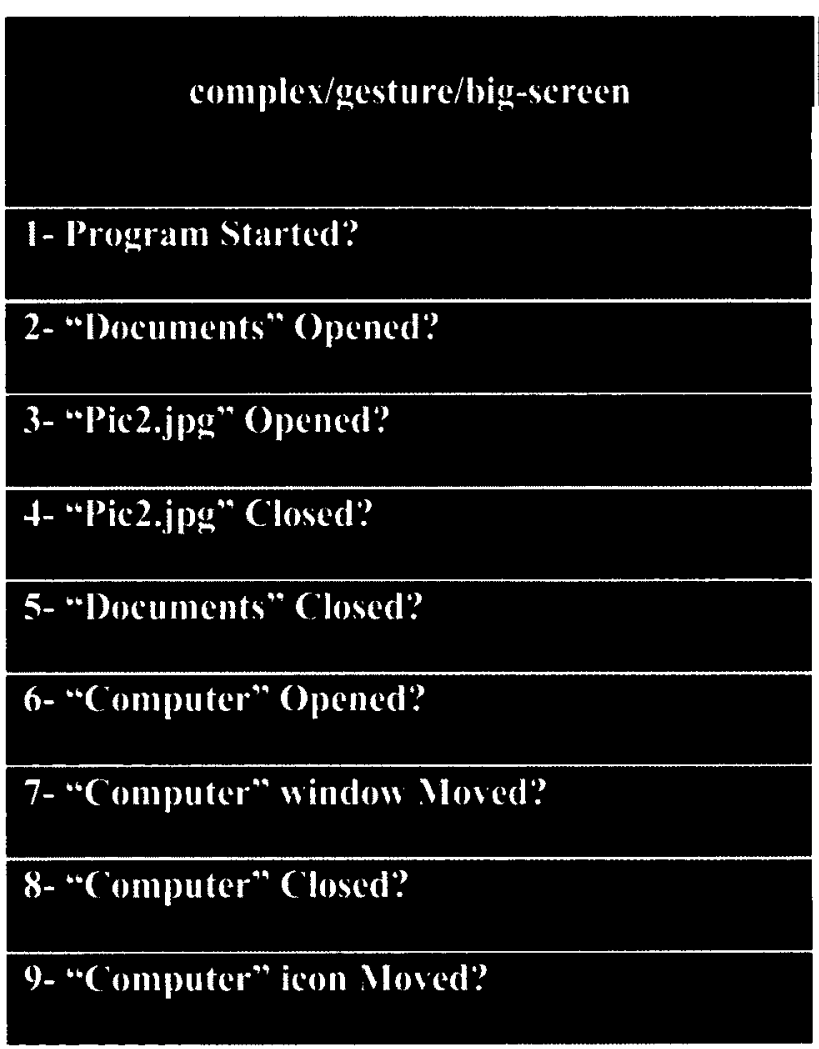

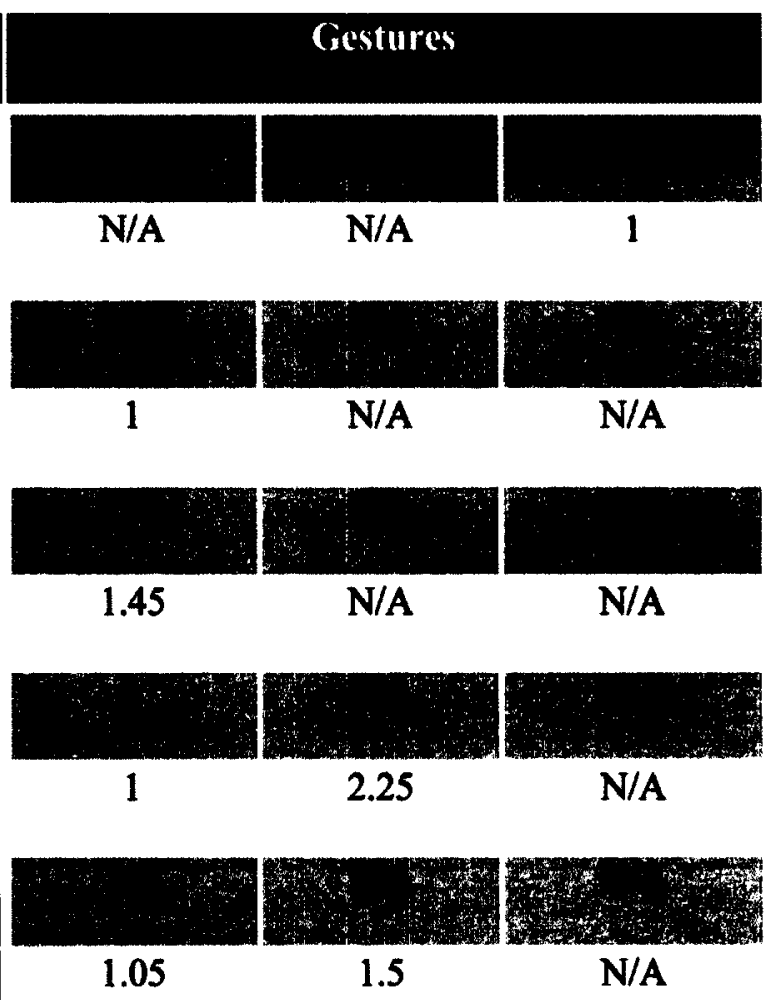




\subsubsection{Discussion}

\subsubsection{Hypotheses Verification}

According to the provided statistical analyses, we summarize our hypotheses verification as follows:

The time and the fatigue factors analyses support our initial hypotheses, meaning gesture inputs are significantly slower and more fatiguing than using a mouse. The initial hypotheses for the easiness and overall satisfaction factors are rejected which indicate that using a mouse is significantly easier than using arm gestures whiles neither inputs hold a significant popularity over the other. For the naturalness and the pleasure factors, the hypotheses are rejected as well, meaning arm gestures as inputs do not feel significantly more natural or more fun to use compared to mouse. However, it is revealed that using arm gestures on big-screen is significantly more natural and more pleasant than using a mouse on both the desktop and the big-screen. Also it is shown that arm gestures used on big-screen is significantly more pleasant compared to when it is used on desktop.

\subsubsection{Extra Observations}

\section{Timing:}

Using mouse on big-screen is slower than on desktop. As expected, due to not being familiar with controlling a UI using gestures, the result with mouse is faster than with gestures. However, we believe that having more practice and getting used to the gesture application, allows the users to perform the tasks almost as fast as using a mouse. 


\section{Satisfaction:}

Most of the participants preferred "equally use of mouse and gesture" as a combination of gesture and mouse inputs. User satisfaction data can be found in Appendix F.

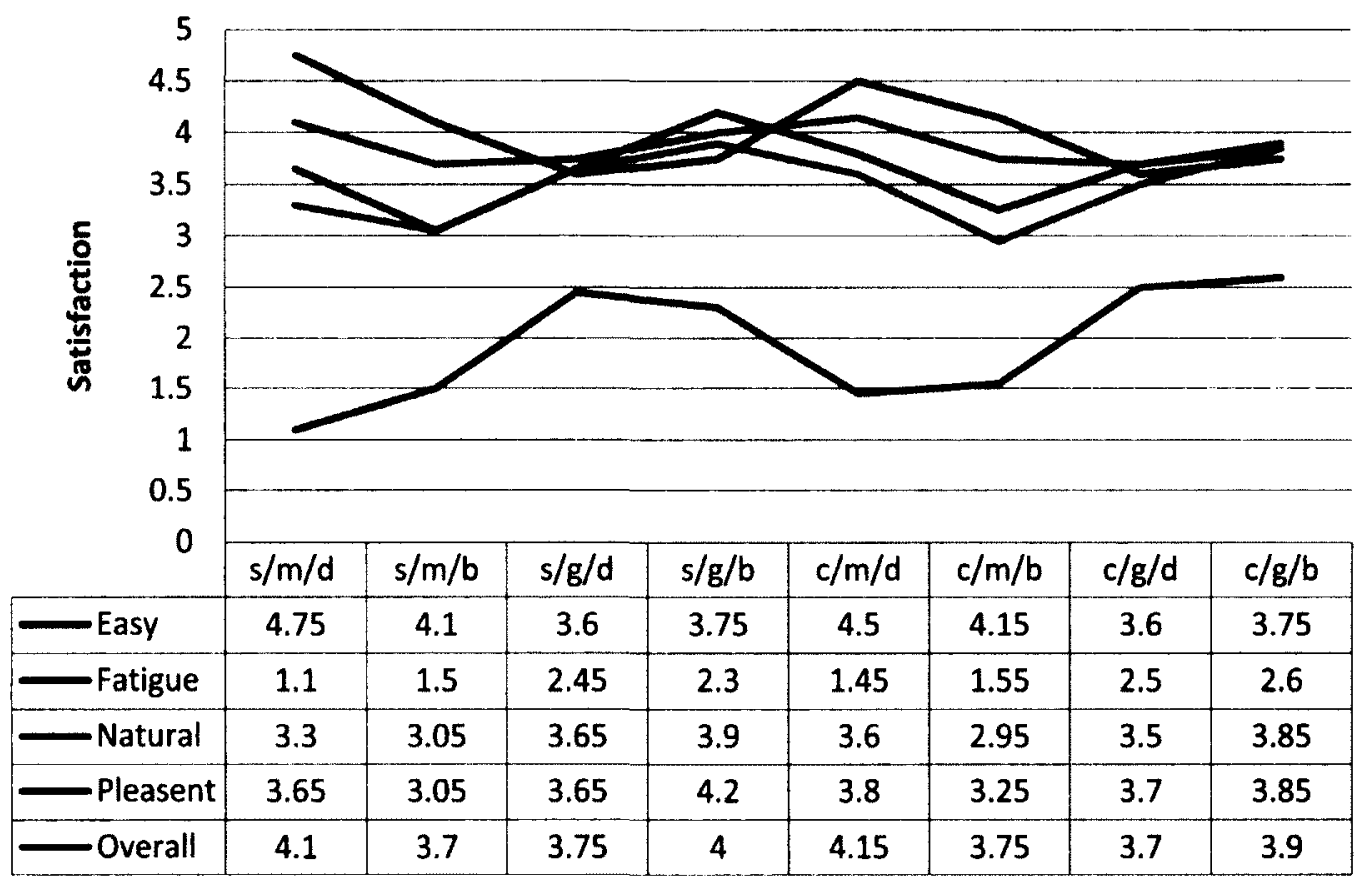

Figure 5.7. Satisfaction comparison ( $s=$ simple, $c \equiv$ complex, $m \equiv$ mouse, $g \equiv$ gesture, d=desktop, $b=$ big-screen). 


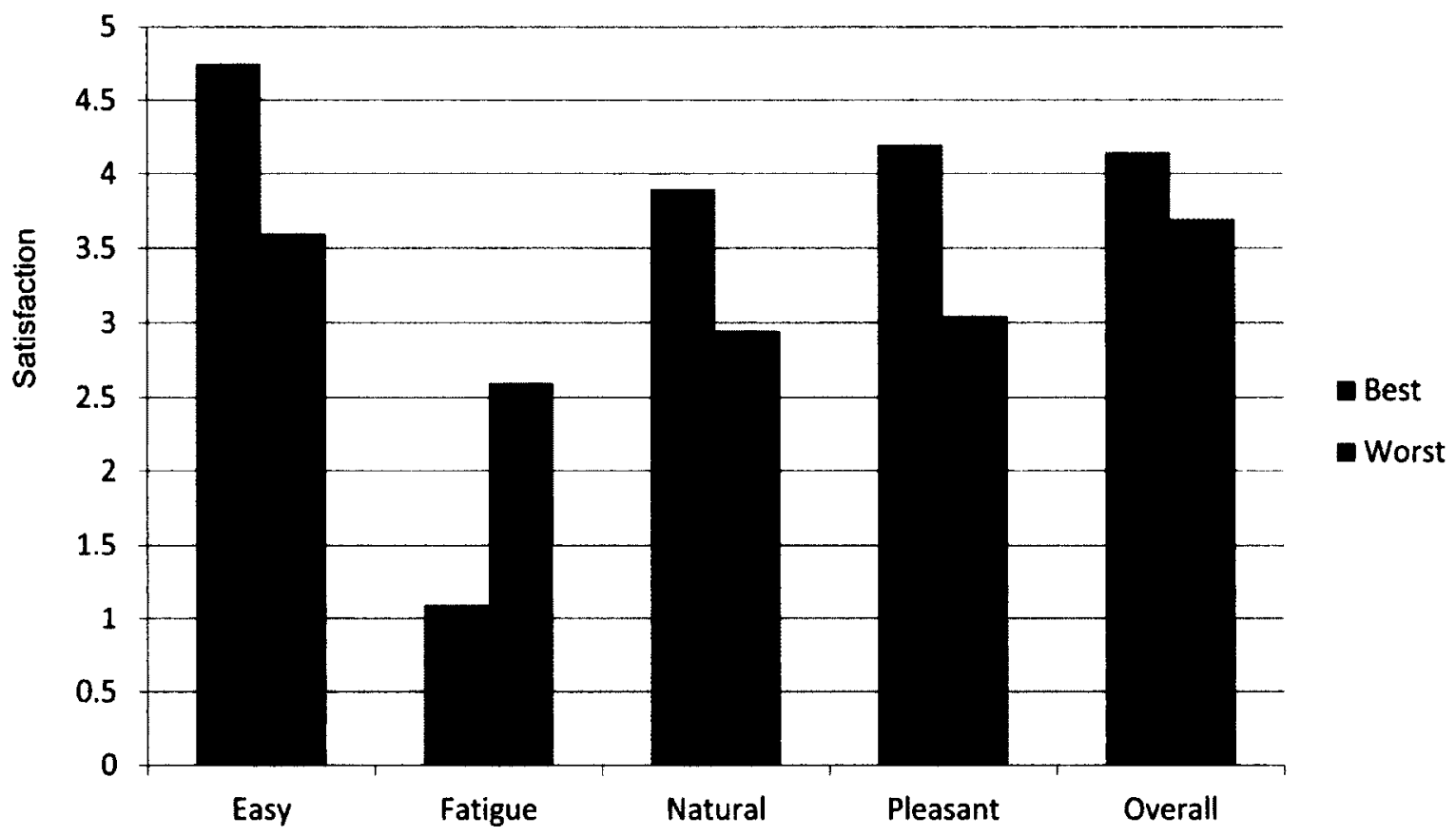

Figure 5.8. Best/Worst satisfactions $(s=$ simple, $c \equiv$ complex, $m \equiv$ mouse, $g=g e s t u r e$, $d=$ desktop, $b=b i g-s c r e e n)$.

As shown in Figure 5.8, doing simple-task with gestures on desktop caused more fatigue than on big-screen, although it is reverse in doing complex-task. Performing simple-task, using mouse on desktop is the easiest and the lightest (least fatigue) and on big-screen is the least pleasant and the least overall satisfactory, while using gestures on big-screen is the most natural, and the most pleasant. In addition, the complex-task using gestures on desktop is the most difficult and the least overall satisfactory. In other words, a short time usage of mouse on big-screen, and a long term usage of gesture on desktop have the least popularity from users' feedback. Doing complex-task, using mouse on desktop is the most overall satisfactory and on big-screen is the least natural, while using gesture on big-screen is the heaviest (most fatigue). 
As shown in Figure 5.9, opening a window (Running action) using gesture was the easiest task overall.

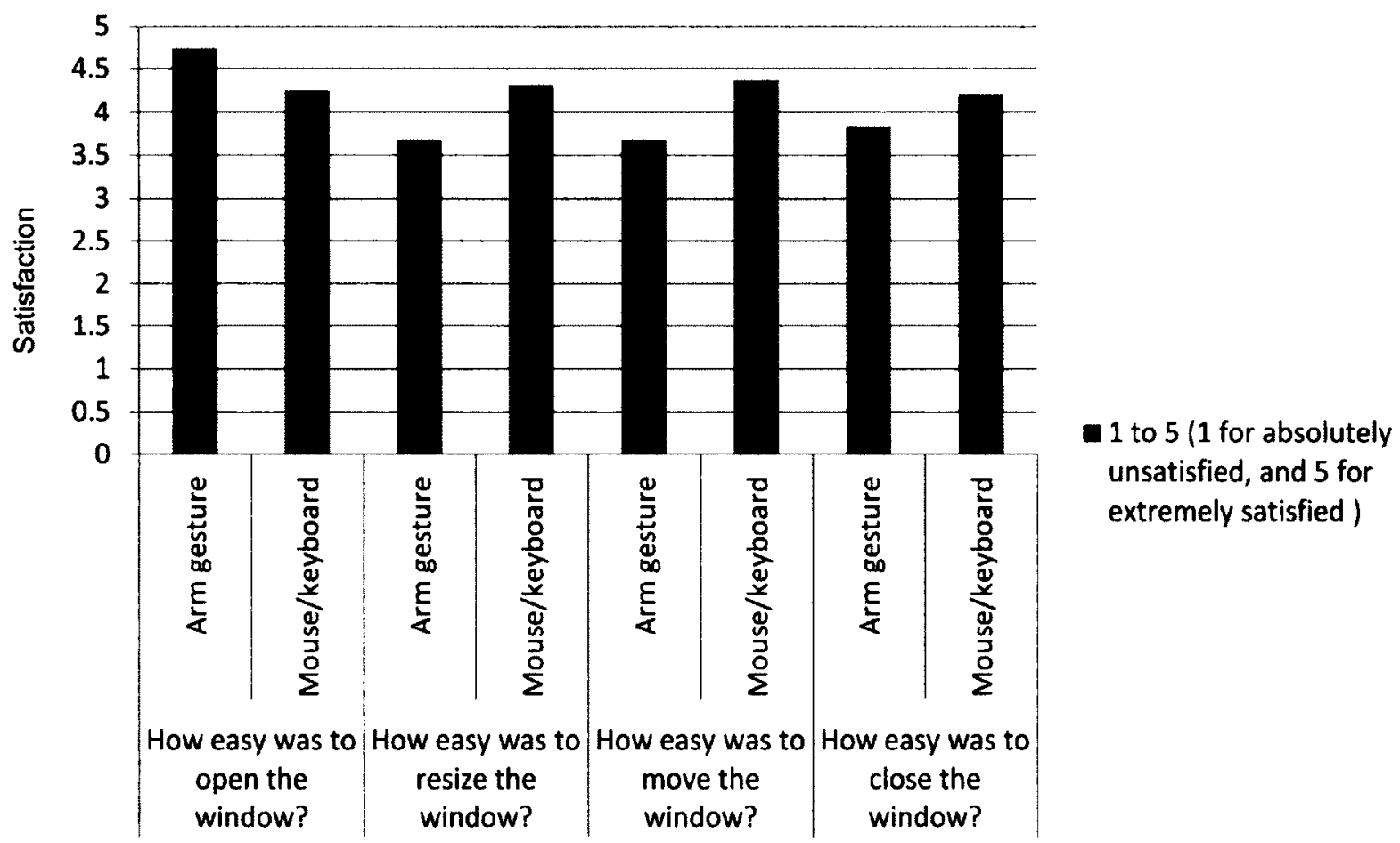

Figure 5.9. Four primitive tasks.

This study compared arm gestures with mouse/keyboard in two different settings (desktop and large-scale displays), and two different task difficulties (simple and complex). Combined UI makes more attentive and immersive than the conventional UI. There are still remaining issues to solve such that users feel fatigue while using arms in the air, which will enable the gestural interaction to be commonly used. 


\subsection{Phase 2: Finger Gestures vs. Arm Gestures}

\subsubsection{Study details}

This study is conducted using 10 participants. We performed this experiment just on bigscreen. However, it is on our future plan to expand the experiment by having more participants (at least 20), and testing on desktop as well as big-screen. Participants include students from Carleton University, and professionals from the OCE and Ottawa hospital. Student participants were Masters and $\mathrm{PhD}$ students from different departments including Information Technology, Computer Science, and Systems and Computer Engineering. They ranged in age from 26 to 36 years. The average age of participants was 30 years old. Some participants were familiar with the use of arm gestures (from the experiment in phase 1), but have not experienced finger gestures before. The participants first read the experiment instructions and were given introductions to the task they were to complete during the trial. They completed the trial at Interactive Media Group lab (iMG) at Carleton University. First, participants were introduced to the steps they had to follow to complete the tasks. A stopwatch registered the time taken to do each task. Each participant completed an individual 30 minutes trial.

The trial was divided into two phases:

- Training phase

- Test phase and satisfaction phase to complete a paper questionnaire 


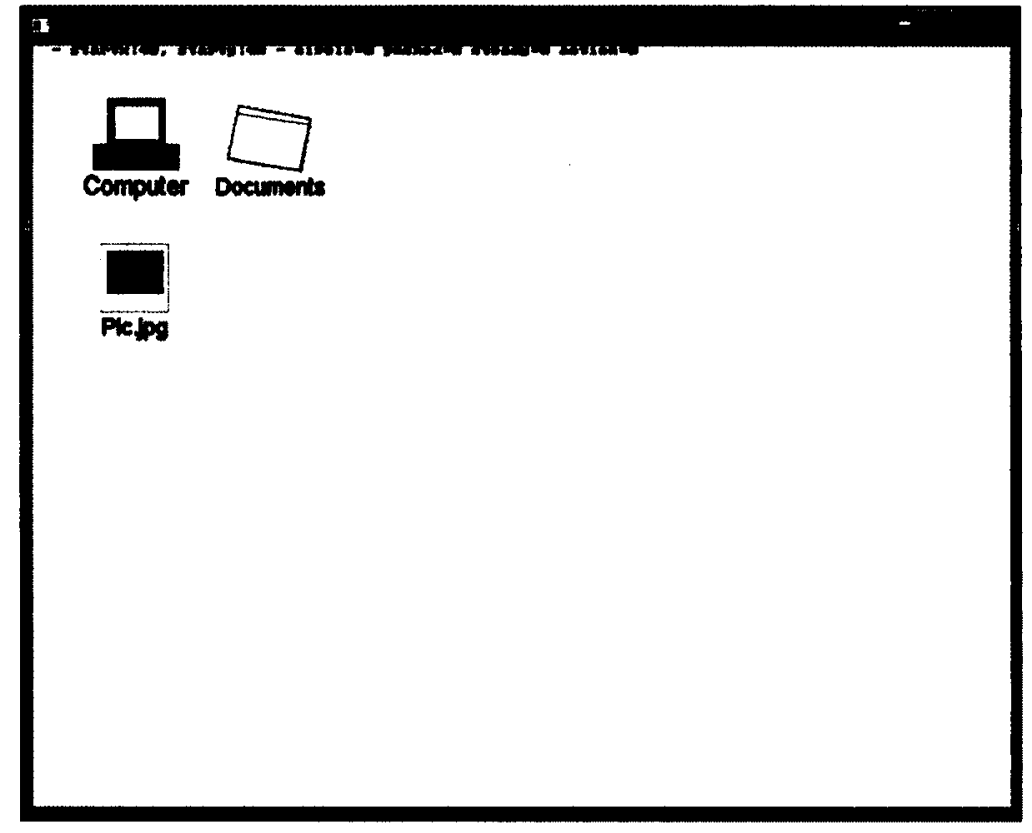

Figure 5.10. Interface.

In the satisfaction phase, participants were asked to complete a paper questionnaire. The aim of the questionnaire was to get the opinions of the group on using the two test methods and what they perceived as difficulties while completing the task.

\subsubsection{Results and Evaluation}

\subsubsection{Hypotheses and Statistical Analyses}

For the different factors being studied, 2-way repeated analysis of variances (ANOVA) is carried out for two independent variables:

1- Difficulty (simple task vs. complex task)

2- Input device (finger gestures vs. arm gestures)

All experiments were carried out on the big-screen and at $p<0.05$ significance level and for 10 participants. 


\section{Time:}

One researcher was taking the time with a stopwatch and one researcher counting the errors.

Table 5.18. Task duration.

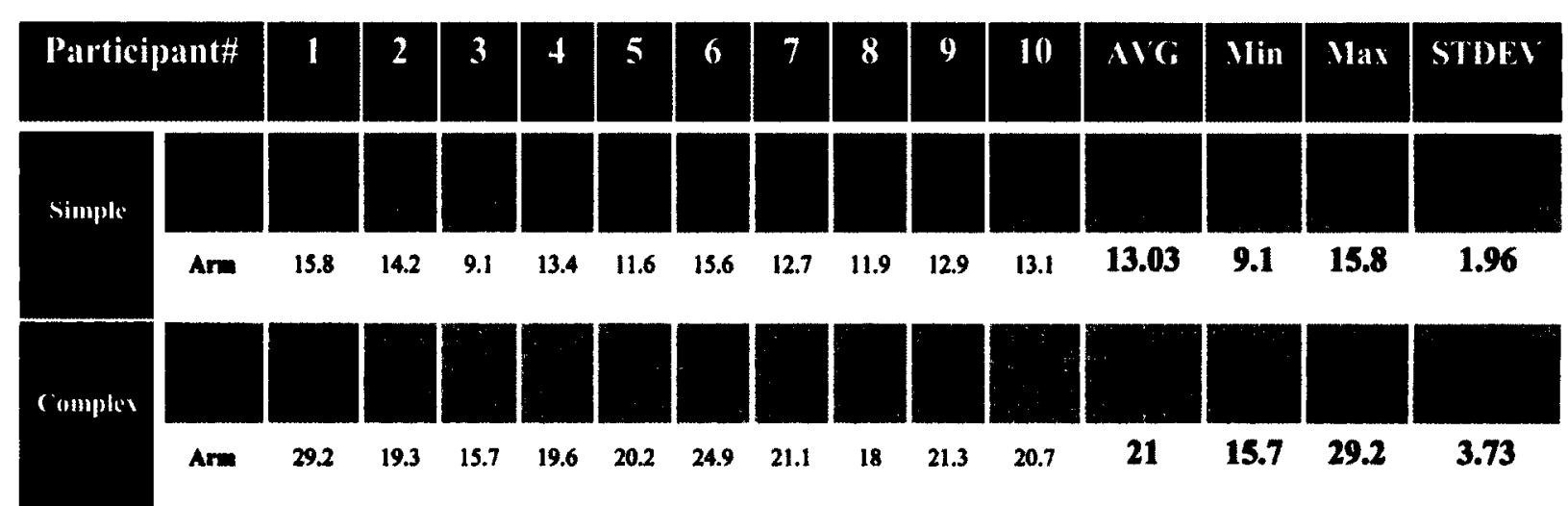

Table 5.18 shows the times taken to complete simple task and complex task once using arm gestures and once the finger gestures.

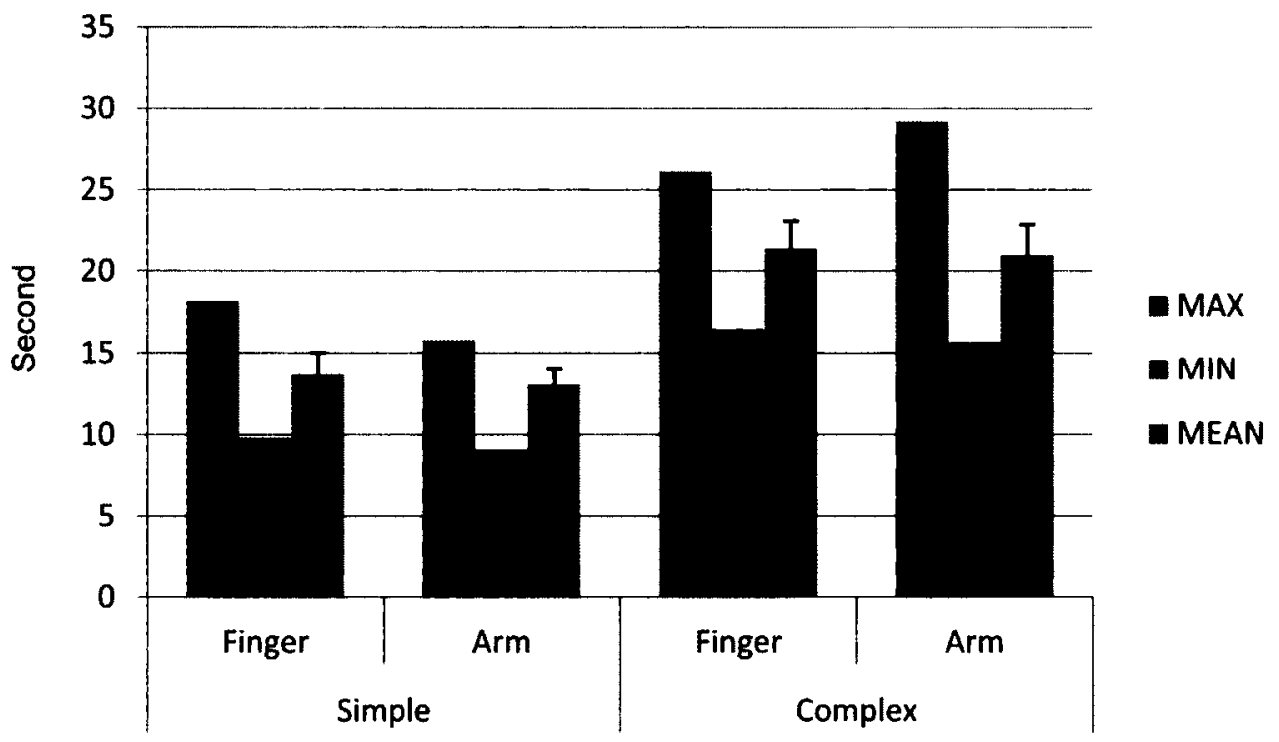

Figure 5.11. Temporal MAX/MIN/MEAN/ST DEV facts. 
Hypothesis- Using finger gestures is faster than using arm gestures as inputs.

The analysis shows $F(1,617.0102)=202.5032, P=0.0000$ for variable 1 , meaning task complexity has significant effect on time $\left(\mathrm{M}_{\text {simple }}=13.3550, \mathrm{SD}_{\text {simple }}=2.3027 \mathrm{vs}\right.$. $\mathrm{M}_{\text {complex }}=21.2100, \mathrm{SD}_{\text {complex }}=3.4385$ ). Similar to the first set of experiments, this trend is anticipated since the experiments were designed to maintain different complexities, thus durations. Variable 2 shows no significant effect on time, $F(1,2.8622)=0.3104, P=$ 0.5910 . Combination of variable also show no significant effect, $F(1,0.1323)=0.0503, P$ $=0.8275$, meaning there is no interaction between variables 1 and 2 . Based on the above, the hypothesis is rejected, implying that in terms of time, finger and arm maintain similar performances.

\section{Easiness:}

Hypothesis- Using finger gestures is easier than using arm gestures as inputs.

When analyzing participant feedback, for easiness, variable 1 shows $F(1,0)=0, P=1$ and variable 2 shows, $F(1,0)=0, P=1$. This means according to participants, neither variable has significant effect on easiness. For variables 1 and 2 combined, $F(1,0.1000)=2.2500$, $P=0.1679$ which indicates there is no effect for combination of variables and for easiness, there is no interaction between the two. According to the analysis, the hypothesis is rejected meaning neither finger gestures nor arm gestures are significantly easier than the other.

\section{Fatigue:}

Hypothesis- Using finger gestures causes less fatigue than using arm gestures as inputs. 
In this experiment the participants have been asked to rank higher if less fatigue is experienced. The results for variable 1 shows no significant effect $F(1,0)=0, P=1$. Variable 2, the input, shows significant effect $\mathrm{F}(1,4.9000)=12.2500, \mathrm{P}=0.0067\left(\mathrm{M}_{\text {finger }}\right.$ $=4.5000, \mathrm{SD}_{\text {finger }}=0.6070$ vs. $\left.\mathrm{M}_{\mathrm{arm}}=3.8000, \mathrm{SD}_{\mathrm{arm}}=0.6959\right)$. This indicates that using fingers rather than arm significantly reduces the fatigue caused to participants. Finally variables 1 and 2 combined show no significant effect $F(1,0)=0, P=1$. According to the figures mentioned above, the hypothesis is confirmed implying that the fatigue caused by arm is significantly higher than that of fingers.

\section{Naturalness:}

Hypothesis- Using finger gestures is more natural than using arm gestures as inputs.

For this parameter, variable 1 shows $\mathrm{F}(1,0.0250)=1.0000, \mathrm{P}=0.3434$, indicating no significant effect. Variable 2 results in $F(1,11.0250)=441.0000, P=0.0000\left(M_{\text {finger }}=5\right.$, $\mathrm{SD}_{\text {finger }}=0$ vs. $\mathrm{M}_{\mathrm{arm}}=3.9500, \mathrm{SD}_{\text {arm }}=0.2236$ ) indicating that use of fingers feels extremely more natural to participants. For interaction of the two variables shows no significant effect with $F(1,0.0250)=1.0000, P=0.3434$. According to the analysis, the hypothesis that finger gestures are more natural as inputs compared to arm gestures is confirmed.

\section{Pleasantness:}

Hypothesis- Using finger gestures is more pleasant than using arm gestures as inputs. Analyzing the feedback for pleasantness according to participants, it is revealed that neither variables have an advantage over the other in this regard. For variables 1, 
$\mathrm{F}(1,0.4000)=6.0000, \mathrm{P}=0.0368\left(\mathrm{M}_{\text {simple }}=4.8000, \mathrm{SD}_{\text {simple }}=0.4104\right.$, vs. $\mathrm{M}_{\text {complex }}=$ $\left.4.6000, \mathrm{SD}_{\text {complex }}=0.5026\right)$ indicating that simplicity has significant effect. For variable 2, $F(1,0)=0, P=1$, meaning there is no significant effect. For variables 1 and 2 , $F(1,0.4000)=6.0000, P=0.0368$, indicating there is significant interaction between the two. Multiple one-way ANOVAs further indicate that finger gestures are significantly more pleasant for simple tasks rather than complex ones $\left(\mathrm{M}_{\text {finger-simple }}=4.9000, \mathrm{SD}_{\text {finger- }}\right.$ simple $=0.3162$ vs. $\mathrm{M}_{\text {finger-complex }}=4.5000, \mathrm{SD}_{\text {finger-complex }}=0.5270$ ). Therefore, the hypothesis that finger gestures compared to arm gestures are more pleasant to employ as inputs is rejected.

\section{Overall Satisfaction:}

Hypothesis- Overall, using finger gestures as inputs is a more popular experience compared to arm gestures.

The overall experience by participants shows no significant effects for variable 1 , $\mathrm{F}(1,0.4000)=3.2727, \mathrm{P}=0.1039$ and variable $2, \mathrm{~F}(1,0.4000)=3.2727, \mathrm{P}=0.1039$. Moreover, there is no interaction between variables 1 and $2, F(1,0.1000)=2.2500, P=$ 0.1679 . Accordingly, the hypothesis is rejected, meaning that both experiences maintain similar popularities among participants.

\subsubsection{Number of Errors}

The following tables show the average number of trials before all participants successfully perform a task (average number of errors in each gestural task for all 10 users). 
Table 5.19. Observation for simple task using finger on big-screen.

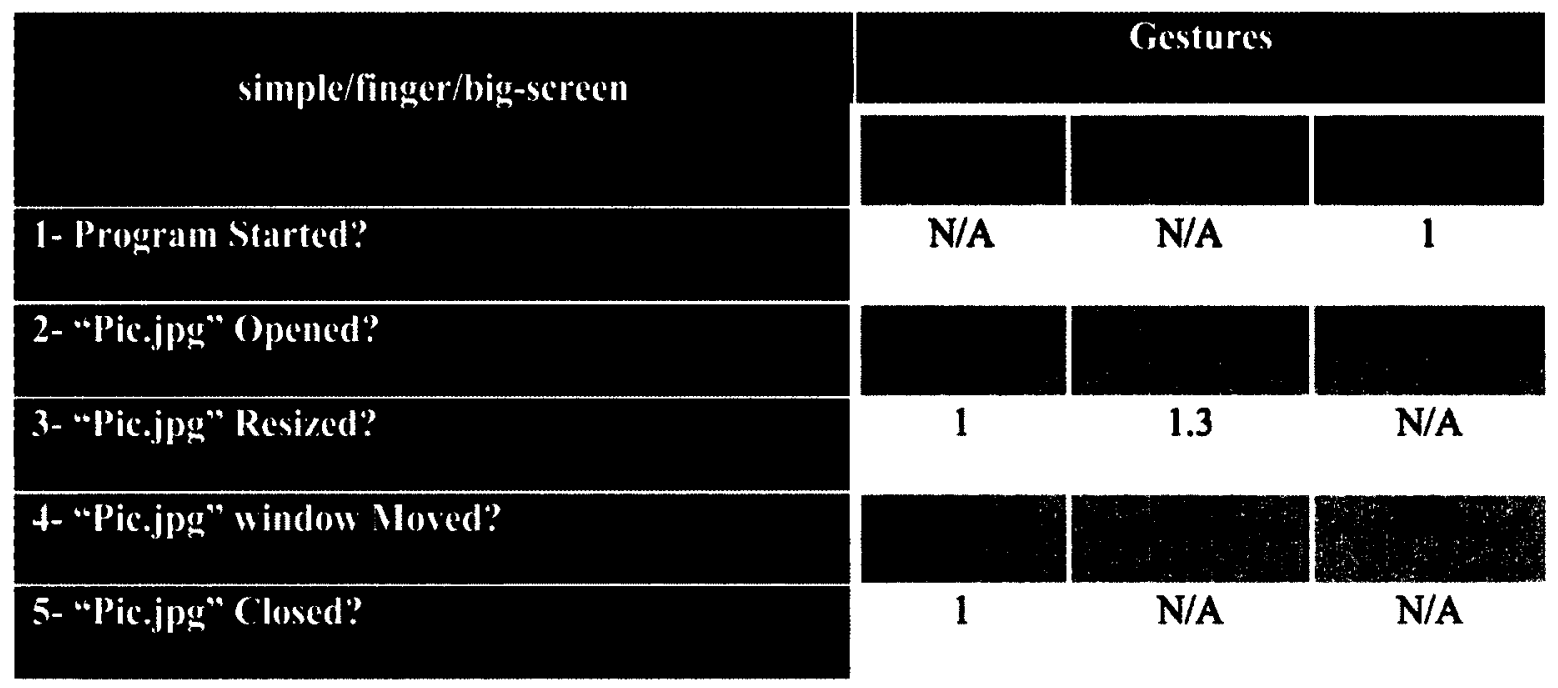

Table 5.20. Observation for simple task using arm on big-screen.

\begin{tabular}{|l|}
\hline \multicolumn{1}{|c|}{ simple/arm/lig-screen } \\
\hline 1- Program Started"? \\
\hline 2- "Pic.jpg" Opened?' \\
\hline 3- "Pic.jpg" Resized? \\
\hline 4- "Pic.jpg" window .loved? \\
\hline 5- "Pic.jpg" Closed? \\
\hline
\end{tabular}

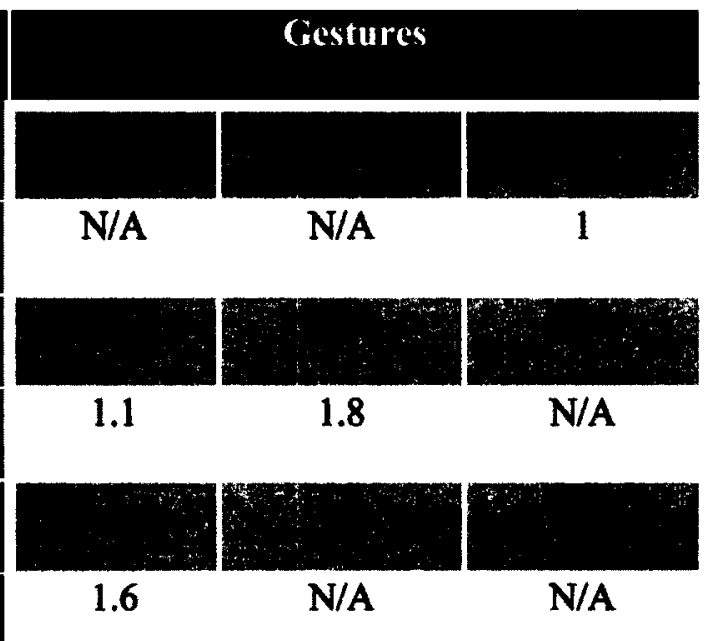


Table 5.21. Observation for complex task using finger on big-screen.

\begin{tabular}{|l} 
complex/finger/big-screen \\
\hline 1- Program Started"? \\
\hline 2- "Documents" Openced" \\
\hline 3- "Pic2.jpg" Opened" \\
\hline 4- "Pic2.jpg" Closed"? \\
\hline 5- "Documents" Closed"? \\
\hline 6- "Computer" Opened"' \\
\hline 7- "Computer" window Moved"? \\
\hline 8- "Computer" Closed"? \\
\hline
\end{tabular}

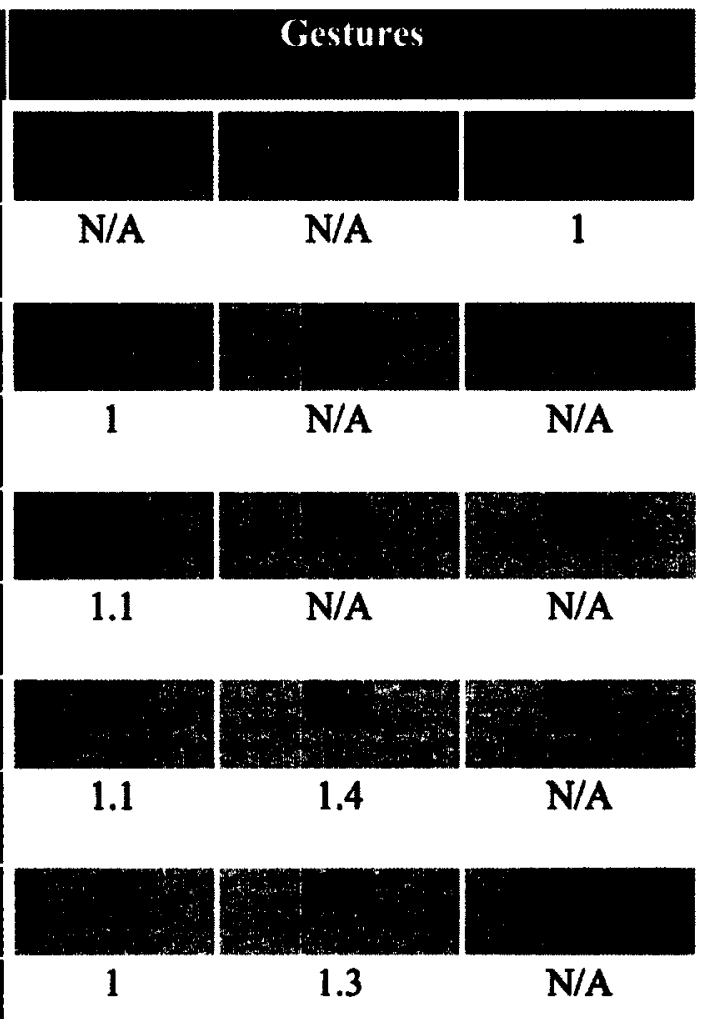

Table 5.22. Observation for complex task using arm on big-screen.

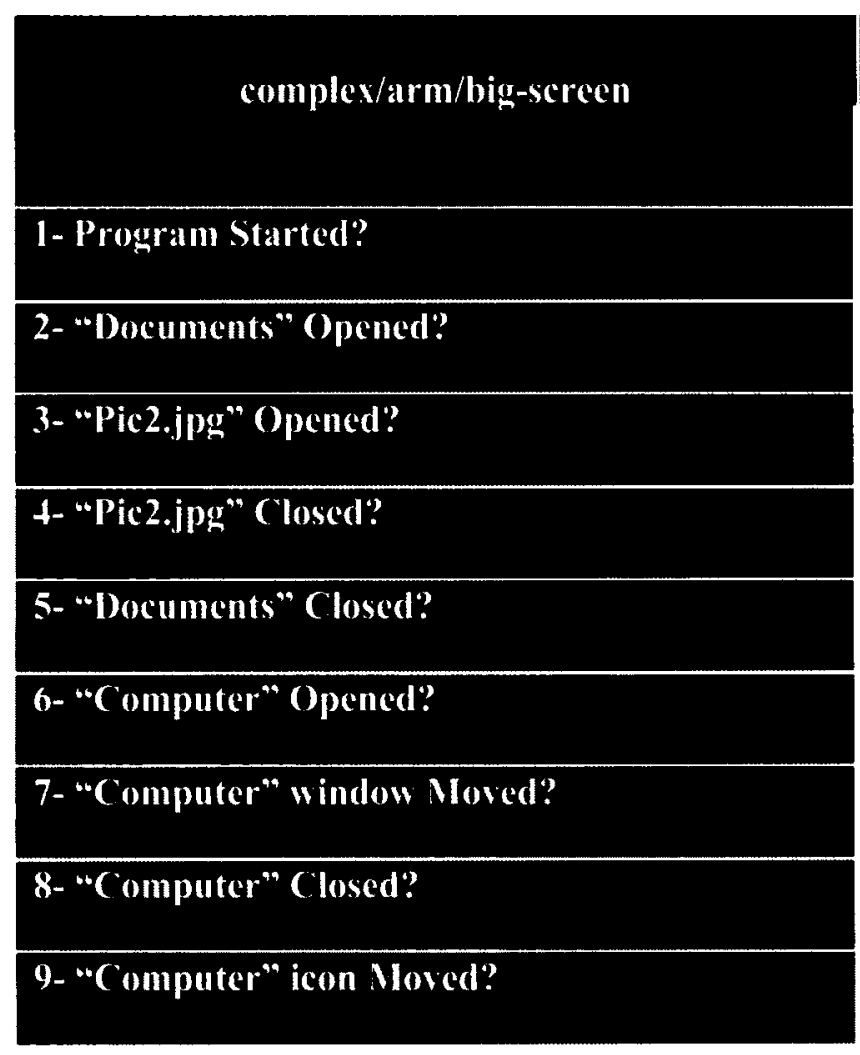

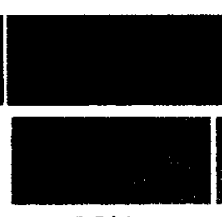

N/A

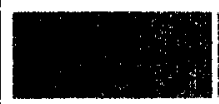

1

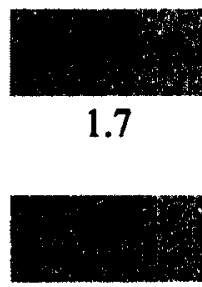

1.1

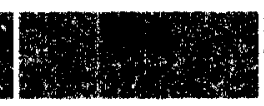

1.2

N/A

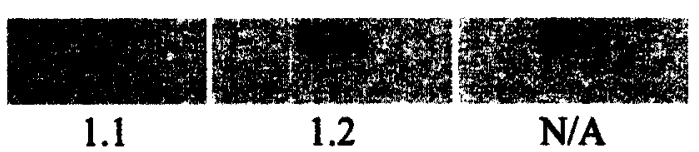




\subsubsection{Discussion}

\subsubsection{Hypotheses Verification}

According to the provided statistical analyses, we summarize our hypotheses verification as follows:

In general, the main result of this experiment (phase 2) is that fatigue is less with finger vs. arm gestures. To elaborate on, the naturalness and the fatigue factors analyses support our initial hypotheses, meaning the finger gestures significantly are more natural and cause less fatigue as inputs compared to the arm gestures.

The initial hypotheses in terms of time, overall satisfactory, and easiness are rejected, implying that finger and arm maintain similar performances and popularities among participants, and neither finger gestures nor arm gestures are significantly easier than the other. Moreover, for the pleasure factor, the initial hypothesis is rejected as well, meaning the finger gestures compared to the arm gestures are not significantly more pleasant to employ as inputs. However, it is revealed that finger gestures are significantly more pleasant for simple tasks rather than complex ones.

Anecdotal evidence of natural grabbing: Before disclosing the defined gestures to the participants, we have asked them to try grabbing an object (here an icon) naturally and based on their common sense. The result was surprising: $85 \%$ of them in their first guess could correctly pick an object on screen by Pinching gesture. This survey illustrates that how successfully we have defined our natural finger gestures. 


\subsubsection{Extra Observations}

\section{Satisfaction:}

Most of the participants preferred "mostly finger" as a combination of arm and finger gestures. User satisfaction data can be found in Appendix F.

As shown in Figure 5.13, using arm is easier in short time. However, it is easier to use finger in the long run. In addition, using finger in the short time (simple task) is the most pleasant, and in the long term (complex task) is the least pleasant. The overall satisfaction had its highest level on the simple task using finger gestures, and its lowest level on the complex task using arm gestures.

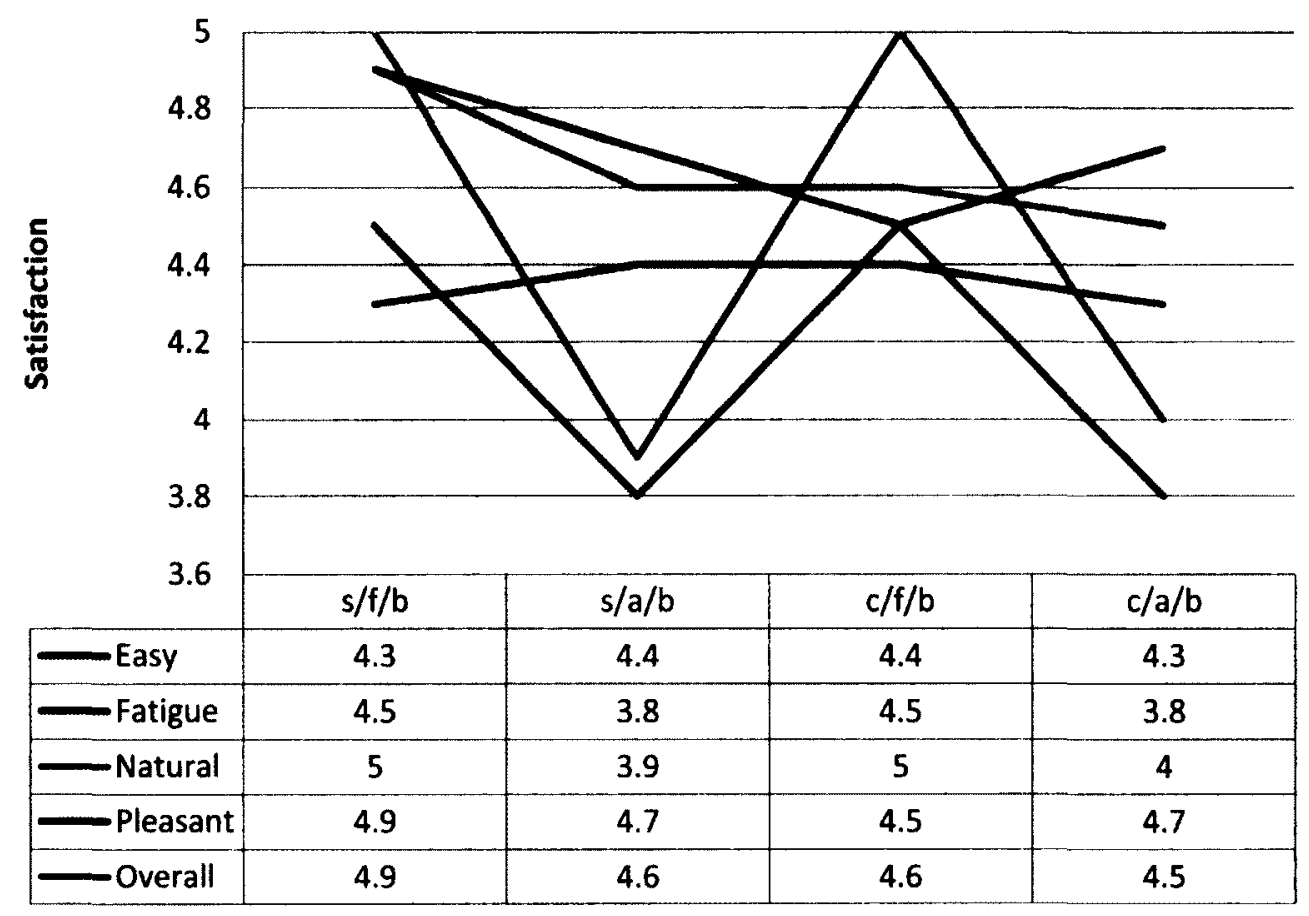

Figure 5.12. Satisfaction comparison $(s \equiv$ simple, $c \equiv c$ complex, $f=$ finger, $a=a r m, b=b i g-$ screen). 


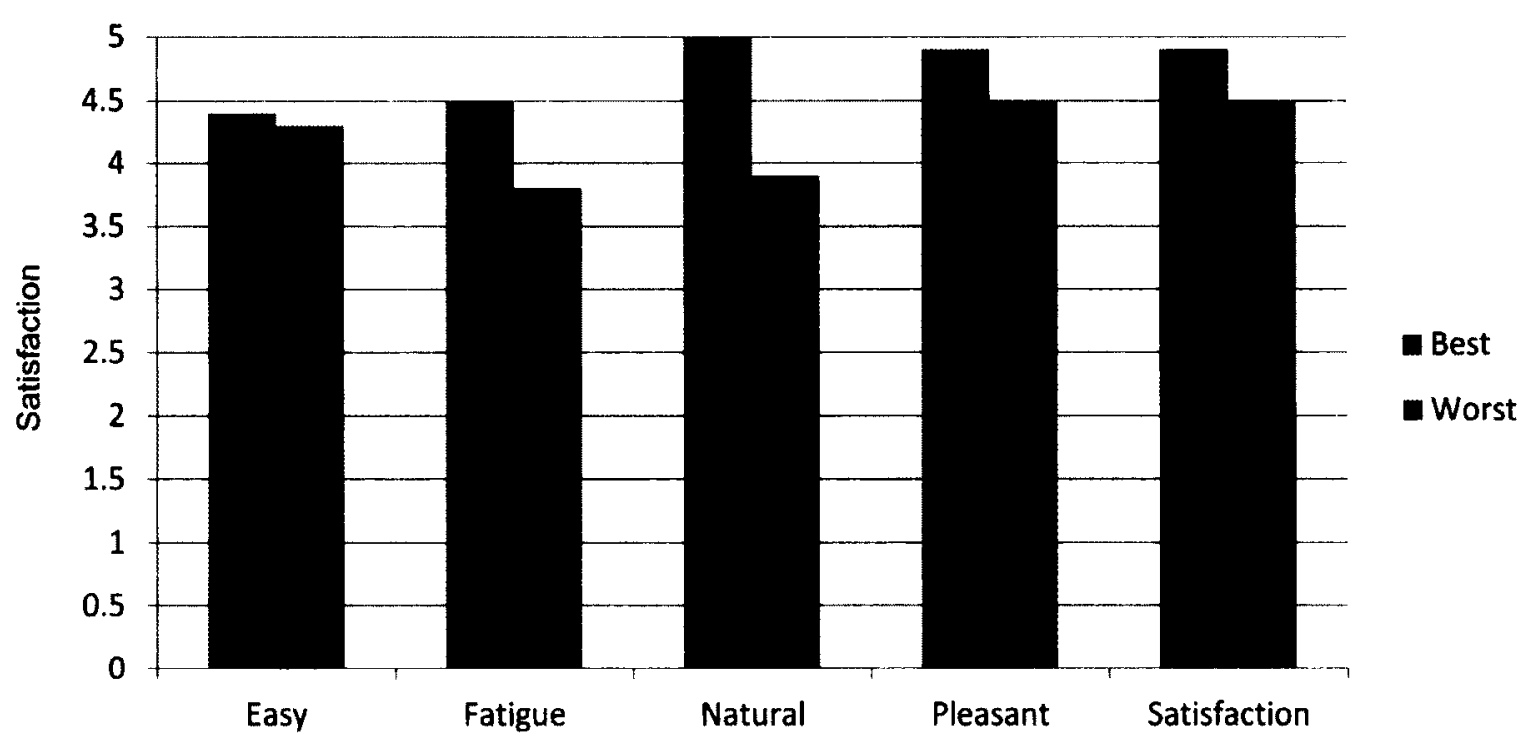

Figure 5.13. Best/Worst satisfactions ( $s=\operatorname{simple,~} c=c 0 m p l e x, f=f i n g e r, a=a r m, b \equiv b i g-$ screen).

As shown in Figure 5.14, the Pinching to resize and the Pushing to close a window were the most difficult gestures, due to the relatively small control access area of the objects.

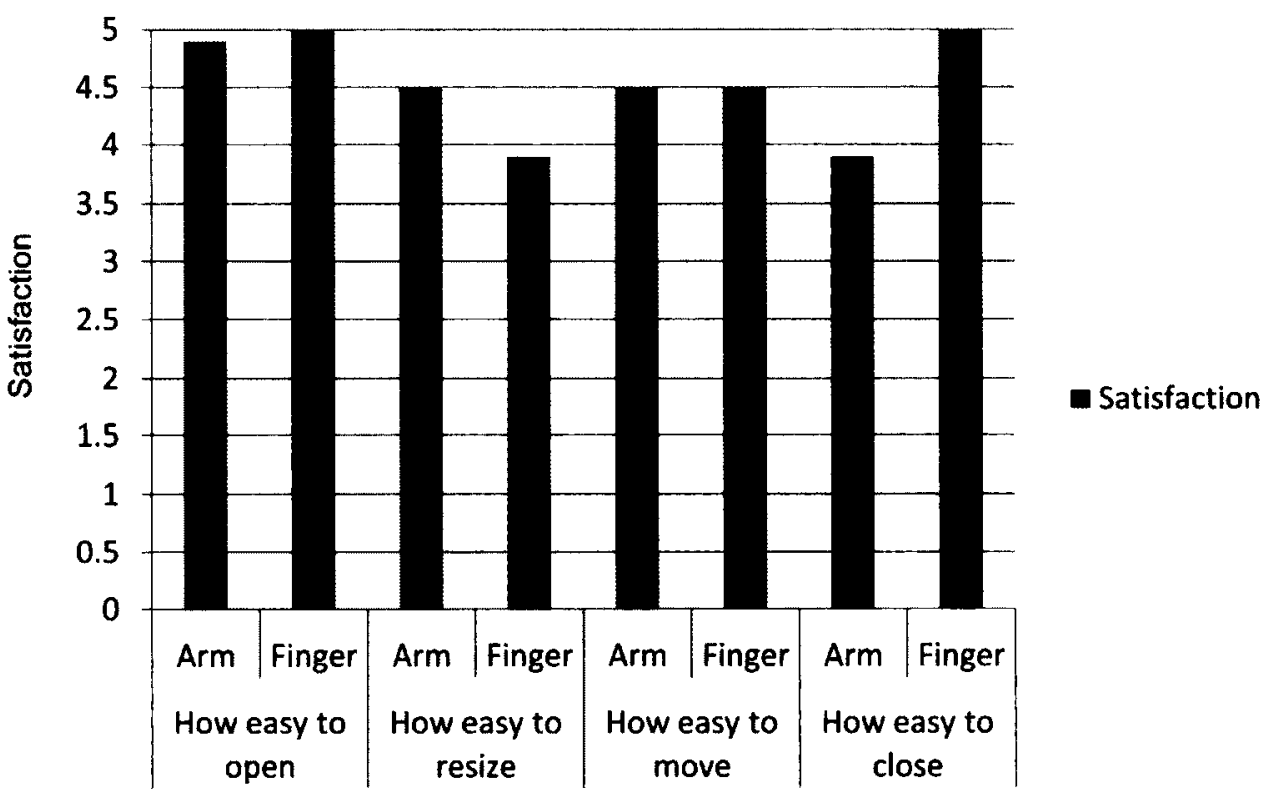

Figure 5.14. Four primitive tasks. 


\section{Errors:}

As the following figures show, the Tapping was the smoothest gesture with the least errors in both simple and complex tasks, while the Circling had the highest error in the simple task. However, the most errors happened with the Pushing during the complex task on the action of closing a window.

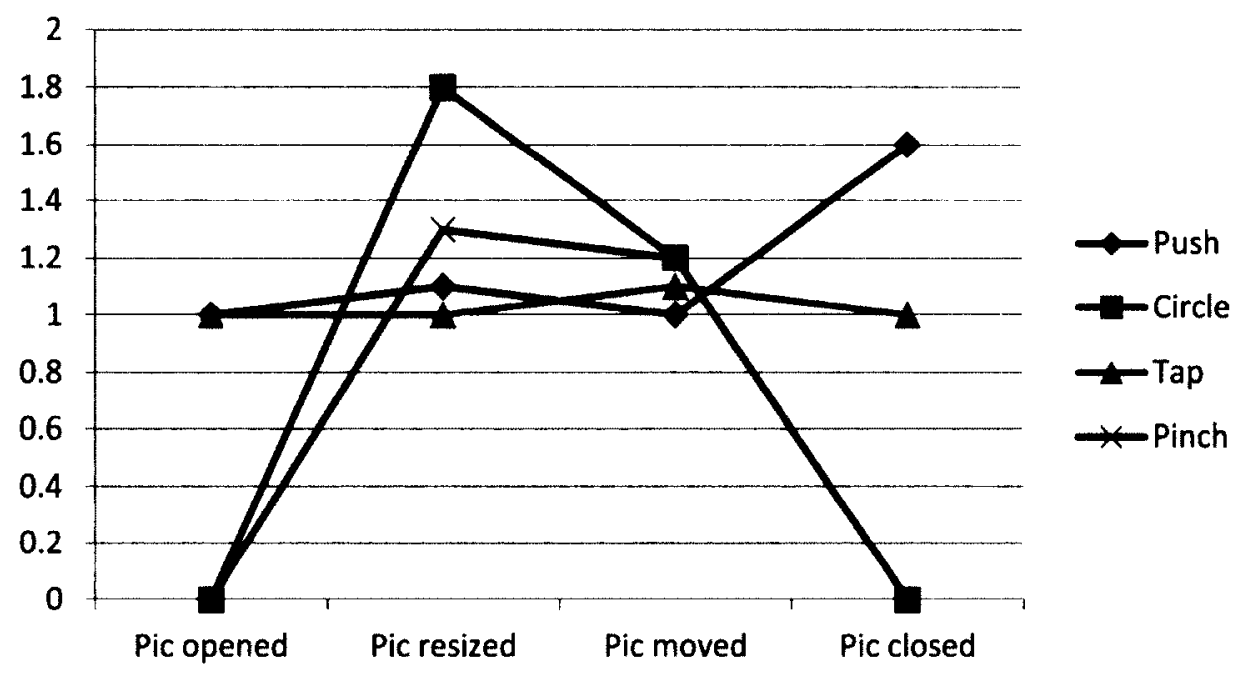

Figure 5.15. Gestures errors in simple task.

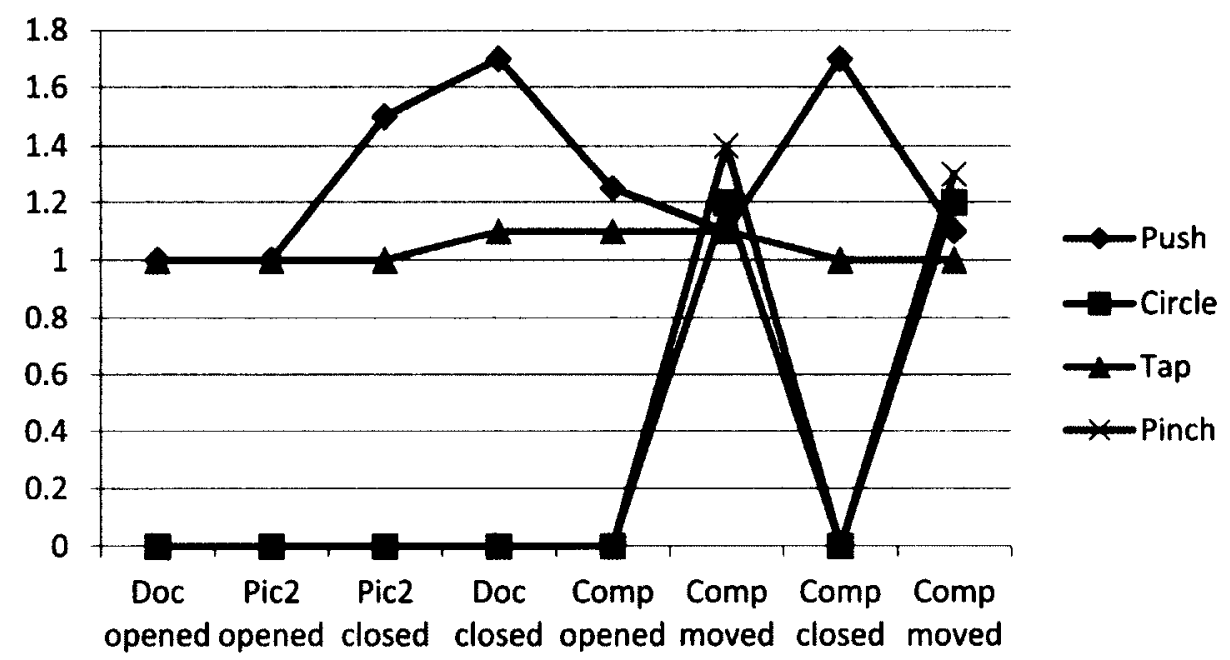

Figure 5.16. Gestures errors in complex task. 
This study compared finger-based gestures with arm-based gestures in two different settings of simple and complex tasks on a large-scale display. Combined UI makes more attentive and immersive than the UI solely controlled by one type of gestures.

\subsection{Sources of Error}

Although we have attempted to design and carry out this research as precise and robust as possible, the results of this study are still on the subject of improvement by decreasing the effect of errors. The sources of possible errors/inconsistencies involving in this project are as following:

- Users (lack of familiarity with gestures)

- User interface design

- Software/API's efficiency

- Equipment's capability

- Gestures definition/selection

- Gesture recognition algorithm

\subsection{Users' Comments Summary}

As it has been explained before, our questionnaire had a final part for the participants' comments (Appendix D) to share their opinions about the strengths and the weaknesses of using our gestural desktop application comparing to the conventional mouse-based application, after they have tested our prototype. Some of these comments, e.g. the effect of fatigue or naturalness factors, have been approved in our hypothetical analyses or been 
observed in our statistical graphs, e.g. the four primitive tasks satisfaction. This section summarizes their comments about the gestures as following:

- Freedom to move.

- Natural, convenient and attractive. By getting used to the environment it can be more convenient than a mouse.

- A good way to exercise during work.

- A more immersive experience.

- Probably better for your wrists (carpal tunnel, etc.).

- Arm gesture application is good for users with disabilities, e.g. not having fingers.

- Wireless mouse, having the same range of operation as gesturing, still requires flat surface to move the pointer. Not all flat surfaces comply with a laser or track ball mouse.

- Very helpful and feasible for some special purposes.

- Very interesting to be applied in seminars and big classrooms.

- It's more fun and exciting than just using a mouse. 


\section{Chapter 6: Conclusion}

The evolution of computer technologies have facilitated new ways of interaction between human and machine with a focus on robust and reliable approaches for more "natural" input methods, and particularly those through body motion tracking. The vision-based gesture recognition, the topic of this thesis, is one of these methods.

Although there has been considerable amount of research on this topic, there is a significant lack of standardization, inter-API compatibility, and especially usability analysis, for $\mathrm{HCI}$ methods using vision-based arm and hand gestures.

This thesis reviewed a series of alternative methods to replace the traditional way that human interact with a computer. The primary objective of this project was design and development of a prototype that enables users to use vision-based hand/arm gestures to perform common desktop operations like opening files, moving/resizing windows, etc. Our second objective was to perform a usability study in order to answer a series of 
questions and verify some hypotheses. This research has the following major contributions:

- A new gesture-based interface has been presented that provides a new way for controlling a simulated desktop by two sets of finger and arm gesture commands within a three dimensional environment. Employing the Kinect depth camera and OpenNI has secured our system with high stability and efficiency, while designing a capable algorithm using NITE and OpenCV has enabled our prototype to recognize the arm and finger gestures effectively.

- Our choice of gestures implemented in the system is based on the theory of bi-manual gestures activities [82]: the preferred gestures are used where precision/satisfaction is necessary. The tasks are performed more natural, easily, and in some points faster using the combination of the three sets of controllers: mouse, arm gestures, and finger gestures.

- Finally, through a comprehensive and hypothetical user experiment we compared our natural defined gestures (finger and arm) to each other and also to the conventional input devices (mouse/keyboard), in two different settings (desktop and big screen displays), and during two sets of tasks (simple and complex) for precision, efficiency, easiness, pleasantness, fatigue, naturalness, and overall satisfaction to verify the following hypothesis: the gesture-based input is superior to mouse/keyboard when using big screen; and the finger-based gesture input is superior to arm-based in the long term of use. Moreover, our experiment has analytically proved that using gestures on a big-screen display is more natural and pleasant than using a mouse/keyboard in a $\mathrm{HCI}$. On the other hand, arm gestures are more fatiguing than 
mouse, although using the finger gestures have improved the results by reducing the fatigue factor comparing to the arm gestures.

Multi-user, multi-modal, and multi-dimensional interaction to the computer using new hardware technologies such as cameras, microphones, haptic devices, and olfactory sensors provide efficient, intuitive, and natural communication between human and machine.

There are a few efforts that can be undertaken to improve our prototype system. The current prototype only supports single hand gestures for interaction. Hence, multiple hands gesture interaction can be proposed in order to have more gestures available, reduce the error rate, and ultimately increase the accuracy, speed rate, and user satisfaction, while more hand postures will be selected to support the controlling activities. However, a robust approach in hand gesture recognition is necessary since the multiple hands increase the computational costs and complexity of the system.

The new technology and tools such as Kinect have also added the capabilities of using various media, e.g., multi-array microphone, RGB camera, and laser depth sensor simultaneously. Therefore, employing the two last components of Kinect in gesture recognition will increase robustness in vision-based $\mathrm{HCI}$. Moreover, a combination of gesture recognition, voice recognition, and perhaps haptic feedback will enhance the competency of natural human-computer interaction in a system. Finally, proper usability studies are also required to better understand the effects of multi-modal interactions and how/where they fit. 


\section{Appendix $A$}

\section{Technology in Microsoft Kinect depth camera [65]:}

Kinect was developed on software-based internally by Microsoft Game Studio, and on camera range technology-based by PrimeSense company which developed it through a 3D scanner system called Light Coding. Kinct has a motorized base to direct its sensor toward a desired position. It also has three components - RGB camera, depth sensor, and multi-array microphone - with proprietary software which enables the device to recognize the face/facial gestures, capture full body $3 \mathrm{D}$ motion, and identify voice. The depth sensor includes an infrared laser projector and a monochrome CMOS sensor employed to capture 3D video data under insufficient ambient light conditions. The depth sensing range is adjustable, and a special software automatically calibrates the sensor based on the physical environment.

Microsoft has stated that their Kincet SDK can track up to six persons (as many people as can be fit in camera's field of view in PrimeSense Kinect SDK) simultaneously including two main players, and extract the features of 20 joints per player for skeletal tracking. Some characteristics of Kinect sensor are as following:

- Frame rate of output video $=30 \mathrm{~Hz}$

- Resolution of input video stream for RGB camera $=8$-bit VGA $(640 \times 480)$, Bayer color filter

- Resolution of input video stream for depth sensor $=11$-bit VGA $(640 \times 480)$, monochrome, with 2048 levels of sensitivity

- Practical ranging limit $=1.2 \mathrm{~m}-3.5 \mathrm{~m}$ (with Xbox software)

- Required area to play $=6 \mathrm{~m}^{2}$ 
- Range of tracking $=0.7 \mathrm{~m}-6 \mathrm{~m}$

- Angular field of view $=57^{\circ}$ horizontally, $43^{\circ}$ vertically

- Range of tilting by motorized base $=27^{\circ}$ either up or down

- Field of view and resolution at the minimum distance of $0.8 \mathrm{~m}=87 \mathrm{~cm}$ horizontally, $63 \mathrm{~cm}$ vertically, and $1.3 \mathrm{~mm}$ per pixel

- $\quad$ Microphone array features $=$ four capsules, with $16-$ bit and $16 \mathrm{kHz}$ for each audio channel 


\section{Appendix B}

Comparing Microsoft Kinect SDK and PrimeSense OpenNI SDK [64]:

\section{Microsoft's Kinect SDK (Beta)}

\section{Pro:}

- support for audio

- support for motor/tilt

- full body tracking:

- does not need a calibration pose

- includes head, hands, feet, clavicles

- seems to deal better with occluded joints

- supports multiple sensors

- single no-fuss installer

- SDK has events for when a new Video or new Depth frame is available

\section{Con:}

- licensed for non-commercial use only

- only tracks full body (no mode for hand only tracking)

- does not offer alignment of the color and depth image streams to one another yet

- although there are features to align individual coordinates 
- and there are hints that support may come later

- full body tracking:

- only calculates positions for the joints, not rotations

- only tracks the full body, no upper-body or hands only mode

- seems to consume more CPU power than OpenNI/NITE (not properly benchmarked)

- no gesture recognition system

- no support for the PrimeSense

- only supports Win7 (x86 \& x64)

- no support for Unity3D game engine

- no built in support for record/playback to disk

- no support to stream the raw InfraRed video data

- SDK does not have events for when new user enters frame, leaves frame etc

\section{PrimeSense OpenNI/NITE}

Pro:

- license includes commercial use

- includes a framework for hand tracking

- includes a framework for hand gesture recognition

- can automatically align the depth image stream to the color image

- full body tracking: 
- also calculates rotations for the joints

- support for hands only mode

- seems to consume less CPU power than Microsoft Kinect SDK's tracker (not properly benchmarked)

- also supports the Primesense and the ASUS WAVI Xtion sensors

- supports multiple sensors although setup and enumeration is a bit unusual

- supports Windows (including Vista and XP), Linux and Mac OSX

- comes with code for full support in Unity3D game engine

- support for record/playback to/from disk

- support to stream the raw InfraRed video data

- SDK has events for when new User enters frame, leaves frame etc

\section{Con:}

- no support for audio

- no support for motor/tilt (although we can simultaneously use the CL-NUI motor drivers)

- full body tracking:

- lacks rotations for the head, hands, feet, clavicles

- needs a calibration pose to start tracking (although it can be saved/loaded to/from disk for reuse)

- occluded joints are not estimated 
- supports multiple sensors although setup and enumeration is a bit unusual

- three separate installers and a NITE license string

- SDK does not have events for when new Video or new Depth frames is available 


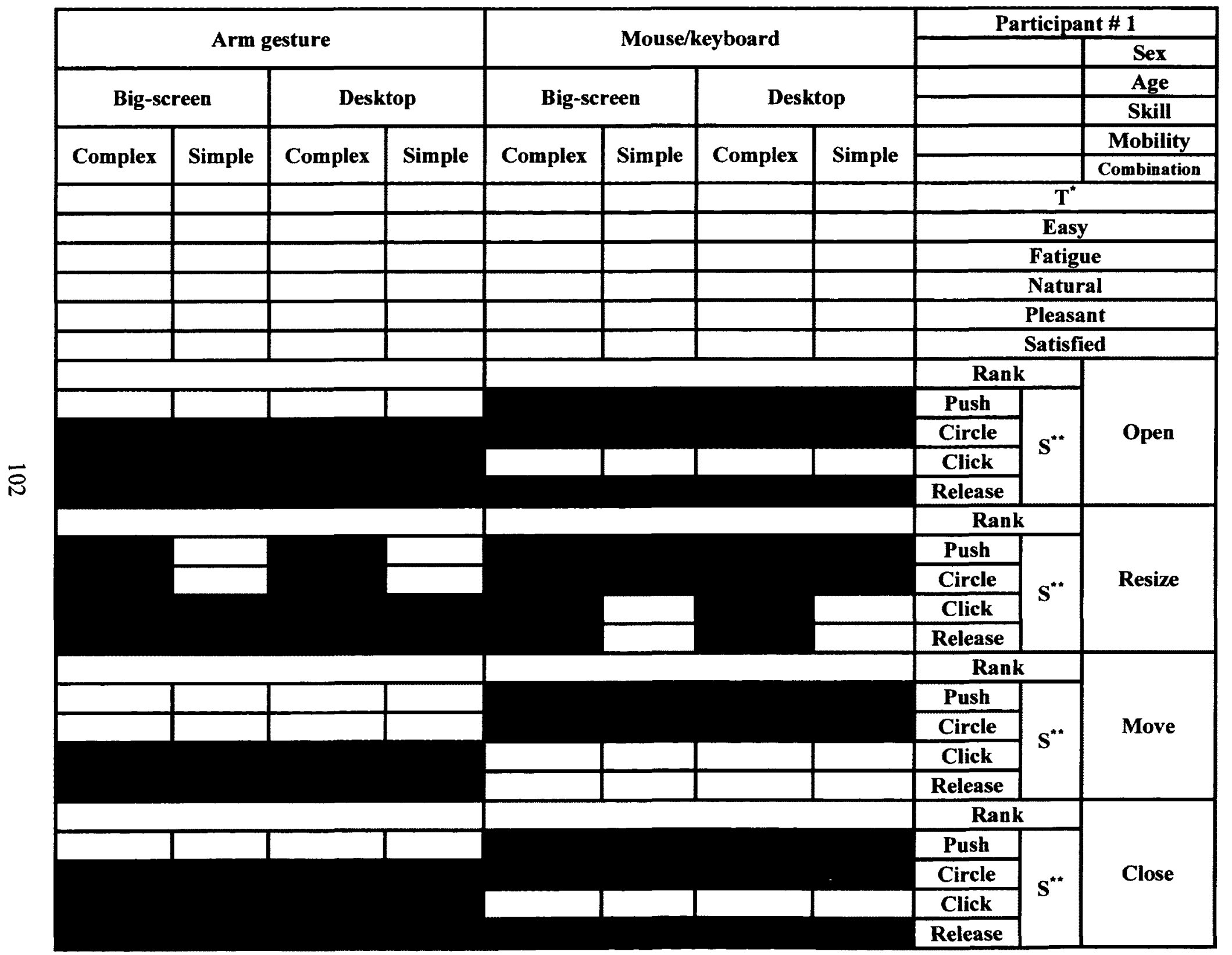

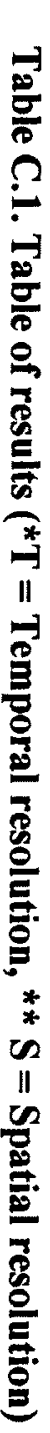




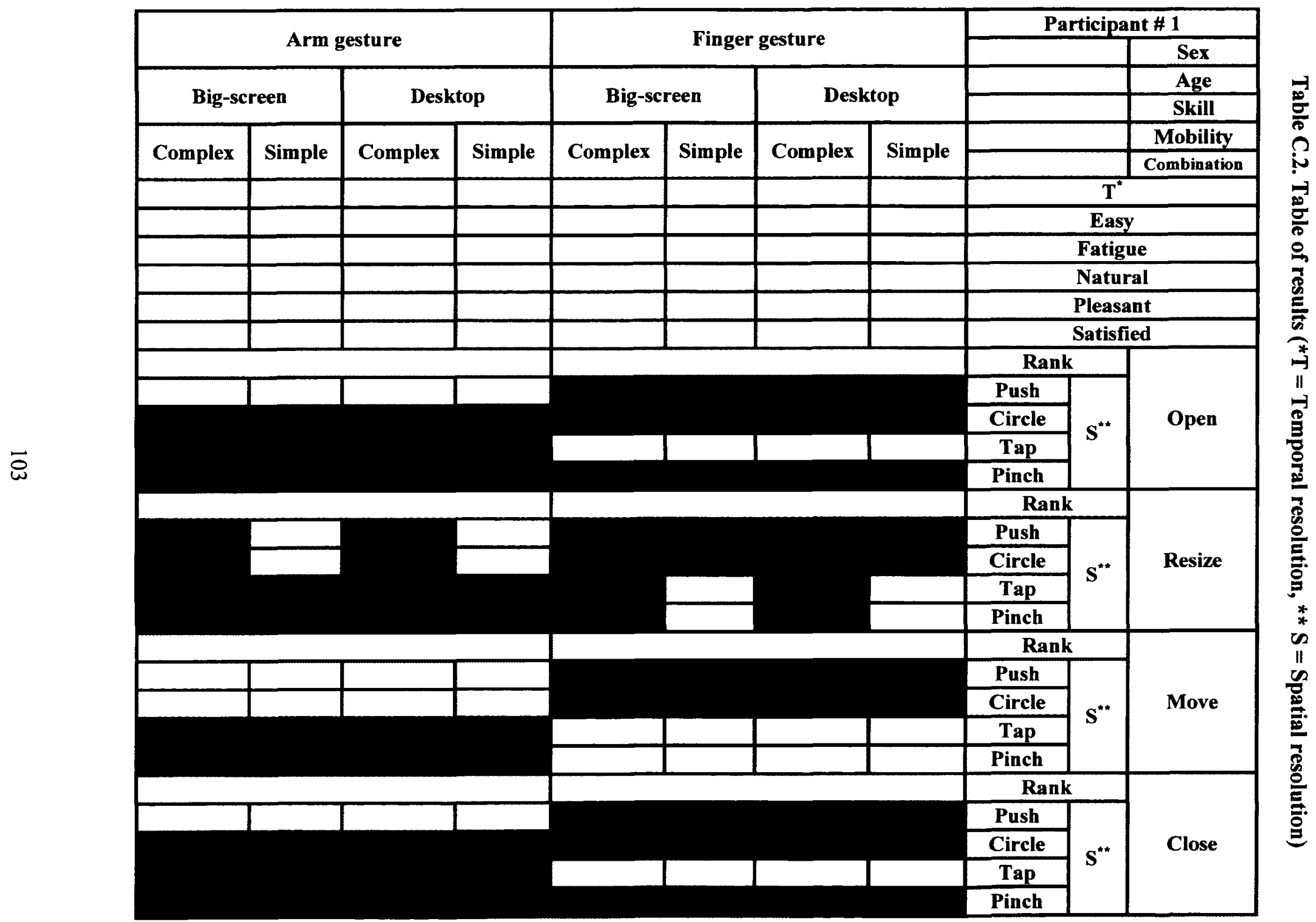




\section{Appendix D}

Extra questions and comments replied by participants:

\section{What they like the most about this hand gesture application:}

- Freedom to move and mobility.

- User has more flexibility.

- Natural, convenient and attractive. It is more natural in comparison to mouse. By getting used to the environment it can be more convenient than a mouse.

- It is a good way for having exercise during work, while this is not possible with mouse.

- The hand gestures "push" for opening and closing an icon or a window, and "circle" for moving or resizing.

- Creates a more immersive experience when interacting with application.

- Probably better for your wrists (carpal tunnel, etc.).

- Advantage of arm gesture application is for users who have disabilities, e.g. not having fingers.

- Arm gesture can enable many applications to be easier.

- Resizing and moving was enjoyable. 
- Interacting alternative to mouse especially at long ranges, e.g. wireless mouse having the same range of operation as gesturing still requires flat surface to move the pointer. Not all flat surfaces comply with laser or track ball mouse.

- The user is kind of detached from the system and can act independently. The arm gesture application increases the flexibility and multi-dimensionality of the user's movements and actions.

- It seems that it can be very helpful for some special purposes. Feasibility seems to be very important factor here.

- It can be very interesting to be applied in seminars, big classrooms and presentations.

- It's more fun and exciting than just using a mouse.

- Multiple possible user inputs.

- Wireless and Intuitive.

- Finger gestures were quite simple as it is a natural gesture to grab with these two fingers and click with the index finger.

- Feasibility, easy to use, pleasurable.

- Natural gestures of fingers and flexibility of the arm.

- Easy to understand the procedure and application.

- Interesting and useful for people who require help. 


\section{What they like the least about this hand gesture application:}

- Initial practice and instructions. Once practice is done, it is very comfortable.

- Hand fatigue, arm gets tired, though this would probably lessen over time. Due to lack of experience you may feel tired and fatigue.

- Hard to close a window. The visibility was not good when using big screen.

- In my view, it would hurt the eyes somehow unless the color and the size of the icons would be changed for a better and clearer distinction. The size of the screen and icons in regards to my distance from the screen. I would rather like a bigger icon size and different colors.

- It is better to be more sensitive like the mouse.

- Pushing with the arm gesture was not pleasant.

- Using the big screen in a grey background, reduce the visibility.

- Jittery nature or pointer on the screen especially during the movement, e.g. making a circle with hand produces something else on the screen.

- Zoom in/out. Use of fingers as oppose to full hand.

- For scaling, a user preferred to do something like stretching and not by drawing circles. 
- Arm fatigue is a big problem because it limits the length of time you can use the application.

- Large open space required for use.

\section{Some other comments:}

- It depends to my needs. I would prefer to have an option of the two applications (mouse and gestures) at the same time, at the same level.

- A user told us to make it easier and more sensitive to close a window, picture or an icon. As the "close" button is small and it is hard to move the pointer precisely to the specified button.

- Mouse is not as fun as wireless (gesture).

- Opening function, needs to press "Enter" key (double-click is more preferred).

- Wireless (gesture) and big-screen is good, but mouse and big-screen is not as comfortable.

- Wireless (gesture) function is fun and innovative, and it is definitely cool and a new way.

- Adding user guide instructions for gesture to make it effortless, e.g. fixing elbow.

- After practice, gesture is much easier.

- Remote (gesture) with big-screen is the most fun. 
- Instructions with user guide helped a lot.

- A participant suggests adding more functionality to both desktop interface and hand gesture.

- Another participant would like to see an option to active or de-active the pointer tracking due to hands fatigue. He would like to adjust his hand position after deactivating the pointer (like mouse pad).

- Some of the participants told us that the application needs to have more options, e.g. menus.

- Instead of circle motions, either a push to "grab" a window (for moving or resizing), or actually recognizing a grab or pinch gesture would be more intuitive.

- It could be nicer if the pointer on the big-screen was somehow different and kind of matching with our action, e.g. when we want to move an icon, the pointer was like two fingers trying to grab the icon and move it.

- People that use mouse and keyboard for a long time may get disordered for overusing, so arm gesture can solve this problem.

- If you put extra movement for dropping, it would be easier, e.g. suppose by moving hand backward, you drop the icon.

- Most students have not used such an application before, so by practicing it would become easier for them. 
- It is much better if you improve the push button feature in gesture. When we want to draw a circle, it is nice to have a sound recognition and before starting to draw a circle be able to say the word "shape" and then a line appear on the screen and follow the pointer. The benefit of this functionality is to help you better draw the shape.

- Stability of pointer should be better.

- Finger gestures in combination with hand gesture and multiple fingers operation is recommended.

- Maybe larger icons on the big screen and different color for each icon.

- The color and the size of icons should be selected in a way that they would be easy to be pinpointed by the eyes. It would be preferred to use fingers instead of a hand only. Using both hands and fingers simultaneously would also increase the feasibility of the actions.

- Need to be more sensitive, gripping tools be more intuitive, so arms gets tired, and hyper extension and overreaching painful, it should respond to smaller movements so arms do not get tired/reaching. Haptic feedback, to know if user had clicked something. Better to catch both hands movements instead of having to transfer.

- It can be a lot easier if fingers can be used instead of the whole hand and it would get more time efficient with the fingers involved.

- Right-click capability. 
- Gestures for "Shift", "Control" and "Alt" buttons.

- Improve the user interface design.

- Must have: capability to use mouse upon request. Nice to have: capability to switch arm/fingers based on user preference.

- Would be simpler and most natural to use fisting for the arm gestures to grab. Resizing should be done using any four corners. 


\section{Appendix $E$}

The internal process of gesture recognition through a $\mathrm{HCl}$ application includes the following steps [83]:

- Look at how they are created from a sensor

- How to filter out the noise and disturbances to enhance features

- How to calibrate cameras and sensors to improve accuracy

- How to estimate registration between data seen from various views

- How to extract information from images (e.g. edges, contours, lines, objects)

- How to segment images in each of its components (e.g. objects)

- How to recognize specific objects and estimate their properties

- How to compute $3 D$ information from $2 D$ intensity images (e.g. stereoscopic vision, structured lighting, shape from shading)

- How to detect and represent movement in images

- How to track moving objects in images

- How to build virtual representations from images (e.g. models)

- How to apply imaging to robotic and autonomous systems (e.g. quality inspection, pose estimation, visual feedback, path planning, automatic surveillance, biometric recognition, etc.) 


\section{Theoretical Support}

In this section, to elaborate the above mentioned internal steps, we review some existing theoretical details [83] that we have used directly/indirectly in our prototype design.

\section{Perspective Projection}

Using the pinhole camera model, simple equations can be derived to represent the projection of an object surface point on the image plane.
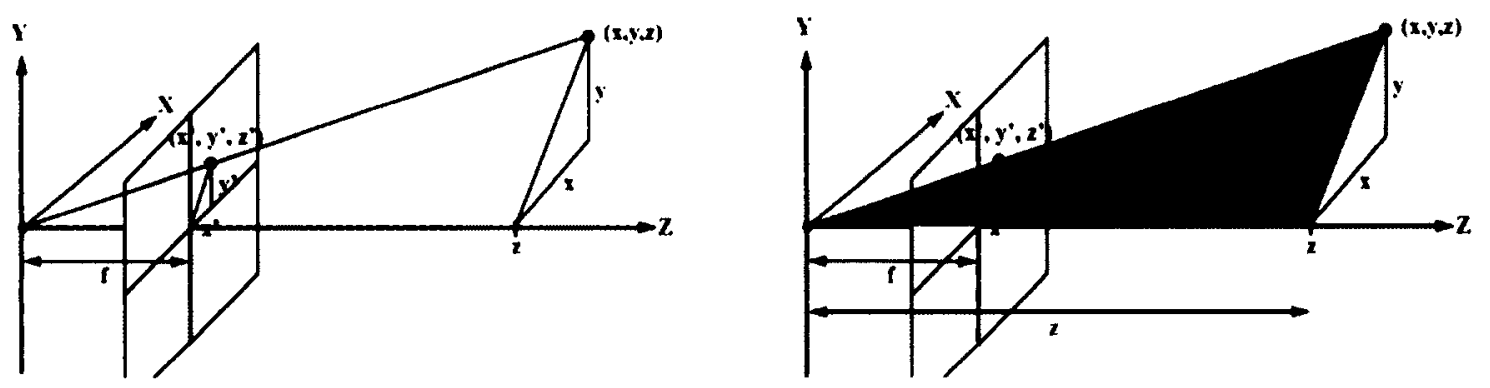

Figure E.1. Perspective projection.

$$
\frac{f}{z}=\frac{r^{\prime}}{r}
$$

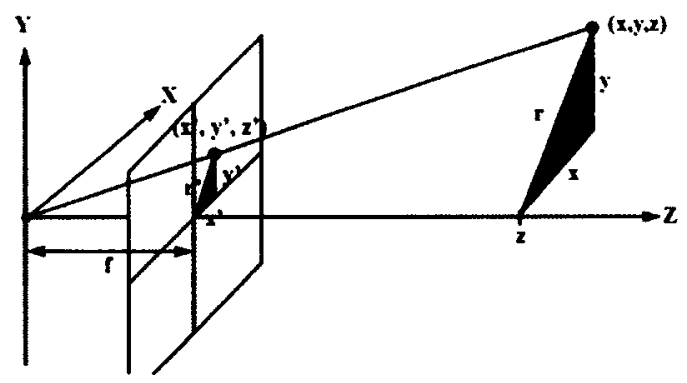

Figure E.2. Image and scene planes.

$$
r^{\prime}=\sqrt{\left(x^{\prime}\right)^{2}+\left(y^{\prime}\right)^{2}} \quad, \quad r=\sqrt{x^{2}+y^{2}}
$$




$$
\frac{x \prime}{x}=\frac{y \prime}{y}=\frac{r \prime}{r}
$$

Combining our equations from similar triangles:

$$
\frac{x \prime}{x}=\frac{y^{\prime}}{y}=\frac{r \prime}{r}=\frac{f}{z}
$$

We get the direct perspective projection equations:

$$
x^{\prime}=\frac{f}{z} x \quad, \quad y^{\prime}=\frac{f}{z} y
$$

And on the image plane, the $z^{\prime}$ coordinate is always:

$$
z^{\prime}=f
$$

The direct perspective projection equations can be rewritten as follow:

$$
\frac{x}{x \prime}=\frac{z}{f}=\frac{y}{y \prime}
$$

Placing $(x, y, z)$ in evidence, we get the inverse perspective projection equations:

$$
x=\frac{z}{f} x^{\prime} \quad, \quad y=\frac{z}{f} y^{\prime} \quad, \quad z=\frac{f}{y^{\prime}} y=\frac{f}{x^{\prime}} x
$$

Unfortunately, with the direct and inverse perspective projection equations the position of the image point has a nonlinear relationship with the coordinates $(x, y)$ of the object surface point (it depends on both $\mathrm{f}$ and $\mathrm{z}$ ).

\section{Weak Perspective Camera}

In order to linearize the relationship between the coordinates of the image point and the object surface point, we can make the assumption that the distance between two points in the scene is much smaller than the average distance, $\overline{\mathbf{z}}$, between the object and the camera. This implies that the shape of the object is relatively smooth. In this case, we approximate the perspective projection by the following: 


$$
x^{\prime} \approx \frac{f}{\bar{z}} x \quad, \quad y^{\prime} \approx \frac{f}{\bar{z}} y
$$

As $\overline{\mathbf{z}}$ is now a constant, we obtain a linear relationship between the image point and the scene point that depends only on the focal length.

\section{Perspective Projection as a Homogeneous Transformation}

We consider the coordinates of the image point, $\left(\boldsymbol{x}^{\prime}, \boldsymbol{y}^{\prime}, \mathbf{z}^{\prime}\right)$, as an homogeneous coordinate vector, $\left[\widetilde{\boldsymbol{x}}^{\prime}, \widetilde{\boldsymbol{y}}^{\prime}, \tilde{\boldsymbol{z}}^{\prime}, \widetilde{\boldsymbol{w}}^{\prime}\right]^{T}$. The coordinates of the object surface point, $(\boldsymbol{x}, \boldsymbol{y}, \mathbf{z})$, can also be written as an homogeneous coordinate vector, $[\widetilde{\boldsymbol{x}}, \widetilde{\boldsymbol{y}}, \tilde{z}, \widetilde{w}]^{T}$, where the weight, $\widetilde{\boldsymbol{w}}$, is equal to 1 as this point is a coordinate point in real 3D space. We can represent the perspective projection as following:

$$
\left[\begin{array}{c}
\widetilde{x}^{\prime} \\
\widetilde{y}^{\prime} \\
\widetilde{z}^{\prime} \\
\widetilde{w}^{\prime}
\end{array}\right]=P\left[\begin{array}{l}
\widetilde{x} \\
\widetilde{y} \\
\tilde{z} \\
1
\end{array}\right]=\left[\begin{array}{llll}
1 & 0 & 0 & 0 \\
0 & 1 & 0 & 0 \\
0 & 0 & 1 & 0 \\
0 & 0 & \frac{1}{f} & 0
\end{array}\right]\left[\begin{array}{c}
\widetilde{x} \\
\widetilde{y} \\
\tilde{z} \\
1
\end{array}\right]
$$

This matrix product leads to:

$$
\left[\begin{array}{c}
\widetilde{x}^{\prime} \\
\widetilde{y}^{\prime} \\
\tilde{z}^{\prime} \\
\widetilde{w}^{\prime}
\end{array}\right]=\left[\begin{array}{c}
\tilde{\boldsymbol{x}} \\
\tilde{\boldsymbol{y}} \\
\tilde{\boldsymbol{z}} \\
\tilde{\boldsymbol{z}} / f
\end{array}\right]
$$

We note that the weight of the projected point, $\widetilde{w}^{\prime}$, is not equal to 1 as this is not a coordinate point in 3D space. The projection operation introduces some distortion in world representation (3D on 2D) that appears as a scaling factor. Ultimately, we get:

$$
\begin{aligned}
& \tilde{x}^{\prime}=\frac{x}{(\tilde{z} / f)}=\frac{f x}{\tilde{z}} \\
& \tilde{y}^{\prime}=\frac{y}{(\tilde{z} / f)}=\frac{f y}{\tilde{z}}
\end{aligned}
$$




$$
\tilde{z}^{\prime}=\frac{z}{(\tilde{z} / f)}=\frac{f z}{\tilde{z}}=f
$$

Therefore, the direct perspective projection operation can be defined as a $4 \times 4$ matrix $P$ :

$$
P=\left[\begin{array}{llll}
1 & 0 & 0 & 0 \\
0 & 1 & 0 & 0 \\
0 & 0 & 1 & 0 \\
0 & 0 & \frac{1}{f} & 0
\end{array}\right]
$$

This matrix cannot be inverted as its determinant equals zero. The definition of the projection matrix $P$ also depends on where the origin of the reference frame $(0,0,0)$ is located.

\section{Perspective Projection with Multiple Reference Frames}

Assuming a calibrated camera (to find out where are the center of projection and image plane with respect to the casting), we need to use two reference frames:

- One reference frame with respect to which image points are defined (camera centered frame).

- One global reference frame with respect to which everything (including the camera reference frame) is defined.

Assuming that we know where is the camera with respect to the global reference frame. These two frames and the relationships between them are illustrated by the means of a transformation graph as shown in Figure E.3. 


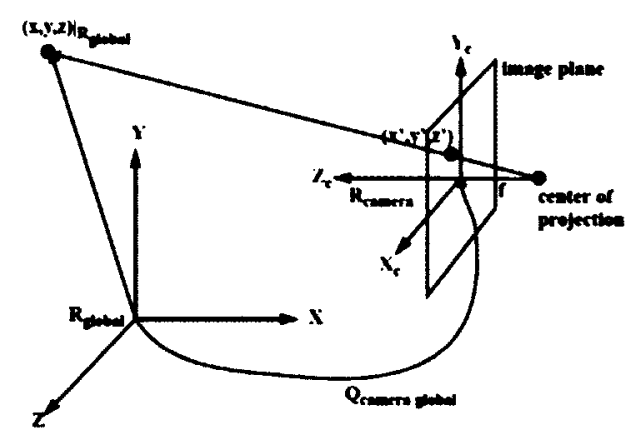

Figure E.3. Relationships between camera and global reference frames.

To complete the coordinates of the image point, $\left(x^{\prime}, y^{\prime}, z^{\prime}\right)$, one must first find out the coordinates of this point with respect to the camera reference frame and then apply the projection operation.

$$
\left.\left(x_{c}, y_{c}, z_{c}\right)\right|_{R_{c a m e r a}}
$$

And then apply the projection operation:

$$
\widetilde{v}^{\prime}=P \widetilde{v}_{\text {camera }}=P Q_{\text {camera } / \text { global }}^{-1} \widetilde{v}_{\text {global }}
$$

Where:

$\widetilde{\boldsymbol{v}}^{\prime}=\left(\widetilde{\boldsymbol{x}}^{\prime}, \widetilde{\boldsymbol{y}}^{\prime}, \tilde{\mathbf{z}}^{\prime}, \widetilde{\boldsymbol{w}}^{\prime}\right)$ is the image point, $\widetilde{\boldsymbol{v}}_{\text {camera }}=\left(\widetilde{\boldsymbol{x}}_{c}, \widetilde{\boldsymbol{y}}_{c}, \tilde{\mathbf{z}}_{c}, \mathbf{1}\right)$ is the object point w/r to $\boldsymbol{R}_{\text {camera }}$, and $\widetilde{\boldsymbol{v}}_{\text {global }}=(\widetilde{\boldsymbol{x}}, \widetilde{\boldsymbol{y}}, \tilde{\mathbf{z}}, 1)$ is the object point $\mathrm{w} / \mathrm{r}$ to $\boldsymbol{R}_{\text {global }}$.

\section{Motion}

Assuming that the camera is located at the origin of the reference frame, each image is represented by a matrix of intensity pixels:

$$
I(x, y, t)
$$

It is important that we know the time, $t$, at which the image has been collected in order to eventually estimate the magnitude of the movement (flow of movement). 
Because we are working with only $2 \mathrm{D}$ projections (orthographic or perspective), only the characteristics of a motion restricted to a plane, that is parallel to the image plane, can be estimated quantitatively.

Generic 3D motion can be detected but estimated only quantitatively.

As motion analysis is usually based on intensity variation, any illustration change in a scene has the same effect as moving objects.

\section{Motion Detection}

The simplest procedure to detect motion between two or more successive frames is to compute the difference in the intensity level of corresponding pixels between these images.

A simple detection algorithm looks like:

$$
\text { Motion }=\left\{\begin{array}{lr}
1, \text { if }\left|I\left(x, y, t_{1}\right)-I\left(x, y, t_{2}\right)\right|>\text { threshold } \\
0, & \text { Otherwise }
\end{array}\right.
$$

The result is a motion image where ' 1 ' pixels correspond to moving points in the scene while ' 0 ' pixels are fixed points.

A proper registration between images is required for this approach to be reliable.

This algorithm is highly sensitive to noise in images and to illumination variations between $t_{1}$ and $t_{2}$.

In general, more ' 1 ' pixels appear in the resulting image than the number of actually moving points in the scene, create ghost effects.

More robust techniques for motion detection examine the variation in local intensity distribution rather than pixel-based differences.

Pixels are first grouped into small non-overlapping clusters. 
Next, the average and variance of intensity level of pixels in each cluster is computed:

$$
\begin{gathered}
\mu_{t}=\frac{1}{N} \sum_{j=1}^{N} I_{j}(t) \\
\sigma_{t}^{2}=\frac{1}{N} \sum_{j=1}^{N}\left(I_{j}(t)-\mu_{t}\right)^{2}
\end{gathered}
$$

A variation function between two successive frames is computed:

$$
\lambda=\frac{\left[\frac{\sigma_{1}^{2}+\sigma_{2}^{2}}{2}+\left(\frac{\mu_{1}-\mu_{2}}{2}\right)^{2}\right]^{2}}{\sigma_{1}^{2} \cdot \sigma_{2}^{2}}
$$

Finally, the motion detection is completed by a thresholding operation:

$$
\text { Motion }=\left\{\begin{array}{lr}
1, & \text { if } \lambda>\text { threshold } \\
0, & \text { Otherwise }
\end{array}\right.
$$

\section{Motion and Segmentation}

A scene in which some objects are moving can be segmented into subparts. Each subpart is associated with a specific movement, usually corresponding to one object or a group of objects having the same behaviour. A stationary region includes the background (for a fixed camera) and all fixed objects. As with static scene, segmentation of moving scene can be based on edge detection. However, intensity changes that result in edges, so called moving edges, now depend both on spatial variations and on temporal variations. Moving edges can be detected by a combination of the temporal and the spatial gradients. The spatial gradient is defined as for static images.

$$
G(I(x, y))=\left[\begin{array}{l}
G_{x} \\
G_{y}
\end{array}\right]=\left[\begin{array}{l}
\frac{d I}{d x} \\
\frac{d I}{d y}
\end{array}\right]
$$

With: 


$$
|G(I(x, y))|=\sqrt{G_{x}^{2}+G_{y}^{2}} \quad, \quad \alpha(I(x, y))=\operatorname{atan}\left(\frac{G_{y}}{G_{x}}\right)
$$

The temporal gradient is:

$$
G(I(t))=G_{t}=\frac{d I}{d t}
$$

Combining both components:

$$
G(I(x, y, t))=G(I(x, y)) \cdot G(I(t))
$$

The product of the two gradient behaves like an AND operator. Conventional edge detectors can be used to compute the spatial gradient, e.g. Roberts, Sobel, Canny (adaptive threshold on this research).

A difference operator is used to compute the temporal gradient:

$$
\text { Motion }=\left\{\begin{array}{lr}
1, & \text { if }\left|I\left(x, y, t_{1}\right)-I\left(x, y, t_{2}\right)\right|>\text { threshold } \\
0, & \text { Otherwise }
\end{array}\right.
$$

Once moving edges are detected, they can be grouped to delimitate the contours of moving objects.

\section{Tracking}

In some applications, objects must be tracked over a sequence of frames. When a single moving object is involved, it is relatively easy to detect it in each frame and then to estimate its relative displacement. When more than one object is moving, partial recognition of objects is required to determine which part of the image the tracker must be applied to. Recognition of objects is a complex task usually based on feature extraction that is lengthy and not fully reliable. Also the tracking problem can be simplified by applying the concept of trajectories. 


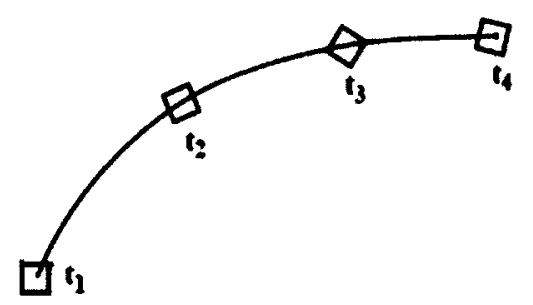

Figure E.4. A trajectory is a virtual or mathematical encoding of a series of positions and orientations that an object visits over the time.

Assuming that objects move in a smooth way, the relative displacement between successive frames should not be very large. Moreover, due to inertia, the motion of an object cannot change instantaneously. Therefore, their movement can often be modeled by simple equations that represent their displacement over the time.

If the trajectory of an object is represented by:

$$
T_{j}=\left[X_{i 1}, X_{i 2}, \ldots, X_{i n}\right]
$$

The tracking is based on the assumption that the object should stay close to this trajectory. Then a division function can be computed to facilitate the search of the object in the next few frames based on past localizations.

$$
d_{i}^{k}=f\left(\overline{X_{l(k-1)} X_{l(k)}}, \overline{X_{l(k)} X_{I(k+1)}}\right)
$$

The correspondence problem between the previous frame and the new one can be solved by minimizing this deviation function.

If many objects are moving and need to be tracked, a combination of such deviation functions need to be defined but matching is much more difficult and objects tend to be mixed one with another.

Occlusions (partial or complete) also remain an important problem. 


\section{Segmentation from Contours}

If the segmentation is to be based on pixels lying on region boundaries, an edge detection step can be applied first. Neighbour edge pixels are then connected together to create contour (on a pixel by pixel basis or with curve fitting).

Once the contours are discovered, attempts are made to create a close contour representation for object.

In practice, contours are rarely complete and can hardly be connected one to each other to create a close contour. As a result, image regions or segments are an approximation of the actual object projection on the image plane. This approximation in the object representation introduces errors in the object properties estimation that have an important impact in any subsequent classification and recognition task.

\section{Active Triangulation}

Triangulation is a widely used approach in the estimation of distance with sensors. Triangulation is used under different forms by active range sensors. This system requires its own source of light (forming a specific pattern on the object), and often the light source is laser. The light source is located at a distance ' $b$ ' (called the baseline) from the center of projection of the camera. A CCD intensity camera is used to collect images from which the projection of the light pattern is analyzed to estimate the distance. 


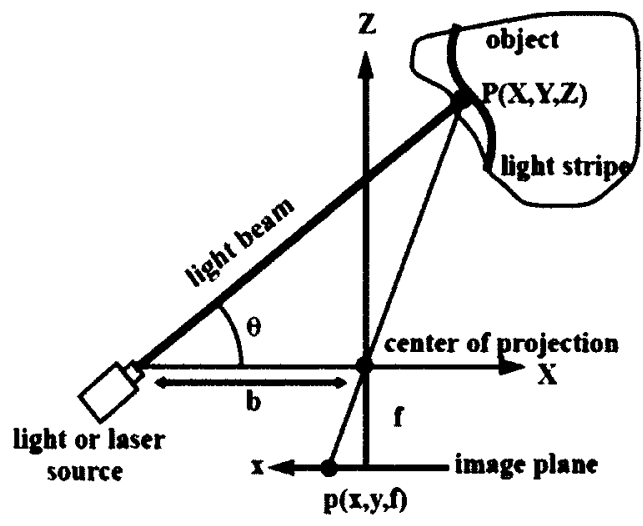

Figure E.5. Active triangulation.

From the figure we can write:

$$
\tan \theta=\frac{z}{b+X}
$$

Using the direct perspective projection equations:

$$
\begin{aligned}
& x=\frac{f}{Z} X \\
& y=\frac{f}{Z} Y
\end{aligned}
$$

Combining the first and the second equations:

$$
\tan \theta=\frac{Z}{b+\frac{x Z}{f}}=\frac{Z}{\frac{b f+x Z}{f}}=\frac{f Z}{b f+x Z}
$$

That we can rewrite as:

$$
b f \cdot \tan \theta+x Z \cdot \tan \theta=f Z
$$

Placing $\mathrm{Z}$ in evidence:

$$
\begin{gathered}
b f \cdot \tan \theta=f Z-x Z \cdot \tan \theta=Z(f-x \cdot \tan \theta) \\
Z=\frac{b f \cdot \tan \theta}{f-x \cdot \tan \theta}=\frac{b f}{\frac{f}{\tan \theta}-x}=\frac{b f}{f \cdot \cot \theta-x}
\end{gathered}
$$


Replacing $\mathrm{X}$ and $\mathrm{Y}$ with this expression for $\mathrm{Z}$ in the first and second inverse perspective projection equations:

$$
\begin{aligned}
& X=\frac{x Z}{f}=\frac{x b f}{f(f \cdot \cot \theta-x)}=\frac{b}{f \cdot \cot \theta-x} \cdot x \\
& Y=\frac{y Z}{f}=\frac{y b f}{f(f \cdot \cot \theta-x)}=\frac{b}{f \cdot \cot \theta-x} \cdot y
\end{aligned}
$$

Therefore, we can estimate the position of a point $P(X, Y, Z)$ on the object surface as a function of the position of its image on the image plane $p(x, y, f)$.

$$
\left[\begin{array}{l}
X \\
Y \\
Z
\end{array}\right]=\frac{b}{f \cdot \cot \theta-x}\left[\begin{array}{l}
x \\
y \\
f
\end{array}\right]
$$




\section{Appendix $F$}

User satisfaction data in arm-mouse experiment (average ranking for all 20 participants) are shown in the following tables:

Table F.1. Questions for simple/mouse/desktop.

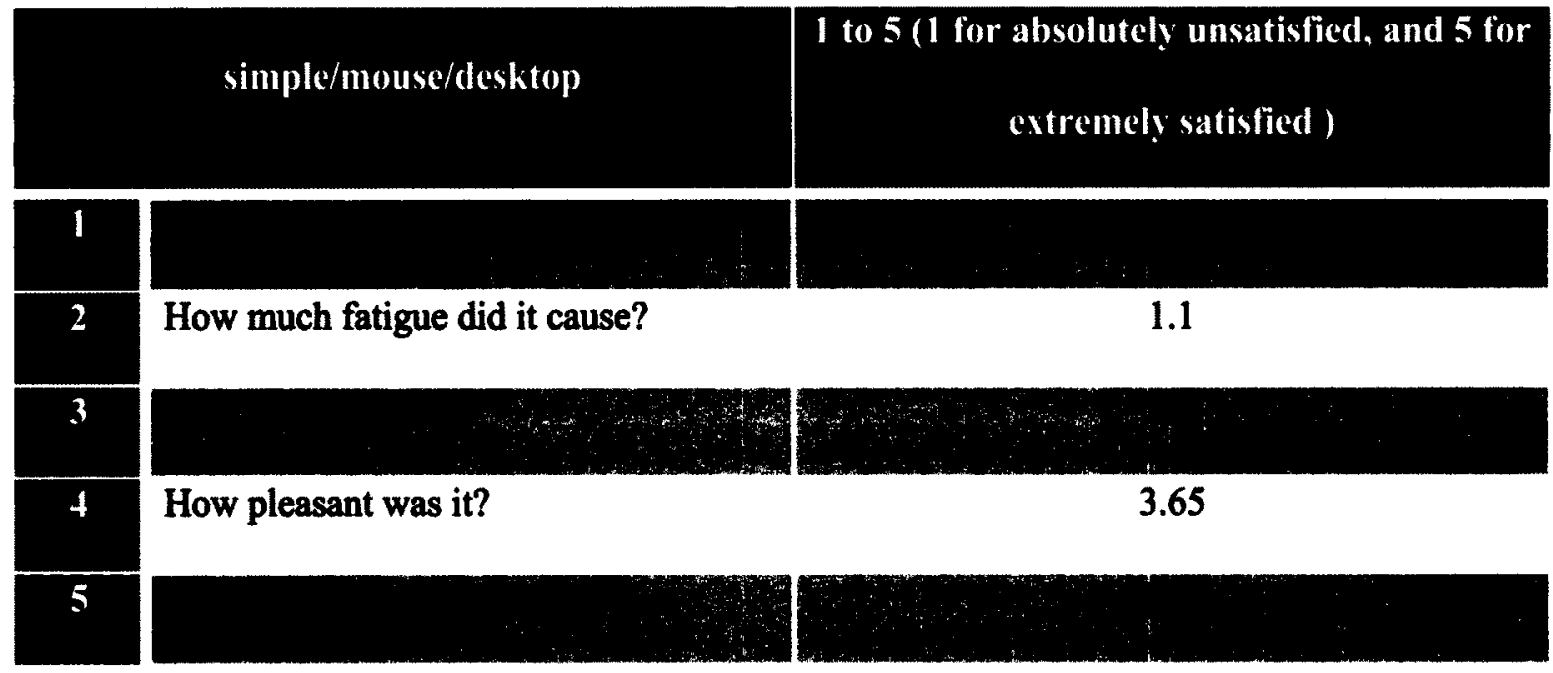

Table F.2. Questions for simple/mouse/big-screen.

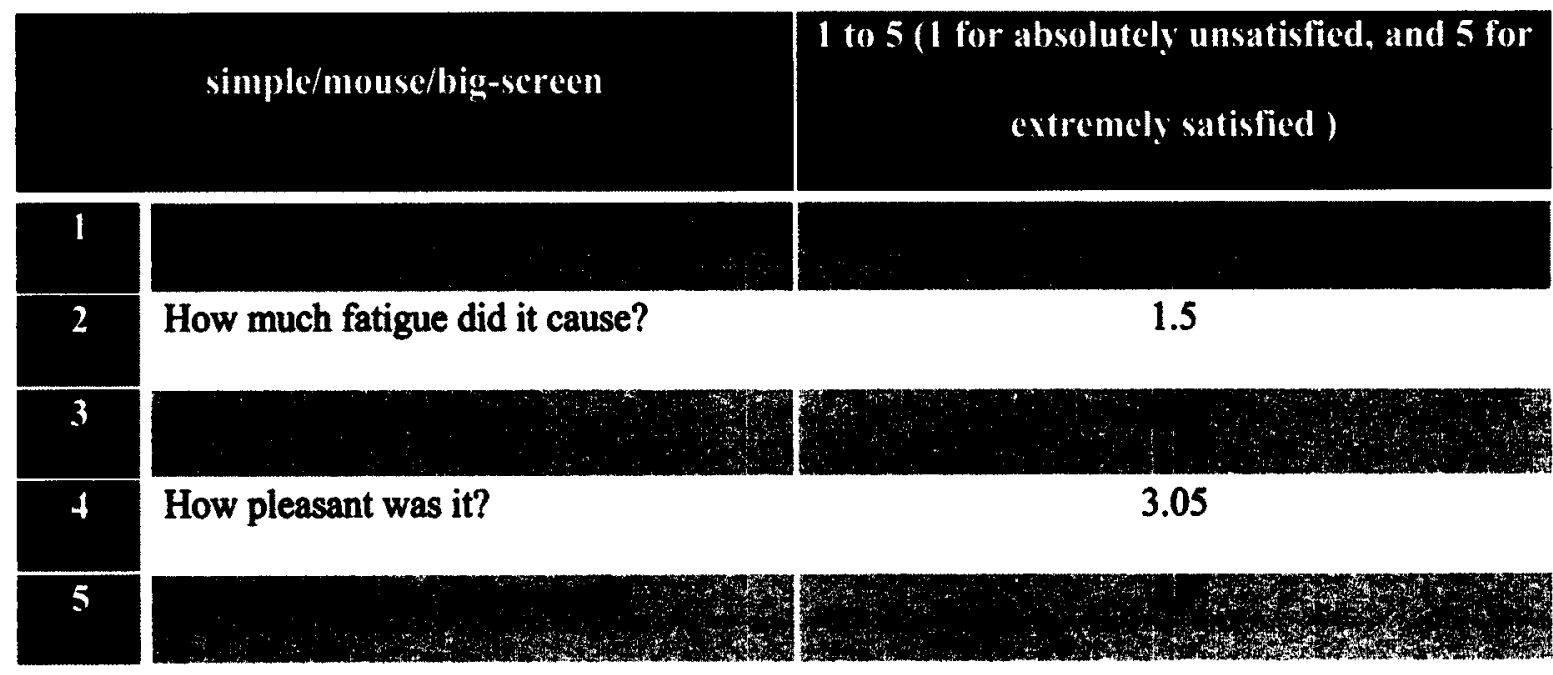


Table F.3. Questions for simple/gesture/desktop.

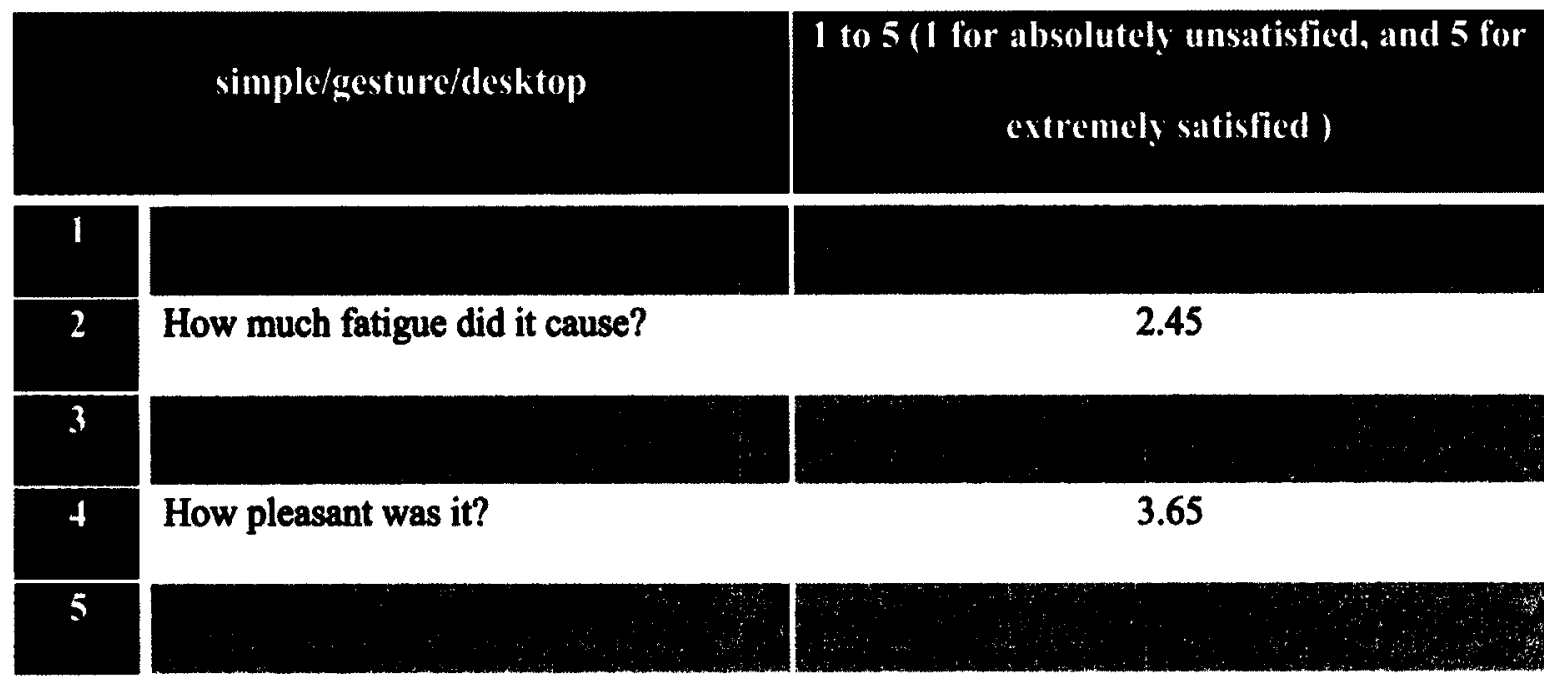

Table F.4. Questions for simple/gesture/big-screen.

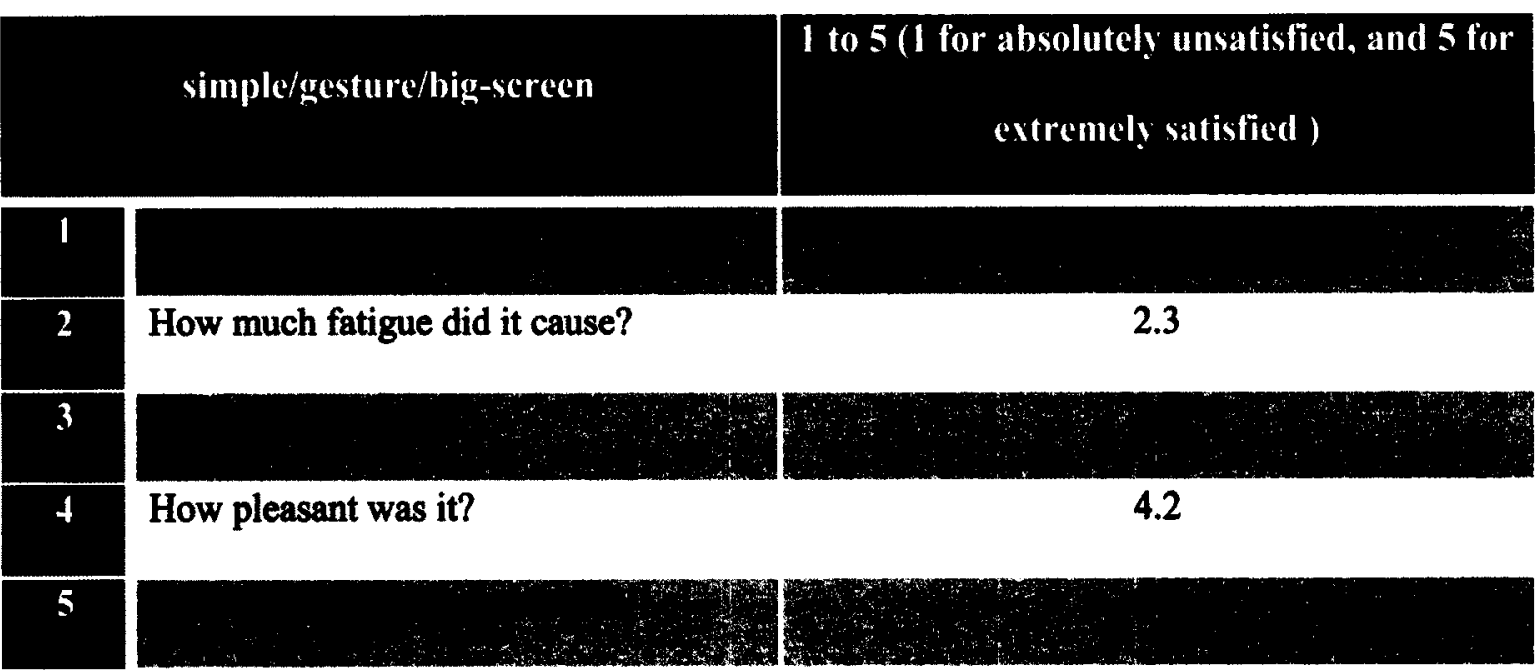


Table F.5. Questions for complex/mouse/desktop.

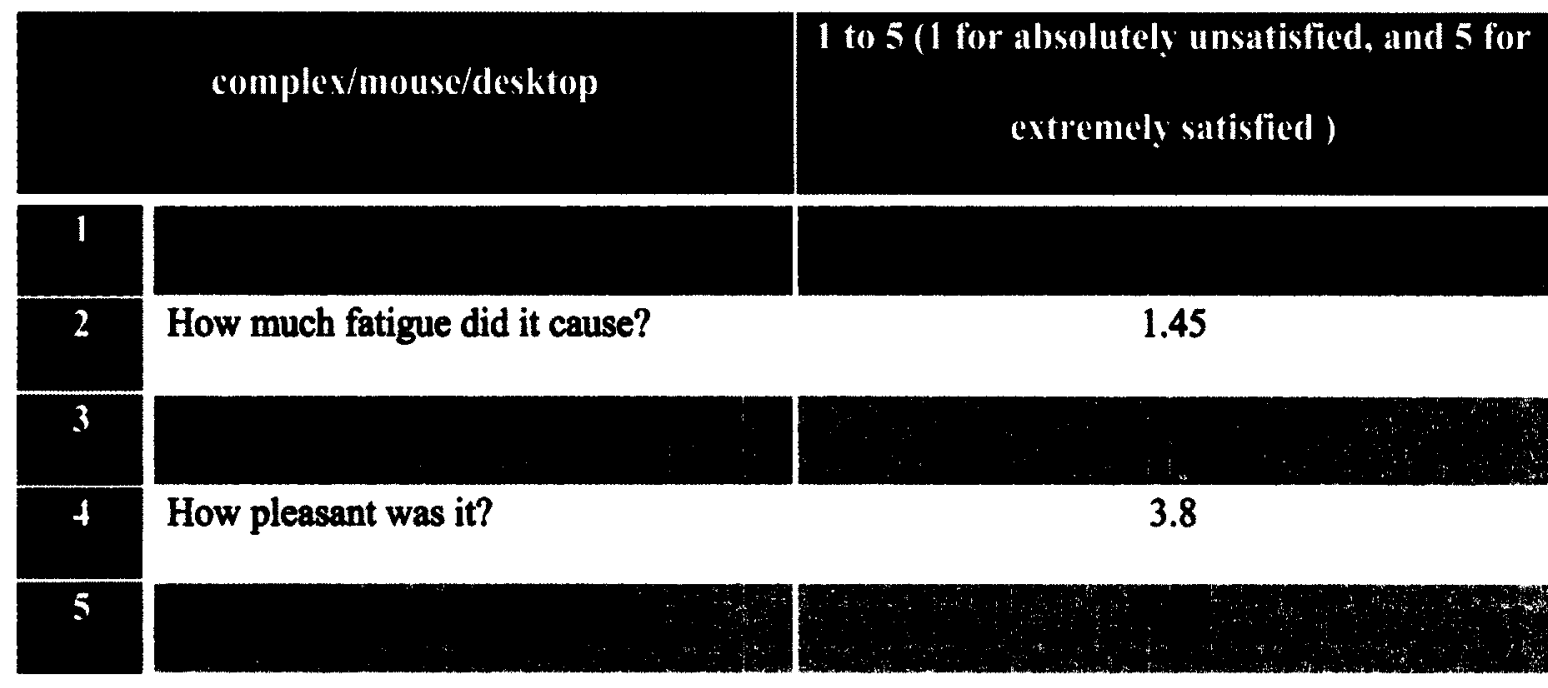

Table F.6. Questions for complex/mouse/big-screen.

complex/mouse/big-screen

1 to 5 ( 1 for absolutely unsatisfied, and 5 for extremely satisfied )

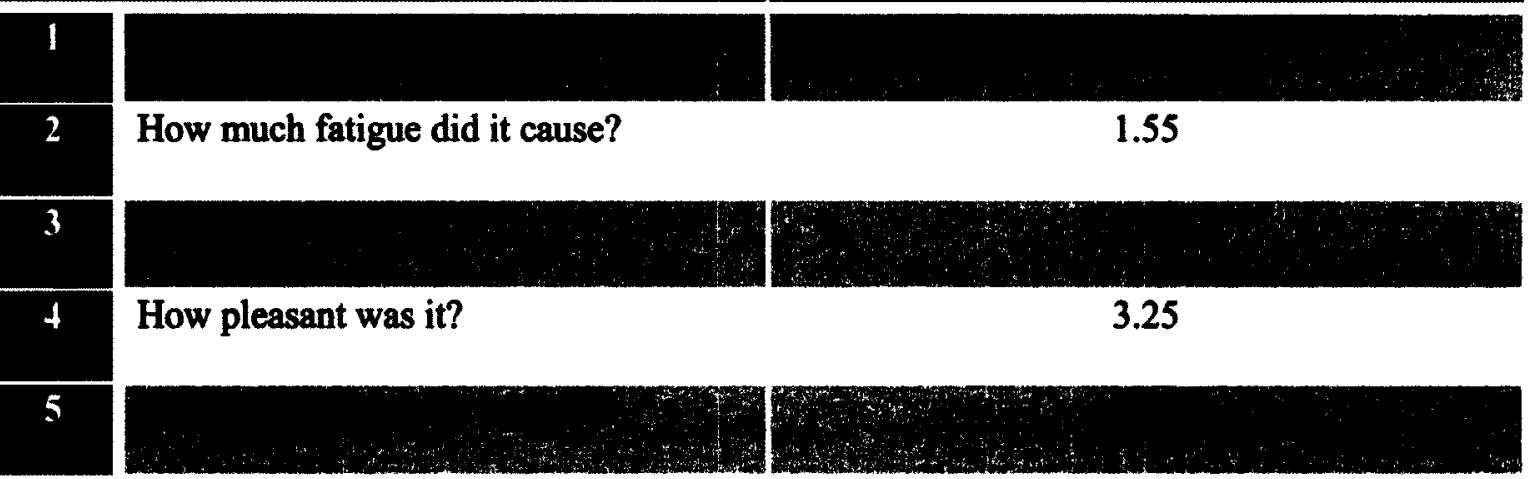


Table F.7. Questions for complex/gesture/desktop.

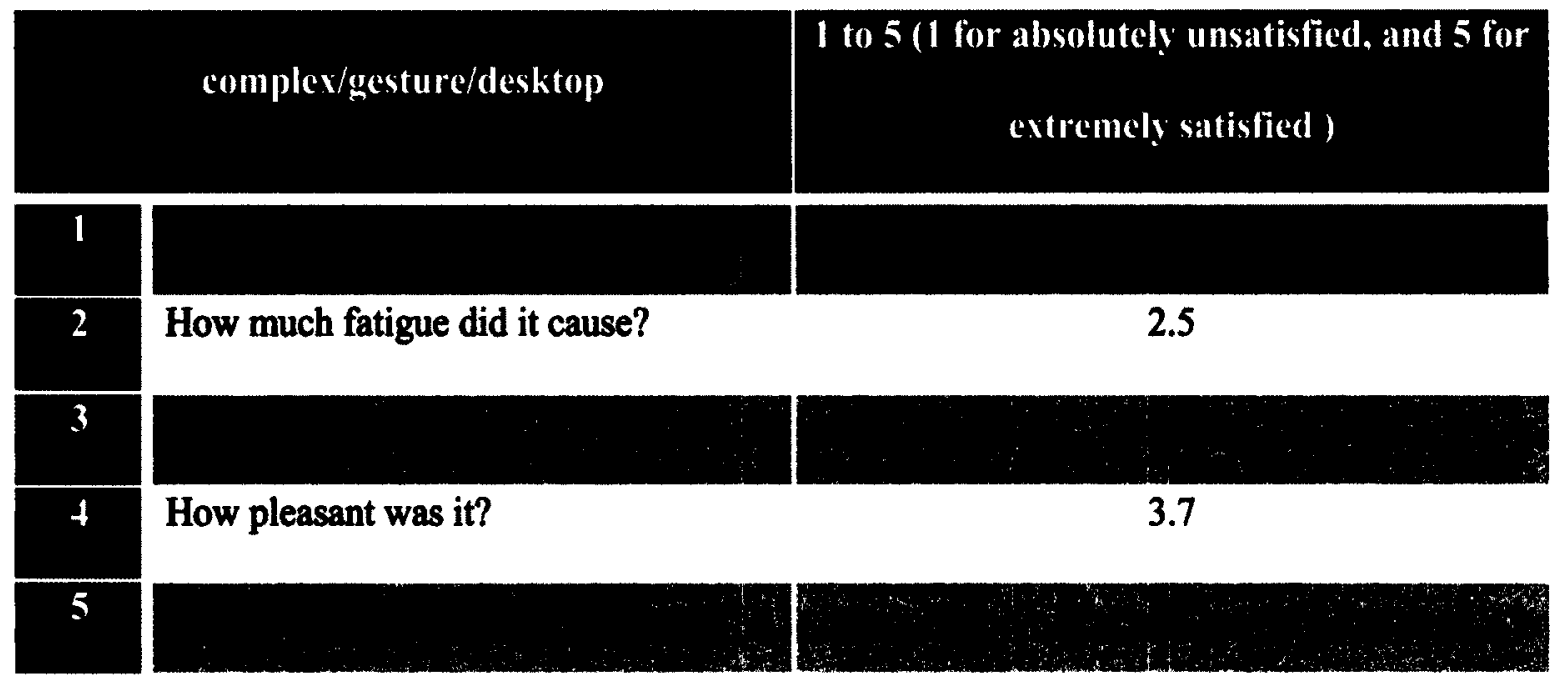

Table F.8. Questions for complex/gesture/big-screen.

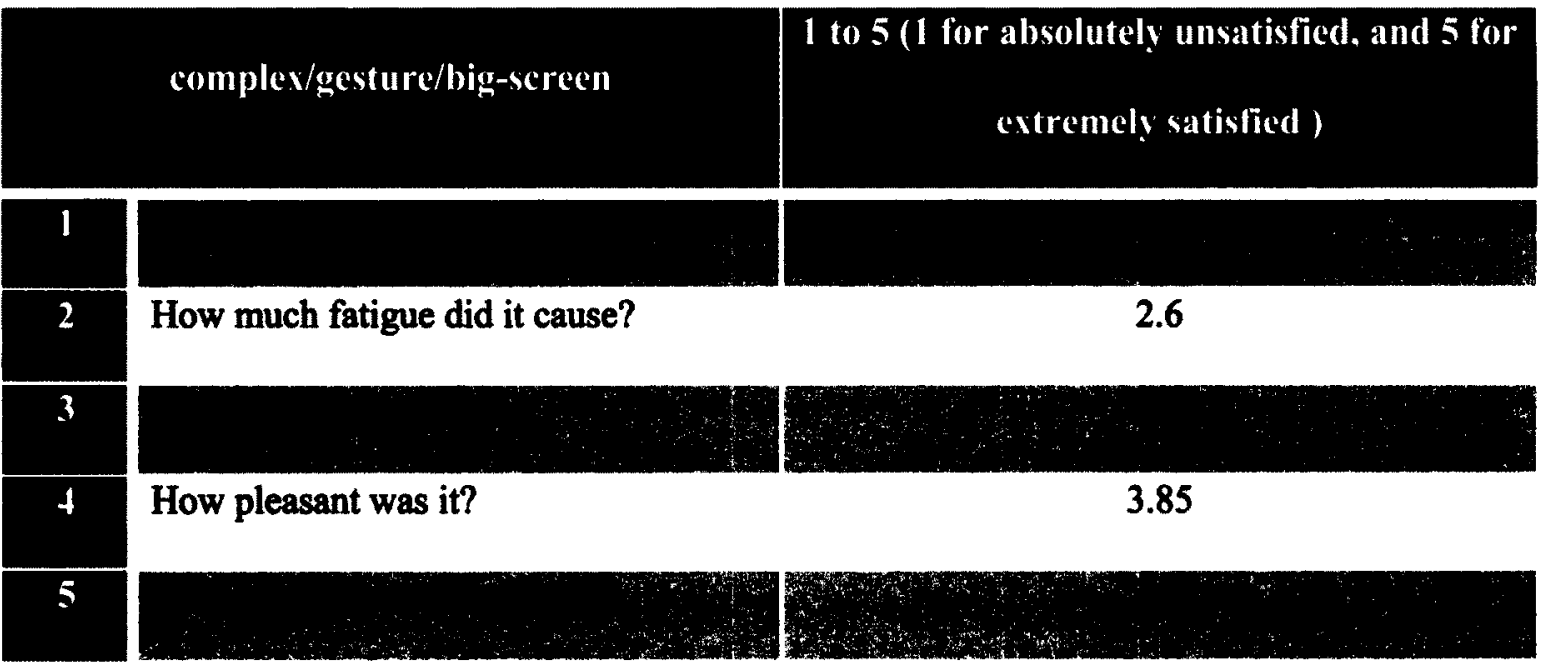


Table F.9. User satisfaction for primitive tasks.

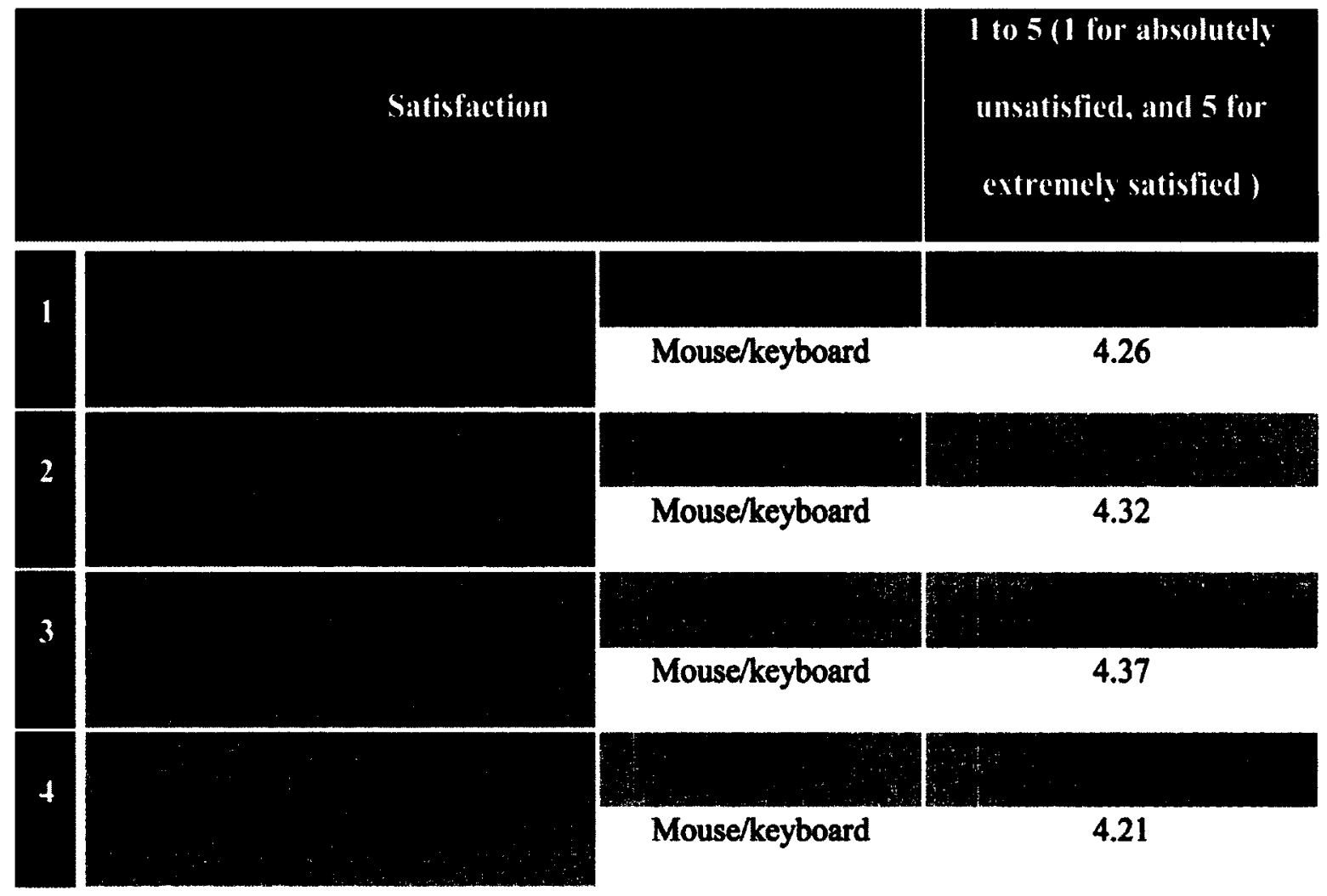

User satisfaction data in arm-finger experiment (average ranking for all 10 participants) are shown in the following tables:

Table F.10. Questions for simple/finger/big-screen.

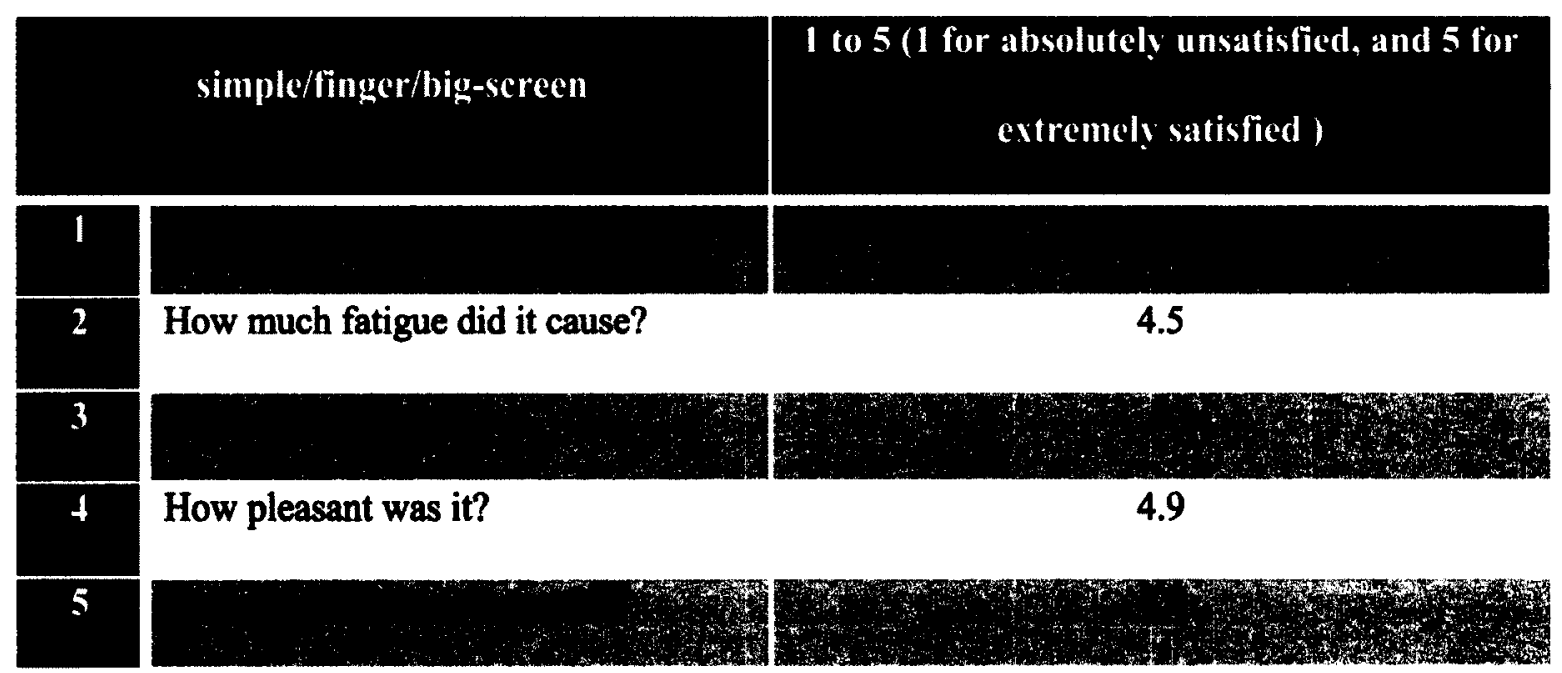


Table F.11. Questions for simple/arm/big-screen.

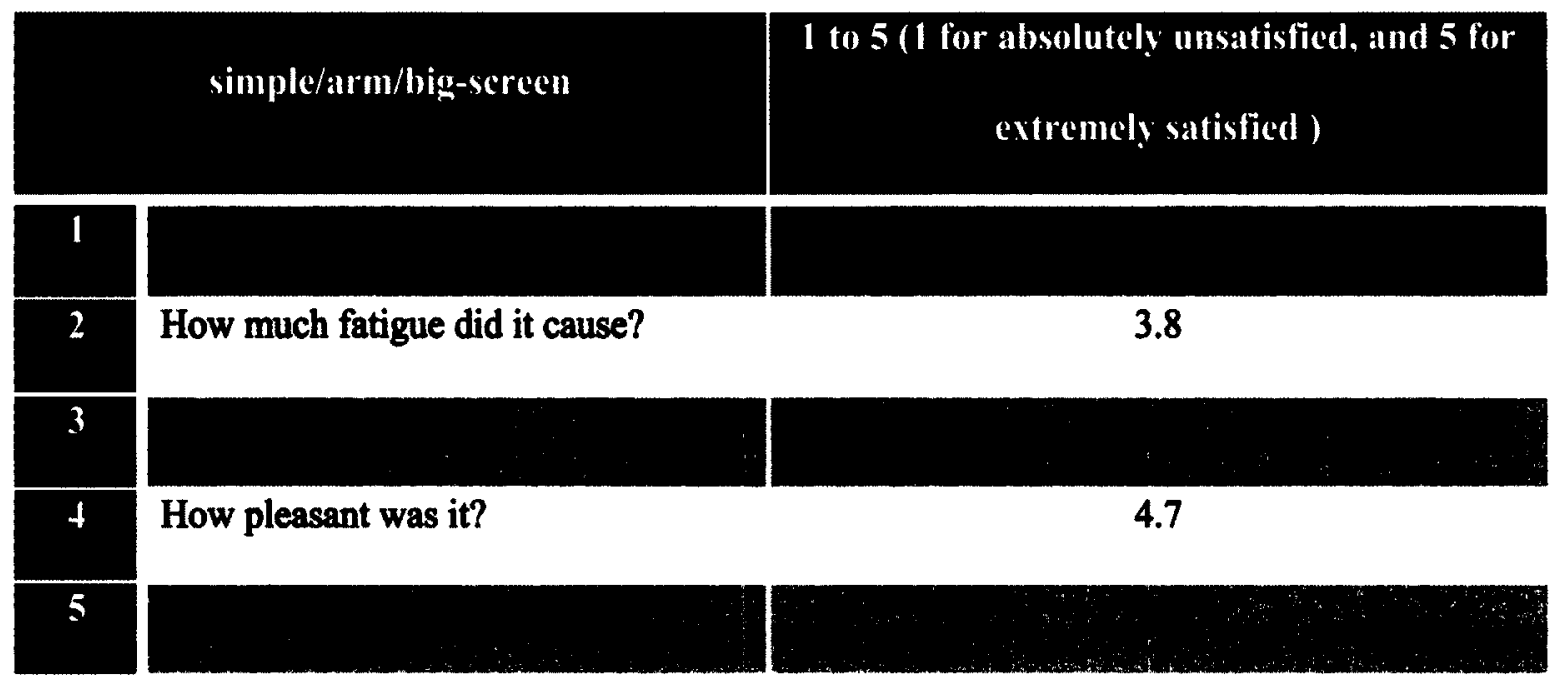

Table F.12. Questions for complex/finger/big-screen.

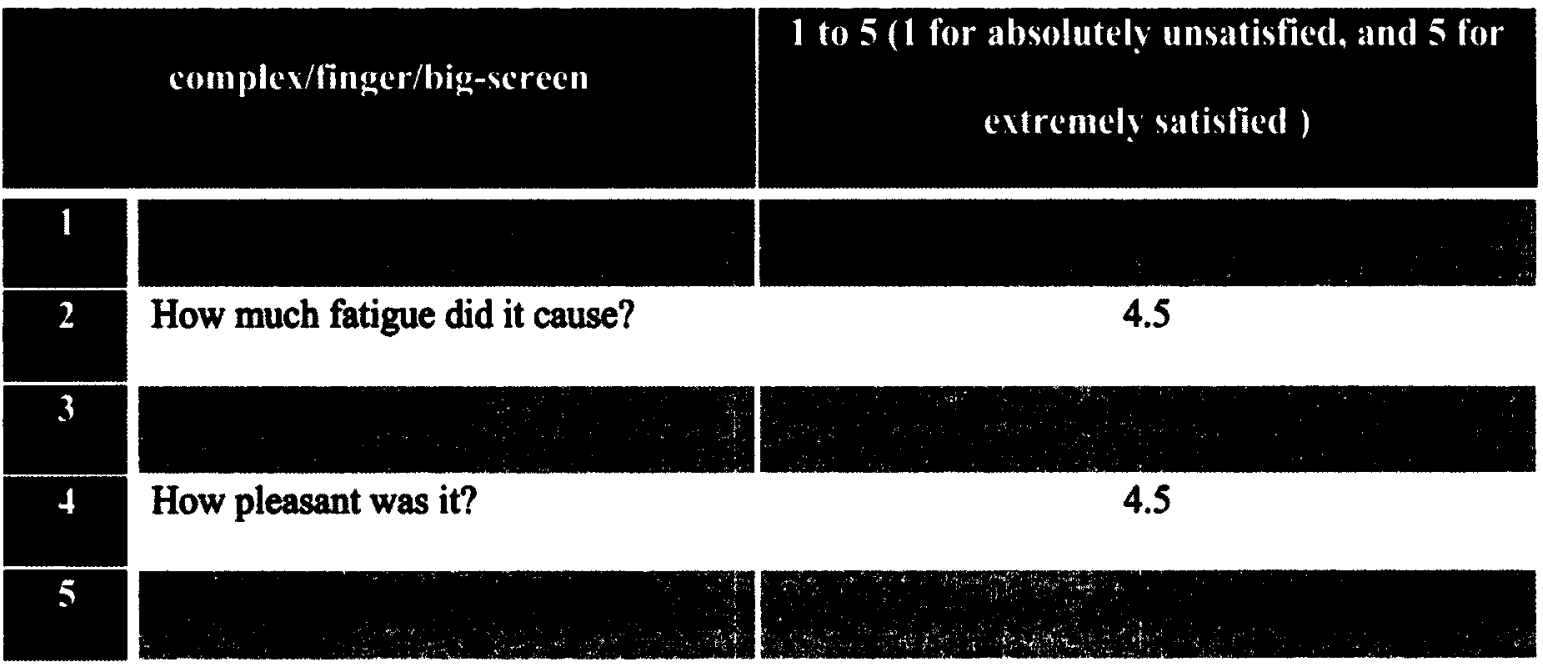


Table F.13. Questions for complex/arm/big-screen.

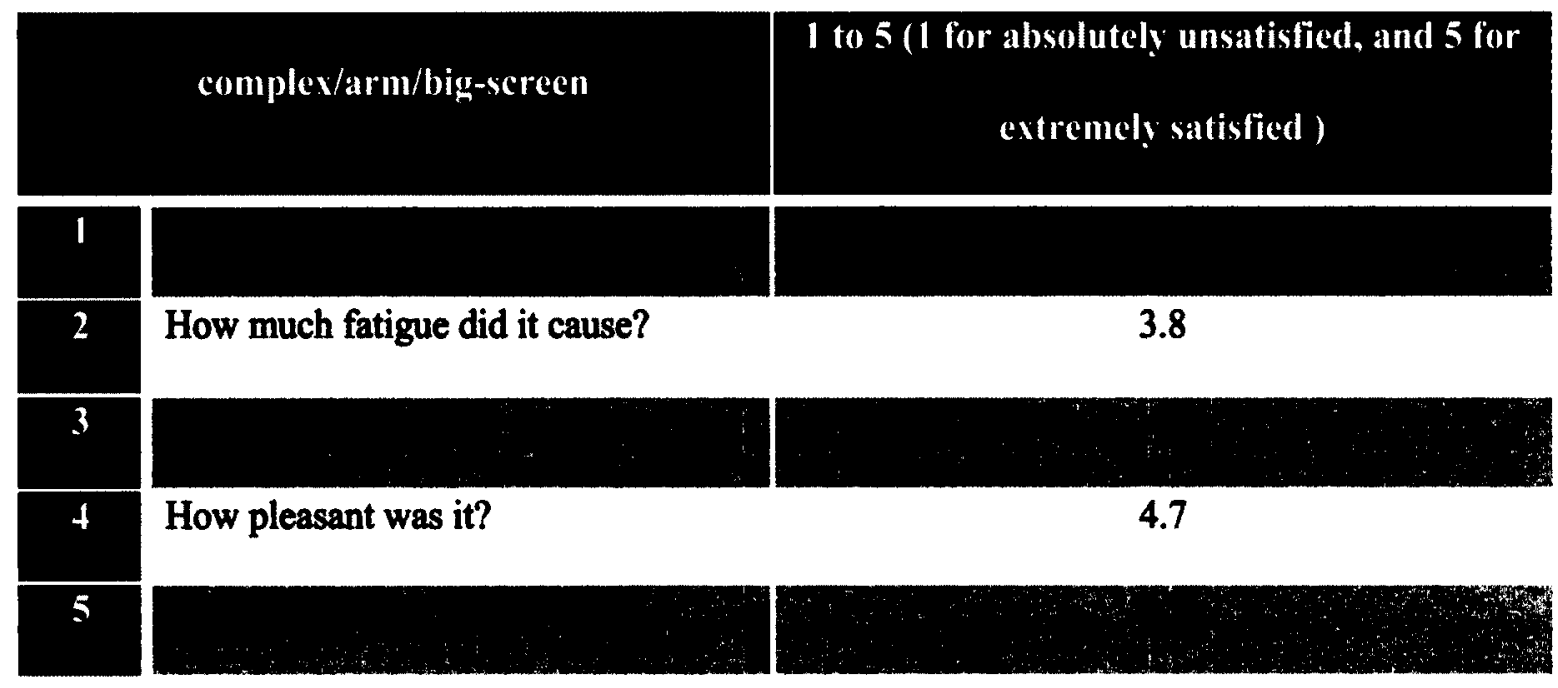

Table F.14. User satisfaction for primitive tasks.

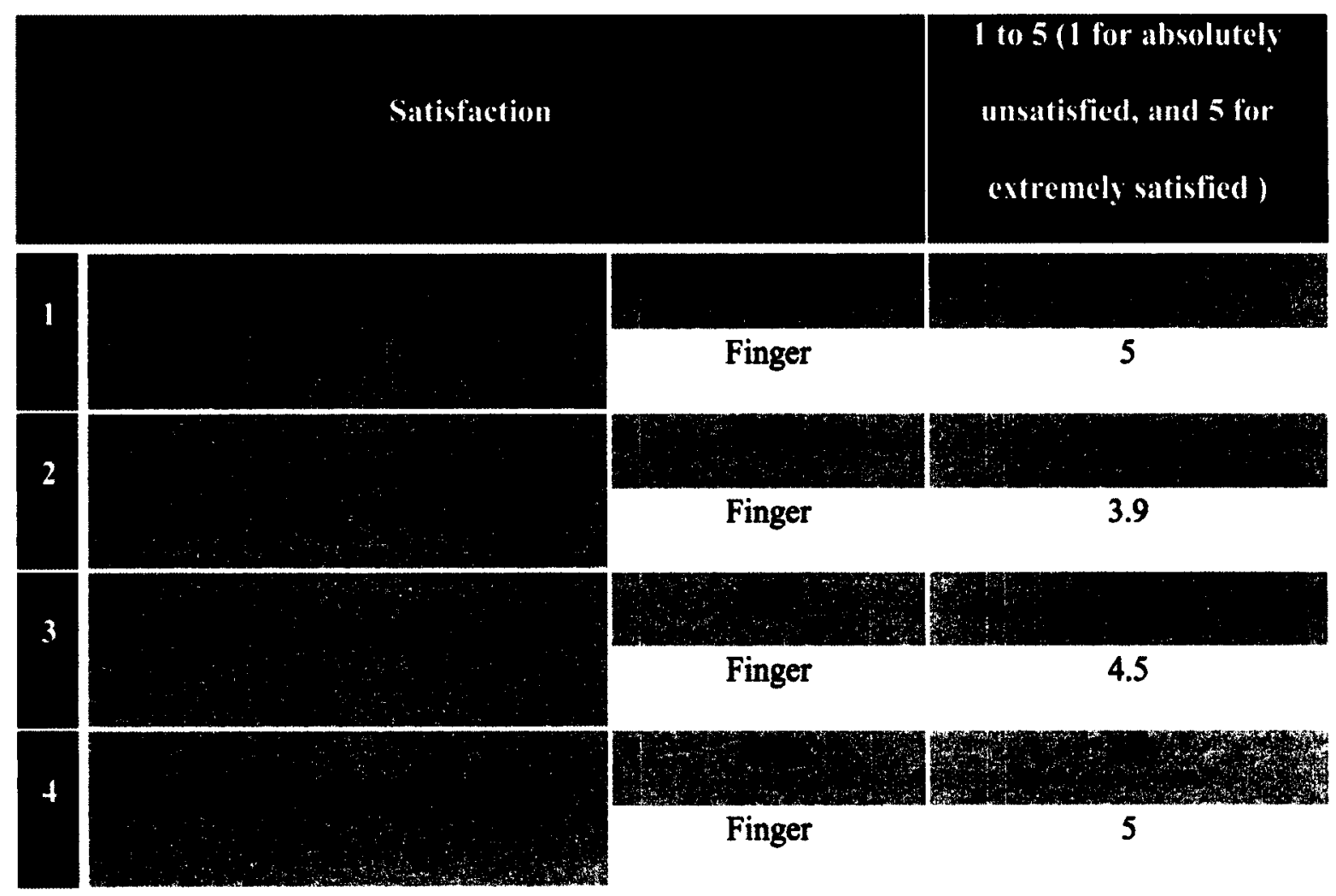




\section{References}

[1] Matthias Rehm, Nikolaus Bee and Elisabeth André, "Wave Like an Egyptian Accelerometer Based Gesture Recognition for Culture Specific Interactions," British Computer Society, 2007.

[2] Pavlovic, V., Sharma, R. \& Huang, T. (1997), "Visual interpretation of hand gestures for human-computer interaction: A review," IEEE Trans. Pattern Analysis and Machine Intelligence, 1997, Vol. 19(7), pp. $677-695$.

[3] R. Cipolla and A. Pentland, "Computer Vision for Human-Machine Interaction", Cambridge University Press, 1998, ISBN 978-0521622530.

[4] Ying Wu and Thomas S. Huang, "Vision-Based Gesture Recognition: A Review", In: Gesture-Based Communication in Human-Computer Interaction, Volume 1739 of Springer Lecture Notes in Computer Science, pages 103-115, 1999.

[5] Alejandro Jaimesa and Nicu Sebe, "Multimodal human-computer interaction: A survey, Computer Vision and Image Understanding" Vol 108, Issues 1-2, pp 116-134 Special Issue on Vision for Human-Computer Interaction, 2007.

[6] Thad Starner, Alex Pentland, "Visual Recognition of American Sign Language Using Hidden Markov Models", Massachusetts Institute of Technology.

[7] Kai Nickel, Rainer Stiefelhagen, "Visual recognition of pointing gestures for humanrobot interaction", Image and Vision Computing, Vol 25, Issue 12, 2007, pp 1875-1884.

[8] Lars Bretzner and Tony Lindeberg "Use Your Hand as a 3-D Mouse", Proc. 5th European Conference on Computer Vision (H. Burkhardt and B. Neumann, eds.), vol. 
1406 of Lecture Notes in Computer Science, (Freiburg, Germany), pp. 141--157, Springer Verlag, Berlin, June 1998.

[9] Matthew Turk and Mathias Kölsch, "Perceptual Interfaces", University of California, Santa Barbara UCSB Technical Report 2003.

[10] M Porta "Vision-based user interfaces: methods and applications," International Journal of Human-Computer Studies, 57:11, 27-73, 2002.

[11] Afshin Sepehri, Yaser Yacoob and Larry S. Davis "Employing the Hand as an Interface Device," Journal of Multimedia, Vol 1, number 2, pp 18-29.

[12] Henriksen, K. Sporring, J. and Hornbaek, K. "Virtual trackballs revisited," IEEE Transactions on Visualization and Computer Graphics, Vol. 10, Issue 2, pp. 206-216, 2004.

[13] William Freeman, Craig Weissman, "Television control by hand gestures", Mitsubishi Electric Research Lab, 1995.

[14] Do Jun-Hyeong, Jung Jin-Woo, Sung hoon Jung, Jang Hyoyoung, Bien Zeungnam, "Advanced soft remote control system using hand gesture", Mexican International Conference on Artificial Intelligence, 2006.

[15] K. Ouchi, N. Esaka, Y. Tamura, M. Hirahara, M. Doi, "Magic Wand: an intuitive gesture remote control for home appliances", International Conference on Active Media Technology, AMT'05, 2005.

[16] Lars Bretzner, Ivan Laptev, Tony Lindeberg, Sören Lenman, Yngve Sundblad "A Prototype System for Computer Vision Based Human Computer Interaction,” Technical report CVAP251, Department of Numerical Analysis and Computer Science, KTH, Royal Institute of Technology, Stockholm, Sweden, April 23-25, 2001. 
[17] Yang Liu, Yunde Jia, "A Robust Hand Tracking and Gesture Recognition Method for Wearable Visual Interfaces and Its Applications," Proceedings of the Third International Conference on Image and Graphics, ICIG'04, 2004.

[18] Kue-Bum Lee, Jung-Hyun Kim, Kwang-Seok Hong, "An Implementation of MultiModal Game Interface Based on PDAs," Fifth International Conference on Software Engineering Research, Management and Applications, 2007.

[19] Thomas Schlomer, Benjamin Poppinga, Niels Henze, Susanne Boll, "Gesture Recognition with a Wii Controller," Proceedings of the 2nd international Conference on Tangible and Embedded interaction, 2008.

[20] AiLive Inc., "LiveMove White Paper," http://www.ailive.net/, 2006.

[21] Wei $\mathrm{Du}$, Hua $\mathrm{Li}$, "Vision based gesture recognition system with single camera," Proceedings of Fifth International Conference on Signal Processing, 2000.

[22] Ivan Laptev and Tony Lindeberg "Tracking of Multi-state Hand Models Using Particle Filtering and a Hierarchy of Multi-scale Image Features," Proceedings ScaleSpace and Morphology in Computer Vision, Volume 2106 of Springer Lecture Notes in Computer Science, pages 63-74, Vancouver, BC, 1999.

[23] Christian von Hardenberg and François Bérard, "Bare-hand human-computer interaction," ACM International Conference Proceeding Series, Proceedings of the 2001 workshop on Perceptive user interfaces, Orlando, Florida, Vol. 15, pp 1-8, 2001.

[24] Lars Bretzner, Ivan Laptev, Tony Lindeberg "Hand gesture recognition using multiscale colour features, hierarchical models and particle filtering," Proceedings of the Fifth IEEE International Conference on Automatic Face and Gesture Recognition, Washington, DC, USA, pp 423-428, 2002. 
[25] Domitilla Del Vecchio, Richard M. Murray Pietro Perona, "Decomposition of human motion into dynamics-based primitives with application to drawing tasks," Automatica Vol. 39, Issue 12, pp 2085-2098, 2003.

[26] Thomas B. Moeslund and Lau Nørgaard, "A Brief Overview of Hand Gestures used in Wearable Human Computer Interfaces," Technical report: CVMT 03-02, Laboratory of Computer Vision and Media Technology, Aalborg University, Denmark.

[27] M. Kolsch and M. Turk, "Fast 2D Hand Tracking with Flocks of Features and MultiCue Integration," Proceedings of Computer Vision and Pattern Recognition Workshop, CVPRW'04, 2004.

[28] Xia Liu Fujimura, K., "Hand gesture recognition using depth data," Proceedings of the Sixth IEEE International Conference on Automatic Face and Gesture Recognition, pp 529-534, 2004.

[29] Stenger B, Thayananthan A, Torr PH, Cipolla R, "Model-based hand tracking using a hierarchical Bayesian filter," IEEE Transactions on Pattern Analysis and Machine Intelligence, 2006.

[30] A Erol, G Bebis, M Nicolescu, RD Boyle, X Twombly, "Vision-based hand pose estimation: A review", Computer Vision and Image Understanding Volume 108, Issues 1-2, October-November 2007, Pages 52-73 Special Issue on Vision for Human-Computer Interaction.

[31] D. Tzovaras, "Multimodal User Interfaces: From Signals to Interaction," Springer, Heidelberg, 2008. 
[32] Liu Yun; Zhang Peng; , "An Automatic Hand Gesture Recognition System Based on Viola-Jones Method and SVMs," Computer Science and Engineering, 2009. WCSE' '09. Second International Workshop on, vol.2, no., pp.72-76, 28-30 Oct. 2009.

[33] P. Kortum, "HCI Beyond the GUI: Design for Haptic, Speech, Olfactory, and Other Nontraditional Interfaces," Morgan Kaufmann Publishers, 2008, pp. 75-106.

[34] J. Weissmann and R. Salomon, "Gesture Recognition for Virtual Reality Applications Using Data Gloves and Neural Networks," IJCNN 99 International Conference On Neural Networks, Vol 3. 1999, pp. 2043-2046.

[35] T. G. Zimmerman, J. Lanier, C. Blanchard, S. Bryson, and Y. Harvill, "A Hand Gesture Interface Device," CHI+GI, 1987, pp. 189-192.

[36] T. B. Moeslund and L. Norgaard, "A brief overview of hand gestures used in wearable human computer interfaces," Technical report, Aalborg University, Denmark, 2002.

[37] P. Viola and M. Jones, "Robust Real-time Object Detection," 2nd International Workshop on Statistical and Computerational Theories of Vision, July 2001.

[38] P. Viola and M. Jones, "Rapid Object Detection Using a Boosted Cascade of Simple Feature," IEEE Computer Vision and Pattern Recognition, Vol 1, Dec, 2001, pp. 551518.

[39] Q. Chen, M. D. Cordea, E. M. Petriu, A. R. Varkonyi- Koczy, and T. E. Whalen, "Human-Computer Interaction for Smart Environment Applications Using Hand-Gesture and Facial-Expressions," International Journal of Advanced Media and Communication, vol. 3 n.1/2, June 2009, pp. 95-109. 
[40] Kolsch and M. Turk, "Robust Hand Detection," In International Conference on Automatic Face and Gesture Recognition, 2004.

[41] M. Kolsch and M. Turk, "Anaysis of Rotational Robustness of Hand Detection with a Viola-Jones Detector," In IAPR International Conference of Pattern Recognition, 2004.

[42] Q. Zhang, F. Chen, and X. Liu, "Hand Gesture Detection and Segmentation Based on Difference Background Image with Complex Background," The 2008 International Conference on Embedded Software and Systems, ICESS' 08, 2008, pp. 338- 343.

[43] L. Anton-Canalis, E. Sanchez-Nielsen, and M. Castrillon- Santana, "Hand Pose Detection for Vision-based Gesture Interfaces. Conference on Machine Vision Applications," Tsukuba Science City, Japan, May 16-18, 2005.

[44] S. Marcel, O. Bernier, J. E. Viallet, and D. Collobert, "Hand Gesture Recognition using Input-Output Hidden Markov Models," Proc. of the FG'2000 Conference on Automatic Face and Gesture Recognition, 2000.

[45] F. Chen, C. Fu, and C. Huang, "Hand gesture recognition using a real-time tracking method and hidden Markov models," Image and Vision Computing, 2003, pp. 745-758.

[46] S. C. Ahn, T. S. Lee, I. J. Kim, Y. M. Kwon, and H. G. Kim, "Computer VisionBased Interactive Presentation System," Proceedings of Asian Conference for Computer Vision 2004, January, 2004.

[47] G. Jain, "Vision-Based Hand Gesture Pose Estimation for Mobile Devices," University of Toronto, 2009.

[48] O. Aran, I. Ari, F. Benoit, A. Campr, A.H. Carrillo, P. Fanard, L. Akarun, A. Caplier, M. Rombaut, and B. Sankur, "Sign Language Tutoring Tool," eNTERFACE 2006, The Summer Workshop on Multimodal Interfaces, Croatia, 2006. 
[49] Bhuyan, M.K.; Ghosh, D.; Bora, P.K.; "Co-articulation Detection in Hand Gestures," TENCON 20052005 IEEE Region 10, vol., no., pp.1-4, $21-24$ Nov. 2005.

[50] Yun Liu; Peng Zhang; "Vision-Based Human-Computer System Using Hand Gestures," Computational Intelligence and Security, 2009. CIS '09. International Conference on , vol.2, no., pp.529-532, 11-14 Dec. 2009.

[51] Raheja, J.L.; Shyam, R.; Kumar, U.; Prasad, P.B.; , "Real-Time Robotic Hand Control Using Hand Gestures," Machine Learning and Computing (ICMLC), 2010 Second International Conference on , vol., no., pp.12-16, 9-11 Feb. 2010.

[52] Pang, Yee Yong; Ismail, Nor Azman; Gilbert, Phuah Leong Siang; , "A Real Time Vision-Based Hand Gesture Interaction," Mathematical/Analytical Modelling and Computer Simulation (AMS), 2010 Fourth Asia International Conference on, vol., no., pp.237-242, 26-28 May 2010.

[53] Chenglong Yu; Xuan Wang; Hejiao Huang; Jianping Shen; Kun Wu; , "VisionBased Hand Gesture Recognition Using Combinational Features," Intelligent Information Hiding and Multimedia Signal Processing (IIH-MSP), 2010 Sixth International Conference on , vol., no., pp.543-546, 15-17 Oct. 2010.

[54] El-Bendary, N.; Zawbaa, H.M.; Daoud, M.S.; Hassanien, A.E.; Nakamatsu, K.; "ArSLAT: Arabic Sign Language Alphabets Translator," Computer Information Systems and Industrial Management Applications (CISIM), 2010 International Conference on , vol., no., pp.590-595, 8-10 Oct. 2010.

[55] R. Harper, T. Rodden, Y. Rogers and A. Sellen, "Being Human: Human-Computer Interaction in the year 2020," Microsoft Corporation, 2008.

[56] "Kinect." Wikipedia. Sep. 2011. Oct. 2011. <http://en.wikipedia.org/wiki/Kinect>. 
[57] "Programmer Guide." Documentation. 2010. 21 Jan. 2010. <www.OpenNI.org>.

[58] "Prime Sensor ${ }^{\text {TM }}$ NITE 1.3 Framework Programmer's Guide." PrimeSense. 2010. 19 Apr. 2011. < http://pr.cs.cornell.edu/humanactivities/data/NITE.pdf >.

[59] Dimitrov, Smilen. "HCI Challenges." 2010. 7 August 2011. $<$ www.smilen.net/st/files/st_intro_01.ppt>.

[60] "Motion Gestures." Apple Computer Inc. 2005. 17 Sept. 2011. $<$ http://manuals.info.apple.com/en/motion_2_gestures_reference.pdf $>$.

[61] royshilk. "Opencv 2d hand pose-estimator." 23 Dec. 2010. 21 Apr. 2011. $<$ http://www.youtube.com/watch?v=uETHJQhK14>.

[62] "Prime Sensor ${ }^{\text {TM }}$ NITE 1.3 Framework Programmer's Guide." PrimeSense. 2010. 19 Apr. 2011. < http://pr.cs.cornell.edu/humanactivities/data/NITE.pdf $>$.

[63] "OpenCV." OpenCVWiki. 24 August 2011. 2 September 2011. $<$ http://opencv.willowgarage.com/wiki/>.

[64] "Microsoft Kinect SDK vs. PrimeSense OpenNI." Brekel. July 2011. 15 Aug. 2011. $<$ http://www.brekel.com/?page_id=671>.

[65] "Introducing Kinect for Xbox 360." Microsoft Corporation. 2011. $<$ http://www.xbox.com/en-CA/Kinect>.

[66] "The 3D Tech Behind Virtual Production using Kinect." Autodesk. 12 Aug. 2011. 2 Sept. 2011 . <http://www.youtube.com/watch?v=fZCJnHk9qm4>.

[67] Hyong Su Kim, "Gesture Definition Approaches and Limitations", Vancouver, BC, Canada, CHI, 2011. 
[68] 3D Remote Interface for Smart Displays, Vancouver, BC, Canada, CHI, 2011.

[69] Marcio C. Cabral, Carlos H. Morimoto, Marcelo K. Zuffo, "On the usability of gesture interfaces in virtual reality environments", CLIHC'05, 2005, Cuernavaca, México, 2005.

[70] Norman Villaroman, Dale Rowe, Bret Swan, “Teaching Natural User Interaction Using OpenNI and the Microsoft Kinect Sensor", SIGITE, West Point, New York, USA, 2011.

[71] Gilles Bailly, Robert Walter1, Jörg Müller, Tongyan Ning, and Eric Lecolinet, "Comparing Free Hand Menu Techniques for Distant Displays Using Linear, Marking and Finger-Count Menus" IFIP, 2011.

[72] Jong-wook Kang, Dong-jun Seo, and Dong-seok Jung, "A Study on the control Method of 3-Dimensional Space Application using KINECT System", IJCSNS International Journal of Computer Science and Network Security, VOL.11 No.9, September 2011.

[73] Andrew Bragdon, Rob DeLine, Ken Hinckley, Meredith Ringel Morris, "Code Space: Touch + Air Gesture Hybrid Interactions for Supporting Developer Meetings", ITS, Kobe, Japan, 2011.

[74] Moniruzzaman Bhuiyan, Rich Picking, "A Gesture Controlled User Interface for Inclusive Design and Evaluative Study of Its Usability", Journal of Software Engineering and Applications, 2011.

[75] Lars C. Ebert, Gary Hatch, Garyfalia Ampanozi, Michael J. Thali, and Steffen Ross, "You Can't Touch This: Touch-free Navigation Through Radiological Images", SAGE, 2011. 
[76] Stefan Greuter, David J Roberts, "Controlling Viewpoint from Markerless Head Tracking in an Immersive Ball Game Using a Commodity Depth Based Camera", IEEE DS-RT, 2011.

[77] "Innovation Days." Digifest. September 2011. 30 October 2011. $<$ http://torontodigifest.ca/2011/innovation-days/\#arya>.

[78] "Two Carleton Grad Students Open Digital Gate to Virtual Worlds." Carleton University/ Graduate Admissions/ News. 3 Nov. 2011. 17 Nov. 2011. $<$ http://wwwl.carleton.ca/graduate/2011/two-carleton-grad-students-open-digital-gate-to-virtualworlds $>$.

[79] "PrimeSense Supplies 3-D-Sensing Technology to "Project Natal" for Xbox 360." Microsoft News Centre. 31 March 2010. 22 December 2011. $<$ http://www.microsoft.com/Presspass/press/2010/mar10/03-31PrimeSensePR.mspx>.

[80] "Gesture Recognition and Computer Vision Control Technology." GestureTek. December 2011. <http://www.gesturetek.com >.

[81] "WIMP (Computing)." Wikipedia Encyclopedia. December 2011. $<$ http://en.wikipedia.org/wiki/WIMP_(computing) >.

[82] Boussemart, Y., Rioux, F., Rudzicz, F., Wozniewski, M. \& Cooperstock, J. R. “A framework for $3 \mathrm{~d}$ visualisation and manipulation in an immersive space using an untethered bimanual gestural interface." In: VRST '04: Proceedings of the ACM symposium on Virtual reality software and technology. ACM Press, New York, NY, USA, pp. 162-165, 2004.

[83] Emanuele Trucco, Alessandro Verri, "Introductory Techniques for 3-D Computer Vision", Prentice Hall, 1998. 